\title{
Quantum-classical modeling of non-adiabatic transitions in polyatomic systems
}

\author{
Dissertation \\ zur Erlangung des Doktorgrades \\ der Mathematisch-Naturwissenschaftlichen Fakultäten \\ der Georg-August-Universität Göttingen
}

vorgelegt von

Diana Constanta Tranca

aus Romania

Göttingen, 2009 
D7

Referentin/Referent: Prof. Dr. Jürgen Troe

Koreferentin/Koreferent: Prof. Dr. Jörg Schroeder

Tag der mündlichen Prüfung: 30.10.2009 


\section{Contents}

$\begin{array}{lll}1 & \text { INTRODUCTION } & 7\end{array}$

2 SCOPE OF THE THESIS 11

3 QUANTUM-CLASSICAL APPROACHES TO THE NON-ADIABATIC DYNAM$\begin{array}{ll}\text { ICS } & 15\end{array}$

3.1 Transition State Theory . . . . . . . . . . . . . . . . . . 16

3.2 Molecular Dynamics with Quantum Transitions . . . . . . . . . . . . . 18

3.2.1 Mean Field (Ehrenfest) Approximation (MFA) . . . . . . . . . . . . . 19

3.2.2 Surface Hopping $(\mathrm{SH}) \ldots \ldots \ldots \ldots$

3.3 Reduced Density Matrix Methods _. . . . . . . . . . . . . . . . . . 21

3.3.1 Quantum-Classical Liouville Equation . . . . . . . . . . . . . . . . 21

3.3.2 Redfield Theory . . . . . . . . . . . . . . . . . . . 24

3.4 Non-Markovian Quantum-Classical Approximation . . . . . . . . . . . . . 27

4 STATISTICAL APPROACH TO NON-ADIABATIC TRANSITIONS 29

5 HOT ABSORPTION SPECTRA OF POLYATOMIC MOLECULES 33

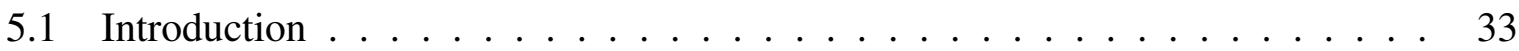

5.2 NQCA and the lineshape of the absorption spectrum $\ldots \ldots \ldots$

5.3 Model and simulation procedure . . . . . . . . . . . . . . . . . 39 
5.4 Calculation of multi-dimensional trajectories $\ldots \ldots \ldots$. . . . . . . 40

5.5 Energy calculations for trans-stilbene and coumaric acid . . . . . . . . . . 42

5.6 Results - Solvent influence on the transition energy gap . . . . . . . . . . . . 47

5.7 Results - Hot absorption spectra simulations by NQCA . . . . . . . . . . . 51

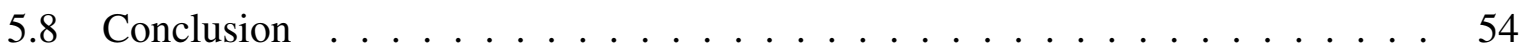

6 NONADIABATIC PHOTOISOMERIZATION OF POLYATOMIC MOLECULES 57

6.1 Introduction . . . . . . . . . . . . . . . . . 57

6.2 Basic assumptions of the model . . . . . . . . . . . . . . . 59

6.3 Photoisomerization processes of polyatomic molecules . . . . . . . . . . . 64

6.4 Photoisomerization kinetics of trans-stilbene molecule . . . . . . . . . . 66

6.5 Photoisomerization kinetics of p-coumaric acid molecule . . . . . . . . . 70

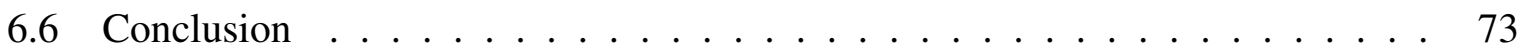

A APPENDIX: Input description GAMESS US software 75

B ENERGY CALCULATIONS OF GROUND STATE FOR COUMARIC ACID 79

B.1 Optimize the ground state for p-coumaric acid . . . . . . . . . . . 80

B.2 Hessian calculations for the ground state $\ldots \ldots \ldots \ldots 2$

B.3 Energy calculations for ground state . . . . . . . . . . . . . . . 84

C ENERGY CALCULATIONS OF EXCITED STATE FOR COUMARIC ACID 87

C.1 Optimize the second excited state for p-coumaric acid . . . . . . . . . . 88

C.2 Hessian calculations for the excited state . . . . . . . . . . . . 90

C.3 Energy calculation for excited state . . . . . . . . . . . . . . . . 92

D APPENDIX: Transition dipole moment calculations 95

D.1 Equilibrium ground state for benzene molecule . . . . . . . . . . . . . 96

D.2 Energy calculations for ground state - benzene molecule . . . . . . . . . . 98 
D.3 Transitions dipole moment calculations for ground state . . . . . . . . . . . 100

E APPENDIX: Non-adiabatic coupling vector calculations

E.1 Non-adiabatic couplings vectors for coumaric acid molecule . . . . . . . . 105

E.2 Non-adiabatic couplings vectors for stilbene molecule . . . . . . . . . . . 106 


\section{1}

\section{INTRODUCTION}

Non-adiabatic transitions ${ }^{1,2}$ play an important role in the reaction dynamics of many physical, chemical, and biological systems. Very often the regions, where the adiabatic BornOppenheimer approximation ${ }^{3,4}(\mathrm{BO})$ breaks down, provide favorable conditions for the transitions between the corresponding quantum states, and the non-adiabatic reaction pathway may become the dominant one.

Theoretical description of non-adiabatic transitions has been the subject of extensive investigations, ranging from one-dimensional models to realistic polyatomic systems. The analytic solution $^{5,6}$ obtained by Landau and Zener (LZ) for the transition probability between linear crossing terms for single passage of the intersection point with constant velocity, has provided deep insight into the physics of the process, the dependence on characteristic parameters of the model, and introduced a classification of the adiabatic and the non-adiabatic regimes. The LZ model was subsequently extended to overcome its limitations ${ }^{1,2}$ by taking into account tunneling effects, multiple passages through the non-adiabaticity region, influence of dissipative environments, ${ }^{7}$ etc.

However, results obtained for non-adiabatic transitions in model systems can hardly be applied directly to realistic polyatomic molecules where reaction coordinates are often multidi- 
mensional, and the reaction dynamics often exhibits quantum coherence and memory effects, ${ }^{8}$ so that the description of non-adiabatic dynamics in terms of transition probabilities may be inadequate. Besides, calculations of the transition probabilities are insufficient for theoretical modeling of light absorption/emission spectra, which are directly related to the time evolution of the quantum phase of the transitions. ${ }^{9-12}$

These problems have stimulated the development of alternative treatments of non-adiabatic dynamics, based on the ideas of the Quantum-Classical Approximation (QCA), where only important degrees of freedom are treated quantum-mechanically, and the remaining ones by means of classical mechanics. The QCA combines linear scaling, an increase in the number of degrees of freedom, which is of primary importance when considering polyatomic molecules, and a proper treatment of non-adiabatic transitions, which are inherently quantum-mechanical processes.

Nonetheless, the interface between the deterministic equations of motion of classical mechanics and the intrinsically probabilistic description of quantum mechanics is a challenging problem which is not completely solved yet. In the most straightforward attempts one adds quantum transitions directly to the classical trajectories, ${ }^{13-15}$ employing methods called Molecular Dynamics with Quantum Transitions, briefly MDQT. However the intuitive construction of MDQT approaches has encountered severe intrinsic problems, ${ }^{16-18}$ which cannot be systematically resolved because of a lack of consistent derivation.

Recently, an alternative Non-Markovian Quantum-Classical Approximation ${ }^{19-22}$ (NQCA) has been suggested, which has a wide and well established applicability range. It is free of drawbacks inherent in MDQT methods, and is capable of dynamical treatment of quantum transitions with a full account of detailed balance, quantum coherence and memory effects. NQCA can easily be combined with ab initio Molecular Dynamics (MD) and quantum chemical methods to describe the evolution of the classical degrees of freedom and the quantum part of the problem, respectively, and often has a computational effectiveness at least comparable with 
those of MDQT. In this thesis we consider a wide class of reacting systems where the time scale required for equilibration in the phase space of the potential energy surfaces (PES) is short compared to the time scale of the transitions between them. Such a situation seems to be rather typical for non-adiabatic transitions between different electronic states in polyatomic molecules, where a high density of vibronic states facilitates Intramolecular Vibrational Energy Redistribution (IVR), thus providing an efficient relaxation of the phase space distribution.

The structure of the thesis is as follows. In Chapter (2) we outline the scope of the thesis. The existing quantum-classical approaches and their applications are reviewed in Chapter (3). The kinetic equations of the quantum-classical approach used for the ab initio simulations of the non-adiabatic dynamics are presented in Chapter (4). The application of the approach to the $a b$ initio modeling of hot absorption spectra of polyatomic molecules is described in Chapters (5). Then, in the Chapters (6) we apply the approach to estimate the efficiency of the non-adiabatic pathway of the trans-cis photoisomerization of stilbene and p-coumaric acid. In the Appendices $(\mathrm{A}, \mathrm{B}, \mathrm{C}, \mathrm{D}, \mathrm{E})$ a more detailed description of the computational details of the energy and structure parameters calculations of the ground and excited states are presented, as well as the results of the transition dipole moment and non-adiabatic coupling vectors calculations. 


\section{SCOPE OF THE THESIS}

The basic subject of this thesis is the investigation of non-adiabatic dynamics in polyatomic molecules by using the recently proposed method - (the Non-Markovian Quantum Classical Approximation (NQCA)). ${ }^{19-22}$

The non-adiabatic dynamics of small systems with a few degrees of freedom is well investigated by now using both fully quantum mechanical treatments and approximate approaches ranging from rigorous semiclassical ones to various hybrid schemes like Surface Hopping (SH), Mean-Field Approximation (MFA). However, realistic reactions involve polyatomic molecules, where the reaction coordinates are often multidimensional, which restricts the range of applicable methods to the quantum-classical approaches.

The majority of the existing quantum-classical methods can hardly be applied to the ab initio modeling of the non-adiabatic reactions. They either have severe intrinsic problems $(\mathrm{SH}$, MFA, Quantum-Classical Liouville Equation), or too narrow applicability range (Redfield Theory), see Chapter (3) for more detailed discussion. In contrast, the recently developed NQCA approach is free from the drawbacks inherent in MDQT, and is capable of dynamic treatment of quantum transitions with full account of the detailed balance, quantum coherence and memory

effects. ${ }^{19-22}$ It is based on a classical mechanics description of the dynamics on the PES with 
a quantum mechanical treatment of transitions between different PESs, and assumes fast IVR and equilibration in the phase space of the PESs to the temperature of the surrounding media. The key difference of the NQCA from the other quantum-classical methods is the consistent and accurate treatment of the quantum transition phase, which is of fundamental importance for any ab initio approach to the quantum transitions.

In the present thesis the NQCA method is applied to the investigation of the Ultraviolet/Visible (UV/vis) absorption spectra and of the photoisomerization processes of polyatomic molecules. For both cases the corresponding computer programs have been developed. The programs combine the quantum-chemical calculations of the PESs and the MD simulations of the dynamics in their phase spaces. All the input parameters were estimated either from the high-level quantum-chemical calculations (equilibrium geometries, normal modes and their frequencies, non-adiabatic coupling vectors), or from the spectroscopical data.

First, we will adopt and use the NQCA method for the simulation of the UV/vis absorption spectra of polyatomic molecules, e.g. benzene and trans-stilbene. The corresponding spectra look different, the spectra of benzene exhibit vibronic structure, whereas those of transstilbene is broad and nearly structureless. The absorption spectra are directly related to the evolution of the quantum phase of transitions between the electron PESs, which allows to estimate the applicability and accuracy of the NQCA for the modeling of non-adiabatic reactions in polyatomic systems. Ab initio simulations of the hot absorption spectra in the gas phase were carried out and directly compared to the available experimental data. A good agreement between the theoretically modeled and the experimentally measured spectra demonstrates the consistency of NQCA, and opens broad perspectives to the application of the recently developed quantum-classical approximation to the ab initio modeling of non-adiabatic reactions in complex systems.

Subsequently, the NQCA method will be used for the simulation of the non-adiabatic pathway of the trans-cis photoisomerization of stilbene and p-coumaric acid. These two molecules 
look similar, but photoisomerization of p-coumaric acid is much faster and the proper theoretical description have to reproduce such a difference in the time scales of the reaction.

Actually, there are exist two alternative models of the trans-cis photoisomerization of stilbene and similar molecules. In an adiabatic model one assumes the existence of a small barrier on the first excited state $\left(S_{1}\right)$ and a fast IVR, so that the RRKM model can be used to calculate the reaction rate. The adiabatic model was successfully applied to the description of molecular beam experiments and an effective barrier height was estimated to be about $1200 \mathrm{~cm}^{-1}$. The activated barrier crossing is assumed to be the rate limiting step, and a radiationless transition into the ground state takes place from the twisted conformation, which belongs to distinct electron PES. ${ }^{23-25}$

Alternatively, a non-adiabatic model, which accounts for the transitions to the other excited state prior to the isomerization, was suggested to explain the photoisomerization mechanism. It is supported by the fact that the twisted conformation of stilbene, where the radiationless transition to the ground state takes place, belongs to the distinct electronic state $\left(S_{2}\right)$ characterized as the double excited or zwitterionic state. ${ }^{26-29}$

We employ the non-adiabatic model of the photoisomerization, and assume that the transition $S_{1}$ to $S_{2}$ state takes place near the planar geometry of trans-stilbene, which is the rate limiting step. The subsequent twist around the central bond proceeds along $S_{2}$ PES, where it is nearly barrierless. The time scales of the photoisomerization kinetics were well reproduced. For gas-phase stilbene at room temperature the non-adiabatic pathway gives a characteristic time of approximately $630 \mathrm{ps}$, whereas p-coumaric acid photoisomerization under the same conditions is predicted to be faster than $1 \mathrm{ps}$. A deep insight into the mechanism of the reaction reveals, that in the case of stilbene the non-adiabatic conversion between the single and the double excited states proceeds without surface crossing events, via a relaxation-like mechanism, when the intrinsic modes of the molecule play the role of a heat reservoir. On the other hand, the relative energetics of the single and the double excited states in p-coumaric acid is the opposite, which 
leads to the frequent surface crossing events on the time scale of the reaction and, as the result, to a much faster photoisomerization. 


\section{3}

\section{QUANTUM-CLASSICAL APPROACHES}

\section{TO THE NON-ADIABATIC DYNAMICS}

This chapter will give an overview of state of the art methods one can use in order to study the non-adiabatic dynamics of the polyatomic molecules. The breakdown of the adiabatic BornOppenheimer approximation usually takes place when the energy splitting between the adiabatic potential energy surfaces, obtained at the fixed positions of the atomic nuclei, becomes comparable to the non-adiabatic coupling terms, so that it can no longer be taken into consideration by means of perturbation theory. These regions of the non-adiabaticity are typically characterized by a high efficiency of the transitions between the quantum states involved.

The non-adiabatic dynamics of small systems (i.e. with a few degrees of freedom) is well investigated by now using both fully quantum mechanical treatments ${ }^{30}$ and approximate approaches ranging from the rigorous semiclassical ${ }^{13-15,30}$ ones to the various hybrid schemes adding quantum transitions to classical trajectories, e.g. Surface Hopping, ${ }^{13}, 14$ and Mean-Field Approximation. ${ }^{13,14}$ However, realistic reaction complexes involve polyatomic molecules, and, therefore, the reaction coordinates are often multi-dimensional, which restricts the range of applicable methods to quantum-classical approaches. ${ }^{13,14,19,20,22,31}$ These methods use classical 
mechanics to describe the dynamics of nucleus on the given PES, while keeping a quantummechanical treatment of the transitions between them. These methods have linear scaling when increasing the number of the degrees of freedom and can be applied to study non-adiabatic dynamics of polyatomic reacting systems.

A detailed description of all the existing quantum-classical methods goes beyond the scope of this work, therefore we give a short summary with a main focus on the advantages and disadvantages of each approach.

\subsection{Transition State Theory}

Transition State Theory, developed in the 1930 s by Wigner ${ }^{32}$ and others, ${ }^{33}$ plays a central role in the theory of chemical reaction rates in condensed and gas phase. It assumes the existence of a hypersurface in phase space (transition state), dividing the phase space of reactants and products. The rate constant of the adiabatic reaction (proceeding along the same potential energy surface) in the framework of TST is defined as the equilibrium flux through the transition state, and can be written as

$$
k_{\mathrm{TST}}^{\mathrm{a}}=\frac{k_{B} T}{h} \frac{Q^{\ddagger}}{Q_{R}} \exp \left(-\frac{E_{0}}{k_{B} T}\right)
$$

where $Q^{\ddagger}$ is the partition function of the transition state (it involves all degrees of freedom except the reaction coordinate), $Q_{R}$ is the partition function of the reactants, the energy $E_{0}$ is the difference in zero point energy between the transition state and the reactants, $T$ is the temperature, whereas $h$ and $k_{b}$ are the Planck and the Boltzmann constant, respectively.

Similar ideas were used subsequently to extend the TST to the non-adiabatic reactions, when the reactants and the products belong to distinct PESs. In this case the transition state is associated with the surface crossing region, and the reaction rate becomes ${ }^{34,35}$

$$
k_{\mathrm{TST}}^{\mathrm{na}}=\frac{Q_{R}^{\ddagger}}{Q_{R}} \exp \left(-\frac{E_{0}}{k_{B} T}\right) \theta(T)
$$


where $Q_{R}^{\ddagger}$ is the partition function of the reactants at the surface crossing, and $\theta(T)$ accounts for the (usually small) transition probability $P(v)$ for a single passage through the intersection point. The latter depends on the velocity $v$ along the reaction coordinate and has to be averaged over the Maxwellian velocity distribution, which yields

$$
\theta(T)=\frac{1}{h} \int_{0}^{\infty} P(v) v \exp \left(\frac{-v^{2}}{2 k_{B} T}\right) d v .
$$

Note, that $P(v)$ is an external parameter in non-adiabatic TST. Several theoretical approaches can be used to estimate this quantity under various conditions, see Ref. ${ }^{1}$ and references therein for more details.

However, the separation of adiabatic dynamics and non-adiabatic transitions is not well justified for reactions in condensed phase. First, the concept of a single-passage transition probability assumes that the velocity autocorrelation decays slowly in the region of the nonadiabaticity, otherwise the overall transition probability cannot be represented as the sum of the single-passage event contributions. It is well known, that the surrounding media may strongly affect the motion of the internal degrees of freedom, especially of the low-frequency ones, and change it from a dynamical to a diffusion-like process. It also influences the velocity autocorrelation, which becomes zero in the limiting case of continuous diffusion. Second, the non-adiabaticity region can be broad itself, and involve multiple surface crossings. Third, the reaction coordinate can be multidimensional. Last but not least, quantum interference effects can directly manifest themselves in the reaction dynamics, such that the description of nonadiabatic processes in terms of transition probabilities may not be adequate.

A joint consideration of reactant mobility and non-adiabatic transitions requires $a b$ initio methods with a dynamical treatment of the quantum phase. Fully quantum-mechanical and semiclassical approaches to realistic multidimensional systems are not feasible in view of the unfavorable exponential scaling with increasing the number of degrees of freedom. On the other hand, quantum-classical approaches, when only a few important degrees of freedom are treated quantum-mechanically, whereas the other degrees of freedom are treated by means of classi- 
cal mechanics, are able to provide a method with a favorable scaling and accurate quantummechanical consideration of the subsystem of interest. Nevertheless, despite the fact that the basic idea of the quantum-classical approach is rather simple, the existing approaches are very different and not equivalent to each other. We briefly review and discuss them below.

\subsection{Molecular Dynamics with Quantum Transitions}

In the most straightforward attempts to construct a quantum-classical approach one adds quantum transitions directly to the classical trajectories. Different methods are used to describe the dynamics of the system, including non-adiabatic transitions, for example the Mean Field Approximation $^{36-39}$ (MFA), and the Surface Hopping ${ }^{38,40-42}(\mathrm{SH})$. Both approaches describe the state of the quantum subsystem by the wavefunction $|\Psi(\mathbf{r} ; t)\rangle$, which obeys the time-dependent Schrödinger equation

$$
i \hbar \frac{d}{d t}|\Psi(\mathbf{r} ; t)\rangle=\hat{H}(\mathbf{r}, \mathbf{R}(t))|\Psi(\mathbf{r} ; \mathbf{t})\rangle
$$

where $\mathbf{r}$ and $\mathbf{R}$ are the quantum and the classical coordinates, correspondingly, $\hat{H}(\mathbf{r}, \mathbf{R}(t))$ is the Hamiltonian of the quantum subsystem, which parametrically depends on the coordinates of the classical subsystem. The explicit time-dependence of the Hamiltonian in Eq. (3.4) in accordance with the given trajectory $\mathbf{R}(t)$ induces transitions between its adiabatic states

$$
\hat{H}(\mathbf{r} ; \mathbf{R})\left|\phi_{j}\right\rangle=\varepsilon_{j}(\mathbf{R})\left|\phi_{j}\right\rangle
$$

obtained at fixed positions of the classical coordinates $\mathbf{R}$. The wavefunction of the quantum subsystem at any instant of time can be written as a superposition of the adiabatic states (3.5) as

$$
|\Psi(\mathbf{r} ; t)\rangle=\sum_{j} c_{j}(t)\left|\phi_{j}\right\rangle
$$

where the $c_{j}(t)$ are the time-dependent expansion coefficients, with $\sum_{j}\left|c_{j}(t)\right|^{2}=1$. 
The difference between MFA and SH is the way of propagating the classical trajectories, or how the feedback from the quantum to the classical subsystem is taken into account.

\subsubsection{Mean Field (Ehrenfest) Approximation (MFA)}

The MFA method can be derived as a classical limit of the time-dependent Hartree or timedependent self-consistent field method (TDSCF). Here the total wave function is separated into a part belonging to the fast (quantum) particles and a slow (classical) particle wave function. The motion of the particles will interact by their average fields, so the fast particles will move in the mean field of the slow particles and vice versa. The slow particles move via classical mechanics on a potential energy surface given by the expectation value of the fast particle Hamiltonian.

The additional force on the classical particles arises from the weighted average of the quantum states, and the equation of motion for the momenta of the classical particles takes the form

$$
\dot{\mathbf{p}}(t)=-\boldsymbol{\nabla} U+\left(c_{i}^{*} c_{j}+c_{i} c_{j}^{*}\right)\left(\varepsilon_{i}-\varepsilon_{j}\right) \mathbf{d}_{i j}-\left|c_{i}\right|^{2} \nabla \varepsilon_{i}-\left|c_{j}\right|^{2} \nabla \varepsilon_{j}
$$

where the asterisk denotes the complex conjugate, $U(\mathbf{R})$ is the potential energy of the interactions between classical particles, $\mathbf{d}_{i j}(\mathbf{R})$ is the non-adiabatic coupling vector between the states $i$ and $j, \varepsilon_{i, j}(\mathbf{R})$ are the adiabatic energies, see Eq. (3.5), $c_{i, j}(t)$ are the expansion coefficients, see Eq. (3.6), and $\boldsymbol{\nabla}$ is the gradient operator over the classical coordinates $\mathbf{R}$. For the ease of presentation we omit the explicit coordinate and time dependencies on the right hand side of Eq. (3.7).

Thus, as with any mean-field approach, electron correlations are neglected. This deficiency may be particularly severe when one is interested in a low probability channel. In such cases, the MFA path will be quite similar to the major channel trajectory, and will likely be a poor representation of the desired low-probability path, proper description of the correlation between quantum and classical motion requires a distinct classical path for each quantum state. A second 
deficiency of MFA is that it violates microscopic reversibility. This method will be appropriate for systems including only small couplings between the different degrees of freedom, while stronger couplings will lead to decoherence and thus the mean-field is not valid anymore.

\subsubsection{Surface Hopping (SH)}

The SH method invented by Tully in 1971 can be used to implement non-adiabatic transitions in the dynamic simulations of a system. The basic concept can be described as follows: classical trajectories are simulated, usually on an adiabatic state. In regions with two or more potential energy surfaces, the probability of hops from one surface to another is calculated, by this introducing non-adiabatic transitions. After the hopping event, either the trajectory of the new state is traced or a set of new trajectories, following the now occupied different adiabatic surfaces, weighted with a factor complementary to the hopping probability.

In the $\mathrm{SH}$ approach, the forces derived from a single quantum state, are subject to the sudden stochastic hops to the different quantum states. The equation of motion for the classical particles is then

$$
\dot{\mathbf{p}}(t)=-\nabla U-\nabla \varepsilon_{k}
$$

where $k$ labels the current quantum state to which the system is assigned, and the other notations are the same as in Eq. (3.7). Accordingly to the "fewest switches algorithm"43 a state switch from $i$ to $j$ occurs if

$$
P_{i j}(t) \Delta t>\zeta
$$

where $\Delta t$ is the time step of the propagation, $\zeta$ is the uniform random number between 0 and 1 , and

$$
P_{i j}(t)=-\frac{d}{d t} \ln \left|c_{i}\right|^{2}=-\dot{\mathbf{R}} \cdot \mathbf{d}_{j i} \frac{\left(c_{i}^{*} c_{j}+c_{i} c_{j}^{*}\right)}{\left|c_{i}\right|^{2}}
$$

is the probability of hop per unit time. Here $\dot{\mathbf{R}}(t)$ is the classical velocity, $\mathbf{d}_{j i}(t)$ is non-adiabatic coupling vector, and the centered dot denotes the scalar product of vectors. A stochastic surface 
hop from state $i$ to $j$ should be accompanied by the rescaling of the velocity $\dot{\mathbf{R}}$ in the direction of the non-adiabatic coupling vector to conserve the total energy of the system.

The MFA is a self consistent field method, and for the given initial conditions requires propagation of the single classical trajectory, while in the SH approach the feedback of the quantum subsystem on the classical one has an element of uncertainty, see Eq. (3.9), and requires averaging over a swarm of the classical trajectories. However, neither MFA nor SH do accurately describe the non-adiabatic dynamics in a wide range of systems. The former fails in the situation when the energy splitting between the quantum states considerably exceeds the mean heat energy of $k_{B} T$, whereas the $\mathrm{SH}$ has problems with quantum coherence, ${ }^{17}$ and classically forbidden transitions. ${ }^{16}$

\subsection{Reduced Density Matrix Methods}

In general, the state of the open quantum system is described by the Reduced Density Matrix (RDM) rather than by the wave function. There exists two general schemes for the derivation of kinetic equations for the RDM of quantum system. One scheme relies upon partial Wigner transform over classical degrees of freedom and yields the Quantum-Classical Liouville Equation (QCLE), while the other approach, the Redfield theory, starts with an equivalent integro-differential representation of the quantum Liouville-von Neumann equation. The two quantum-classical approaches based on the RDM are discussed below.

\subsubsection{Quantum-Classical Liouville Equation}

The Quantum-Classical Liouville Equation ${ }^{44}$ (QCLE) approach describes the state of the quantum subsystem by the partial density matrix $\sigma(Q ; t)$, parametrically dependent on the phase space coordinates $Q=(\mathbf{p}, \mathbf{r})$ of the classical subsystem, which obeys

$$
\frac{\partial \sigma(Q ; t)}{\partial t}=-\frac{i}{\hbar}[\hat{H}(Q), \sigma(Q ; t)]+\frac{1}{2}\{\hat{H}(Q), \sigma(Q ; t)\}-\frac{1}{2}\{\sigma(Q ; t), \hat{H}(Q)\},
$$


where $\hat{H}(Q)$ is the quantum-classical Hamiltonian, [,] is the commutator and

$$
\{A(Q), B(Q)\}=\nabla_{\mathbf{r}} A(Q) \cdot \nabla_{\mathbf{p}} B(Q)-\nabla_{\mathbf{p}} A(Q) \cdot \nabla_{\mathbf{r}} B(Q)
$$

is the Poisson brackets, with $\boldsymbol{\nabla}_{\mathbf{r}, \mathbf{p}}$ denoting the gradient over the coordinates and momenta, respectively. An appealing feature of the QCLE is its simplicity and the correct limits of both pure quantum and pure classical dynamics. In the former case (absence of the classical degrees of freedom), the QCLE reduces to the quantum Liouville-von Neumann equation, whereas in the latter case it becomes the classical Liouville equation for the phase space distribution function.

To discuss this approach in more details, we consider a simple system of two one-dimensional PESs without interaction between them, with the Hamiltonian

$$
\hat{H}(Q)=\left(\begin{array}{cc}
\frac{p^{2}}{2 m}+U_{1}(x) & 0 \\
0 & \frac{p^{2}}{2 m}+U_{2}(x),
\end{array}\right)
$$

where $m$ is the mass of the particle, and $U_{1,2}(x)$ are the corresponding potential energies of the PESs. Then, we obtain from Eq. (3.11), (3.13) the following set of equations for the density matrix elements

$$
\begin{aligned}
\frac{\partial \sigma_{11}(p, x ; t)}{\partial t}= & -\frac{p}{m} \frac{\partial \sigma_{11}(p, x ; t)}{\partial x}+\frac{\partial \sigma_{11}(p, x ; t)}{\partial p} \frac{\partial U_{1}(x)}{\partial x}, \\
\frac{\partial \sigma_{22}(p, x ; t)}{\partial t}= & -\frac{p}{m} \frac{\partial \sigma_{22}(p, x ; t)}{\partial x}+\frac{\partial \sigma_{22}(p, x ; t)}{\partial p} \frac{\partial U_{2}(x)}{\partial x}, \\
\frac{\partial \sigma_{12}(p, x ; t)}{\partial t}= & -\frac{i}{\hbar}\left(U_{1}(x)-U_{2}(x)\right) \sigma_{12}(p, x ; t) \\
& -\frac{p}{m} \frac{\partial \sigma_{12}(p, x ; t)}{\partial x}+\frac{\partial \sigma_{12}(p, x ; t)}{\partial p} \frac{\partial}{\partial x} \frac{U_{1}(x)+U_{2}(x)}{2} .
\end{aligned}
$$

It is readily seen, that the diagonal elements of the partial density matrix describe evolution of the phase space distribution of the corresponding PES, while the equation (3.16) for the offdiagonal element contains the two contributions, evolution of the quantum phase, see the first term on the right hand side of the Eq. (3.16), and the classical motion in a mean-field potential 
$\left(U_{1}(x)+U_{2}(x)\right) / 2$. This mean-field feature inherent in the QCLE is known to be a severe problem of the approach, and, among other items, may even lead to the positivity violation ${ }^{45}$ of the partial RDM.

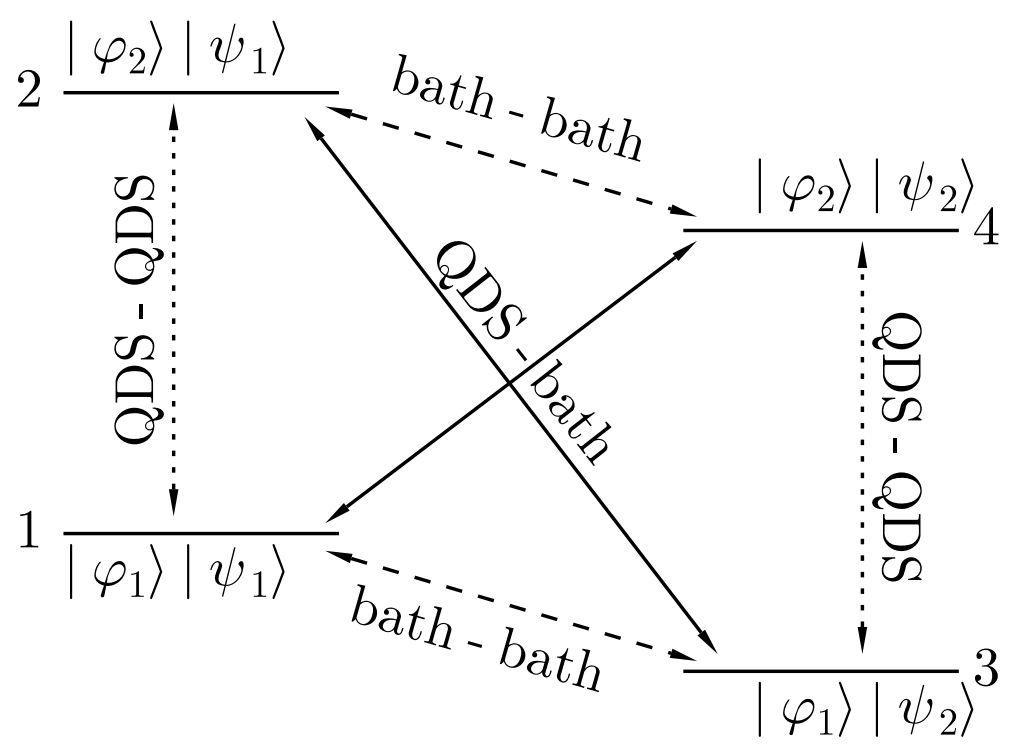

Figure 3.1: Energy levels of a four-level system in the basis of direct product states of QDS $\left(\left|\varphi_{1,2}\right\rangle\right)$ and bath states $\left(\left|\psi_{1,2}\right\rangle\right)$. Dotted arrows show transitions between states of the QDS, dashed arrows - between bath states, while solid arrows show cross transitions between QDS and bath states. This figure was taken from J. Chem. Phys. 119, 2502 (2003).

The origin of the mean-field feature and the associated problems of the QCLE can be understood using a vivid example from Ref., ${ }^{20}$ when both the quantum-dynamical subsystem (QDS) and the classical one (bath) consist of just two states. Fig. 3.1 shows schematically energy levels of the resulting four-level system in the basis of the direct product of the states. Different types of transitions are shown by the different lines, see figure caption. The transitions between the quantum subsystem and the bath (QDS-bath) are very different from the direct transitions inside the quantum subsystem, for example the energy gaps for the transitions $1 \leftrightarrow 4$ and $2 \leftrightarrow 3$ may be not the same.

The creation of the corresponding off-diagonal element is prerequisite for the quantum tran- 
sition, and they are shown below in the total density matrix $\rho$ using the same line types as in Fig. 3.1

$$
\rho=\left(\begin{array}{cccc}
\rho_{11} & \rho_{12} & \rho_{\underline{1}} & \rho_{14} \\
\rho_{21} & \rho_{22} & \rho_{23} & \rho_{24} \\
\rho_{31} 1_{\lrcorner} & \rho_{32} & \rho_{33} & \rho_{34} \\
\rho_{41} & \rho_{42} & \rho_{43} & \rho_{44}
\end{array}\right) .
$$

On the other hand, the reduced density matrix of the quantum subsystem, obtained by taking partial trace over bath states is of the form

$$
\sigma=\left(\begin{array}{cc}
\rho_{11}+\rho_{33} & \rho_{12}+\rho_{34} \\
\rho_{21}+\rho_{43} & \rho_{22}+\rho_{44}
\end{array}\right) .
$$

This means, that off-diagonal elements involving transitions due to QDS-bath and bath-bath interactions do not appear in the reduced density matrix.

Introducing a partial RDM $\sigma(Q ; t)$ in QCLE, we immediately replace the true crosscoherence by the direct coherence inside the quantum subsystem, since in the QCLE there are no other off-diagonal elements. This unphysical replacement of the coherence is a source of the mean-potential feature of the QCLE, and the associated problems.

\subsubsection{Redfield Theory}

Redfield theory ${ }^{46,47}$ is a common name for the group of approaches, where the RDM of the quantum subsystem obeys a kinetic equation of the form

$$
\frac{d \sigma_{i k}(t)}{d t}=-i \omega_{i k} \sigma_{i k}(t)+\sum_{l, m} R_{i k ; l m} \sigma_{l m}(t),
$$

where $\omega_{i k}=\left(E_{i}-E_{k}\right) / \hbar$ is the energy difference between the corresponding states of the quantum subsystem in frequency units, whereas $\hat{\hat{\boldsymbol{R}}}$ is the relaxation tensor describing the effects of the interaction with a heat bath. For the separable system-bath coupling of the form

$$
\hat{W}=\sum_{a} \hat{G}_{a} \hat{F}_{a}
$$


with the operators $\hat{G}_{a}$ and $\hat{F}_{a}$ acting only on the quantum subsystem and the bath, respectively, the relaxation tensor may be represented as ${ }^{46}$

$$
R_{i k ; l m}=\Gamma_{m k ; i l}^{+}+\Gamma_{m k ; i l}^{-}-\delta_{m, k} \sum_{n} \Gamma_{i n ; n l}^{+}-\delta_{i, k} \sum_{n} \Gamma_{m n ; n k}^{-}
$$

where

$$
\begin{aligned}
\Gamma_{m k ; i l}^{+} & =\frac{1}{\hbar^{2}} \sum_{a, b}\left(G_{a}\right)_{m k}\left(G_{b}\right)_{i l}\left(\Theta_{a, b}^{+}\right)_{i l} \\
\Gamma_{m k ; i l}^{-} & =\frac{1}{\hbar^{2}} \sum_{a, b}\left(G_{a}\right)_{m k}\left(G_{b}\right)_{i l}\left(\Theta_{a, b}^{-}\right)_{m k}
\end{aligned}
$$

and

$$
\begin{aligned}
\left(\Theta_{a, b}^{+}\right)_{i k} & =\frac{1}{1+e^{\hbar \omega_{i k} /\left(k_{B} T\right)}} \int_{-\infty}^{\infty} e^{-i \omega_{i k} t}\left\langle F_{a}(t) F_{b}(0)\right\rangle_{c l} d t \\
\left(\Theta_{a, b}^{-}\right)_{i k} & =\frac{1}{1+e^{-\hbar \omega_{i k} /\left(k_{B} T\right)}} \int_{-\infty}^{\infty} e^{-i \omega_{i k} t}\left\langle F_{a}(t) F_{b}(0)\right\rangle_{c l} d t .
\end{aligned}
$$

Here $\left\langle F_{a}(t) F_{b}(0)\right\rangle_{c l}$ is the classical correlation function of the bath operators.

The Redfield theory is valid on the time scale exceeding the characteristic correlation time $\tau_{c}$ of the bath, and requires small off-diagonal elements responsible for the quantum transitions. This is the case in the weak coupling limit

$$
\left(W_{i k} /\left(\hbar \omega_{i k}\right)\right)^{2} \ll 1
$$

or if the following condition holds

$$
\left(W_{i k} \tau_{c} / \hbar\right)^{2} \ll 1
$$

In the latter case the splitting between the energy levels can be arbitrary, including a resonant case of $\omega_{i k}=0$.

To illustrate the underlying principles of the Redfield theory, and following Ref., ${ }^{20}$ we consider the dynamics of the population difference $n(t)=\sigma_{11}(t)-\sigma_{22}(t)$ in a two-level system 
with the splitting between energy levels $\Omega$, constant off-diagonal coupling $W$, and in presence of the phase relaxation with a characteristic time $\tau_{c}$. The kinetic equations for such a model system are of the form ${ }^{20}$

$$
\begin{aligned}
\dot{n}(t) & =-2 W \operatorname{Im}\left[\sigma_{12}(t)\right] \\
\dot{\sigma}_{12}(t) & =\left(i \Omega-1 / \tau_{c}\right) \sigma_{12}(t)+i W n(t) / 2,
\end{aligned}
$$

where for the sake of convenience we set $\hbar=1$, thus working in frequency units. The formal solution of Eq. (3.29) takes the form

$$
\sigma_{12}(t)=\frac{i W}{2} \int_{0}^{t} e^{\left(i \Omega-1 / \tau_{c}\right) \tau} n(t-\tau) d \tau
$$

which, upon substitution into eq. (3.28), yields a closed non-Markovian equation for the population difference

$$
\dot{n}(t)=-W^{2} \int_{0}^{t} e^{-\tau / \tau_{c}} \cos (\Omega \tau) n(t-\tau) d \tau
$$

Both conditions (3.26), (3.27) allow for long times $t \gtrsim \tau_{c}$ to reduce Eq. (3.30) into the differential form, which yields

$$
\dot{n}(t)=-K n(t), \quad K=\frac{W^{2} \tau_{c}}{1+\Omega^{2} \tau_{c}^{2}}, \quad K \tau_{c} \ll 1,
$$

where the last condition ensures small contribution of the neglected time scale of $\tau_{c}$ into the kinetics. If the condition (3.26) is fulfilled, then

$$
K \tau_{c} \ll\left\{\begin{array}{ll}
(W / \Omega)^{2} \ll 1 & \text { if } \quad\left(\Omega \tau_{c}\right)^{2} \gg 1 \\
\left(W \tau_{c}\right)^{2} \ll\left(\Omega \tau_{c}\right)^{2} \ll 1 & \text { if } \quad\left(\Omega \tau_{c}\right)^{2} \ll 1
\end{array},\right.
$$

whereas if the condition (3.27) holds, one has $K \tau_{c} \leq\left(W \tau_{c}\right)^{2} \ll 1$ regardless of the value of $\Omega$.

Redfield theory is a consistent approach with well known applicability limits. Unfortunately, the conditions $t \gg \tau_{c}$, and (3.26), (3.27) are rather restrictive. Indeed, usually the correlation time for the rotational motion of molecules and their fragments in solutions lies in the picosecond time range, for the translational motion (cage effect) it may fall into the nanosecond time 
range. On the other hand, the time scale of the non-adiabatic transitions may be considerably shorter, and their dynamics may exhibit memory effects, which are completely ignored by this approach. Besides, the dynamics of the bath in the framework of the Redfield approach does not depend on the state of the quantum subsystem, and the reaction coordinates have to be treated quantum-mechanically, which restrict practical applications of the approach to the systems with a few reaction degrees of freedom.

\subsection{Non-Markovian Quantum-Classical Approximation}

Recently a novel Non-Markovian Quantum-Classical Approximation ${ }^{19-22}$ (NQCA) has been developed by Neufeld. The applicability criterion of the NQCA requires

$$
\left(W \tau_{b} / \hbar\right)^{2} \ll 1, \quad t \gtrsim \tau_{b}, \quad \tau_{b}=\frac{\hbar}{\pi k_{B} T}
$$

where $W$ is the coupling strength between the quantum subsystem and the bath, $\tau_{b}$ is the lifetime of the quantum correlations, $T$ is the temperature of the heat bath, whereas $\hbar$ and $k_{B}$ are the Planck and the Boltzmann constants, correspondingly. At room temperature $\tau_{b}$ is just $8 \mathrm{fs}$, which allows for the system-bath coupling strength up to $W / \hbar=5 \cdot 10^{13} \mathrm{rad} / \mathrm{ps}$ and the time resolution up to tens of femtoseconds. These applicability limits are considerably wider than that of the Redfield theory, which can directly be obtained from the NQCA in the limit of short correlation time. ${ }^{19}$

The kinetic equations of the NQCA are rather cumbersome, and can be found in Refs. ${ }^{19,20}$ for the case when the bath dynamics does not depend on the state of the quantum subsystem, whereas in Ref. ${ }^{21,22}$ the NQCA was generalized to incorporate the state-specific bath dynamics. The latter has allowed to introduce the classical mechanical treatment of the multidimensional reaction coordinates, ${ }^{22}$ which is of primary importance for realistic systems with many degrees of freedom. The approach can easily be combined with ab initio molecular dynamics and quantum-chemical methods to describe the dynamics of the classical degrees of freedom and 
the topology of the electronic states, respectively. It treats the quantum transitions dynamically with a full account of the detailed balance, quantum coherence and memory effects, which makes the NQCA to be well suited for the ab initio modeling of the non-adiabatic transitions in polyatomic systems.

In the next chapters the approach and its practical applications are described in more details. 


\section{4}

\section{STATISTICAL APPROACH TO}

\section{NON-ADIABATIC TRANSITIONS}

Basically, the applicability limits of NQCA are given by Eq. (3.34). However, in the following we confine ourselves to the case of fast equilibration in the phase space of electron PESs of polyatomic molecules compared to the time scale of the non-adiabatic reaction. The applicability range is similar to the case of non-adiabatic extension ${ }^{34,35}$ to Transition State Theory. Note, however, that we do not assume a one-dimensional reaction coordinate, and the transition probability at surface crossing events as an external parameter.

Under the assumptions made above, the electron PESs can be considered as belonging to the canonical bath, and the state of the system is then described by the set of probabilities $\sigma_{n}(t)$ to be on the given PES, indicated by the subscript. In the following, in order to present equations in a more compact form, we set $\hbar=k_{B}=1$, and, therefore, measure the energies, the couplings and the temperatures in frequency units, where $1 \mathrm{eV}$ and $1 \mathrm{~K}$ correspond to $1.52 \cdot 10^{3} \mathrm{rad} / \mathrm{ps}$ and $0.131 \mathrm{rad} / \mathrm{ps}$, respectively.

Using the MD approach to describe the dynamics of the classical degrees of freedom (phase 
space of the PESs), the time evolution of the probabilities $\sigma_{n}(t)$ obey $^{22}$

$$
\frac{d \sigma_{n}(t)}{d t}=-\sum_{\substack{k=1 \\ k \neq n}}^{N}\left(\left\langle\operatorname{Im}\left[\chi_{n k}^{(n)}(q, t) W_{k n}(q)\right]\right\rangle_{M D}+\left\langle\operatorname{Im}\left[\chi_{n k}^{(k)}(q, t) W_{k n}(q)\right]\right\rangle_{M D}\right)
$$

where the subscripts indicate the PESs, between which the non-adiabatic transitions take place, $W_{k n}$ is the coupling between them, and $\langle\ldots . .\rangle_{M D}$ denotes the average over an ensemble of the MD trajectories of the form

$$
\left\langle\operatorname{Im}\left[\chi_{n k}^{(\alpha)}(q, t) W_{k n}(q)\right]\right\rangle_{M D}=\frac{1}{L} \sum_{l=1}^{L} \operatorname{Im}\left[\chi_{n k}^{(\alpha)}\left(q_{l}^{(\alpha)}(t)\right) W_{k n}\left(q_{l}^{(\alpha)}(t)\right)\right], \quad \alpha=(n, k) .
$$

Here $q_{l}^{(\alpha)}(t)$ represents a set of the phase space coordinates along $l$-th trajectory, evolving either on $n$-th or $k$-th PESs, indicated by the superscript and sampled from the corresponding canonical distribution, $\mathrm{L}$ is in practice a large number of the MD trajectories sufficient to explore the phase space, and $\chi_{n k}^{(\alpha)}\left(q_{l}^{(\alpha)}(t)\right)$ is the set of auxiliary functions (one per trajectory) containing the complete information about quantum coherence and memory effects. In turn, the time evolution of the auxiliary functions is described by the following equation

$$
\begin{aligned}
\frac{d \chi_{n k}^{(\alpha)}\left(q_{l}^{(\alpha)}(t), t\right)}{d t}= & -i \Omega_{n k}\left(q_{l}^{(\alpha)}(t)\right) \chi_{n k}^{(\alpha)}\left(q_{l}^{(\alpha)}(t), t\right)+i W_{n k}\left(q_{l}^{(\alpha)}(t)\right)\left(\sigma_{n}(t)-\sigma_{k}(t)\right) \\
& +i W_{n k}\left(q_{l}^{(\alpha)}(t)\right)\left[\tanh \left(\frac{\varepsilon_{n k}}{2 T}\right)-\frac{i}{2 T} \frac{1}{\cosh ^{2}\left(\frac{\varepsilon_{n k}}{2 T}\right)} \frac{d}{d t}\right]\left(\sigma_{n}(t)+\sigma_{k}(t)(y)\right.
\end{aligned}
$$

with

$$
\Omega_{n k}\left(q_{l}^{(\alpha)}(t)\right)=H_{n}\left(q_{l}^{(\alpha)}(t)\right)-H_{k}\left(q_{l}^{(\alpha)}(t)\right)
$$

to be the difference of the PESs Hamiltonians (vertical energy splitting), $\varepsilon_{n k}$ is the change of the free energy due to transitions from the $n$-th to the $k$-th state expressed in terms of the partition functions of the PES $Z_{n, k}$ as

$$
\varepsilon_{n k}=-T \ln \left(Z_{n} / Z_{k}\right)
$$

and initial conditions of the form

$$
\chi_{n k}^{(\alpha)}\left(q_{l}^{(\alpha)}(0), 0\right)=\frac{1}{2 T} \frac{W_{n k}\left(q_{l}^{(\alpha)}(0)\right)}{\cosh ^{2}\left(\frac{\varepsilon_{n k}}{2 T}\right)}
$$


The auxiliary functions Eq.(4.3) have the property

$$
\chi_{k n}^{(\alpha)}\left(q_{l}^{(\alpha)}(t), t\right)=\left[\chi_{n k}^{(\alpha)}\left(q_{l}^{(\alpha)}(t), t\right)\right]^{*}
$$

where the asterisk denotes the complex conjugate, which allows to avoid unnecessary calculations.

Equations (4.1)-(4.7) constitute the framework of NQCA for systems where the time scale of equilibration in the phase spaces of the PESs is short compared to the time scale of non-adiabatic transitions between them, called the Statistical Approach to the Non-adiabatic Transitions ${ }^{22}$ (SANT). These equations treat the transition dynamically and fully account for detailed balance. Indeed, the stationary solution to Eqs.(4.1)-(4.3) requires that

$$
\frac{d \sigma_{n}(t)}{d t}=0 \quad \Rightarrow \quad \chi_{n k}^{(\alpha)}\left(q_{l}^{(\alpha)}(t), t\right)=0, \quad \frac{d \chi_{n k}^{(\alpha)}\left(q_{l}^{(\alpha)}(t), t\right)}{d t}=0
$$

which is true if

$$
\sigma_{n}=\sigma_{k} e^{-\varepsilon_{n k} / T}
$$

Thus, the canonical equilibrium is indeed the stationary solution to Eqs. (4.1)-(4.7).

To perform the simulation, for any pair of PESs in equation Eq.(4.1) we need the two averages over an ensemble of the MD trajectories, evolving either on one or on another PES. These PESs are physical, unlike the MFA or the QCLE, there are no trajectory propagation along state averaged PESs. To propagate the auxiliary functions (4.3) along the given trajectory, one requires the vertical energy splittings and the PESs couplings. The first term on the right hand side of Eq.(4.3) describes oscillations of the quantum phase of the transitions, whereas the other terms give rise to the quantum phase if the distribution of the $\sigma_{n}$ is a non-equilibrium one. Note, that the term containing the time derivative on the right hand side of Eq.(4.3), together with the initial conditions, Eq.(4.6), preserves positivity of the populations $\sigma_{n}(t)$ at any instant of time, as was demonstrated in Ref. ${ }^{22}$

Thus, the difference between the NQCA and the SH method is that instead of a single hopping trajectory, we need a set of MD trajectories along the PESs involved, without any hops 
between them. The problem cannot be reduced to the evolution of the system along single trajectories, instead the phase spaces (the nuclei coordinates of the corresponding PES) are always represented by a large set of equilibrium trajectories. As compared to the non-adiabatic extension of TST, NQCA does not have the restriction of one-dimensional reaction coordinates, incorporates $a b$ initio treatment of the quantum transitions, and is valid beyond the rate regime, ${ }^{22}$ when quantum coherence effects directly manifest themselves in the reaction dynamics.

In the next chapters the NQCA method will be applied to the simulation of ultraviolet/visible absorption spectra of polyatomic molecules and also to the simulation of photoisomerization processes of polyatomic molecules. 


\section{QUANTUM-CLASSICAL MODELING OF HOT ABSORPTION SPECTRA OF POLYATOMIC MOLECULES}

\subsection{Introduction}

Ultraviolet and visible (UV-vis) absorption spectra provide direct information about the energetics of the electron transitions, which is of primary importance for the modeling and understanding of the reaction dynamics. Usually the experiments on absorption/fluorescence emission are performed at low temperatures (crystals, supersonic jet expansion, for instance) to resolve the vibronic band progressions, thus obtaining extensive information about internal structure of the molecules. The calculation of the electronic spectra for this case may be achieved by the Fermi golden rule approach, where the intensity of the transition is given by the Franck-Condon (FC) overlaps of the initial nuclear wavefunctions with the corresponding vibrational wavefunction of the final electronic state, which requires the evolution of multidimensional integrals. Different methods have been proposed for the evaluation of the Franck-Condon integrals, ${ }^{48,49}$ based 
on the expansion of the final-state coordinate in terms of the initial-state coordinates (or vice versa) and subsequent evaluation of the resulting one dimensional integrals.

However, the approximation of the Franck-Condon integrals as a product of one-dimensional ones requires that the normal modes of the initial and the final PESs are nearly parallel, which is not always the case. In general they are related by the Duschinsky transformation of the form

$$
\zeta^{(1)}=\hat{D} \zeta^{(2)}+\boldsymbol{\gamma}
$$

where $\zeta^{(1)}$ and $\zeta^{(2)}$ are the vector of the normal modes of the corresponding PESs, $\hat{D}$ is the rotational Duschinsky matrix and $\boldsymbol{\gamma}$ is the displacement vector. A large amount of Duschinsky rotation is typical for normal modes belonging to distinct electron states of polyatomic molecules, which does not allow for the efficient calculation of the Franck-Condon integrals.

Moreover, the vast majority of chemical and biological reactions take place at moderate to high temperatures $T \gtrsim 300 \mathrm{~K}$. Hot UV-vis absorption spectra are not only temperature broadened compared to the corresponding low-temperature ones, but may also contain hot bands originating from the FC forbidden transitions. ${ }^{50}$ It has been shown previously ${ }^{50}$ that even for the forbidden transitions - because the molecule does not remain strictly in its equilibrium geometry - there are certain vibrational modes which can introduce intensity to the spectrum. From the molecular geometries and vibrational modes, Franck-Condon factors can be obtained.

The number of Franck-Condon integrals, necessary to describe hot absorption spectra, sharply increases with increasing temperature, due to excitation of low-frequency modes. Therefore, due to the large numbers of Franck-Condon integrals which would have to be calculated, the quantum Fermi's Golden Rule approach becomes hardly applicable.

In this chapter a new method for the ab initio simulation of electronic absorption spectra of polyatomic molecules in the gas phase and in solutions is suggested. The approach utilizes the classical mechanics description and normal modes approximation for the nuclear degree of freedom for each electronic PES, and allows arbitrary Duschinsky rotations. 


\subsection{NQCA and the lineshape of the absorption spectrum}

In the case of two PESs the Hamiltonian of the system interacting with the laser field can be written as

$$
\hat{H}=\hat{H}_{0}+\hat{V}(t)
$$

with

$$
\hat{H}_{0}=\hat{H}_{N}^{(1)}|1\rangle\left\langle 1\left|+\hat{H}_{N}^{(2)}\right| 2\right\rangle\langle 2|,
$$

where $\hat{H}_{N}^{(1,2)}$ are the PESs Hamiltonians depending on the coordinates of the nuclei, $|n\rangle\langle n|$ are the projectors on the $\mathrm{n}$-th electronic state, and

$$
\hat{V}(t)=2 \hat{\boldsymbol{\mu}} \cdot \mathbf{E}_{\mathbf{f}}(t)
$$

is the Hamiltonian of the light-induced coupling between them. Here $\hat{\mu}$ is the transition dipole moment operator and $\mathbf{E}_{\mathbf{f}}(t)$ is the electric field vector

$$
\mathbf{E}_{\mathbf{f}}(t)=\mathbf{E}_{\mathbf{0}} \cos (\omega t)
$$

with $\mathbf{E}_{\mathbf{0}}$ and $\omega$ to be its amplitude and frequency, respectively.

The time evolution of the density matrix of the system may, in general, be described by the following kinetic equation

$$
\frac{d \sigma(t)}{d t}=i \hat{\hat{L}} \sigma(t)-i[\hat{V}(t), \sigma(t)]
$$

where the first term on the right hand side accounts for the time evolution of the system in the absence of laser field, including dissipative processes, with $\hat{\hat{L}}$ being the corresponding Liouville operator. Assuming a vanishing coefficient of the laser field $\left(\mathbf{E}_{\mathbf{0}} \rightarrow 0\right)$, the energy of the system is

$$
E(t)=\operatorname{Tr}\left(\hat{H}_{0} \sigma(t)\right)
$$

with $\operatorname{Tr}$ denoting the trace operation, and, therefore, its time derivative

$$
\frac{d E(t)}{d t}=\operatorname{Tr}\left(\hat{H}_{0} \frac{d \sigma(t)}{d t}\right)
$$


consists of the two contributions, in accordance to Eq.(5.6). The first term on the right hand side of Eq.(5.6) gives rise to the energy flow at the expense of various processes, independent on the light absorption (relaxation, fluorescence emission, etc.), whereas the second term yields the absorption coefficient of the applied laser field

$$
P_{a b s}(t)=\operatorname{Tr}(\hat{P}(t) \sigma(t))
$$

where

$$
\hat{P}(t)=i\left[\hat{V}(t), \hat{H}_{0}\right]
$$

The transition dipole moment operator in Eq.(5.4) couples the distinct electron PESs and can be written as

$$
\hat{\boldsymbol{\mu}}=\hat{\boldsymbol{\mu}}_{\boldsymbol{N}}|1\rangle\left\langle 2\left|+\hat{\boldsymbol{\mu}}_{N}^{\dagger}\right| 2\right\rangle\langle 1|
$$

where the dagger superscript denotes the Hermitian conjugate, $\hat{\boldsymbol{\mu}}$ does not depend on the electron coordinates, but may depend on the coordinates of the nuclei. Then, substituting Eq.(5.5) and Eq.(5.11) in Eq.(5.4), and evaluating the commutator in the Eq.(5.10) we obtain

$$
\hat{P}(t)=-2 i\left(\left(\hat{H}_{N}^{(1)} \hat{\boldsymbol{\mu}}_{\boldsymbol{N}}-\hat{\boldsymbol{\mu}}_{\boldsymbol{N}} \hat{H}_{N}^{(2)}\right)|1\rangle\langle 2|-\text { h.c. }\right) \cdot \mathbf{E}_{\mathbf{0}} \cos (\omega t)
$$

with h.c. denoting the hermitian conjugate. The explicit dependence of the light-induced coupling, see Eq.(5.4), can be eliminated when passing into the rotating coordinate frame by the following unitary transformation

$$
\tilde{\sigma}(t)=\hat{U}(t) \sigma(t) \hat{U}^{\dagger}(t)
$$

with

$$
\hat{U}(t)=e^{i \omega t / 2}|1\rangle\left\langle 1\left|+e^{-i \omega t / 2}\right| 2\right\rangle\langle 2|
$$

where $\omega$ is the frequency of the laser field, see Eq.(5.5). In the rotating frame the Hamiltonian takes the form

$$
\hat{H}_{r}=\left(\hat{H}_{N}^{(1)}+\frac{\omega}{2}\right)|1\rangle\left\langle 1\left|+\left(\hat{H}_{N}^{(2)}-\frac{\omega}{2}\right)\right| 2\right\rangle\langle 2|,
$$


and, neglecting the counter-rotating component of the linearly polarized laser field, Eq.(5.5), the Hamiltonian of the coupling, Eq.(5.4), is transformed to

$$
\hat{V}_{r}=\hat{\boldsymbol{\mu}} \cdot \mathbf{E}_{\mathbf{0}}=\left(\hat{\boldsymbol{\mu}}_{\boldsymbol{N}}|1\rangle\left\langle 2\left|+\hat{\boldsymbol{\mu}}_{\boldsymbol{N}}^{\dagger}\right| 2\right\rangle\langle 1|\right) \cdot \mathbf{E}_{\mathbf{0}} .
$$

Thus, the calculation of the absorption spectra reduces in the rotating frame to the standard problem of the non-adiabatic transitions. The coupling operator, Eq.(5.16), now does not depend on time explicitly, but the splitting between the PESs in the rotating frame varies with the frequency of laser field in accordance with Eq.(5.15).

To express the absorption coefficient, Eq.(5.9), through the density matrix in the rotating frame we substitute the inverse relation

$$
\sigma(t)=\hat{U}^{\dagger}(t) \tilde{\sigma}(t) \hat{U}(t)
$$

into the right hand side of Eq.(5.9), use the cyclic permutation under the trace, and, neglecting the fast oscillation component of the absorption coefficient we obtain

$$
P_{a b s}(t)=\operatorname{Tr}\left(\hat{P}_{r} \tilde{\sigma}(t)\right)
$$

where

$$
\hat{P}_{r}=-i\left(\left(\hat{H}_{N}^{(1)} \hat{\boldsymbol{\mu}}_{N}-\hat{\boldsymbol{\mu}}_{N} \hat{H}_{N}^{(2)}\right)|1\rangle\langle 2|-\text { h.c. }\right) \cdot \mathbf{E}_{\mathbf{0}}
$$

Assuming that the absorption is counterbalanced by spontaneous emission or radiationless relaxation into the ground state, the stationary absorption

$$
P_{\infty}(\omega)=\lim _{\delta \rightarrow 0} \int_{0}^{\infty} P_{a b s}(t) e^{-\delta t} d t
$$

In the case of a vanishing power of the absorbed light (linear response regime), the stationary absorption coefficient can be written as

$$
P_{a b s}(t)=\left(\left\langle\operatorname{Im}\left[\Omega(q) W(q) \chi^{(1)}(q, t)\right]\right\rangle_{M D}+\left\langle\operatorname{Im}\left[\Omega(q) W(q) \chi^{(2)}(q, t)\right]\right\rangle_{M D}\right) \gamma(\omega)
$$


where

$$
\gamma(\omega)=1+\tanh \left(\frac{\omega+\varepsilon}{2 T}\right)
$$

is the frequency dependent factor with

$$
\varepsilon=-T \ln \left(Z_{1} / Z_{2}\right)
$$

being the change of free energy due to the transition between $S_{0}$ and $S_{1}$, expressed via the statistical sums of the PESs indicated by the subscript. Similar to Eq.(4.1) and Eq. (4.2) the MD averages over the ensembles of the equilibrium trajectories are

$\left\langle\operatorname{Im}\left[\Omega(q) W(q) \chi^{(\alpha)}(q, t)\right]\right\rangle_{M D}=\frac{1}{L} \sum_{l=1}^{L} \operatorname{Im}\left[\Omega\left(q_{l}^{(\alpha)}(t)\right) W\left(q_{l}^{(\alpha)}(t)\right) \chi^{(\alpha)}\left(q_{l}^{(\alpha)}(t)\right)\right], \quad \alpha=(1,2)$

where $L$ is a large number of MD trajectories,

$$
W\left(q_{l}^{(\alpha)}(t)\right)=\boldsymbol{\mu}_{\boldsymbol{N}}\left(q_{l}^{(\alpha)}(t)\right) \cdot \mathbf{E}_{\mathbf{0}}
$$

is the light-induced coupling in the rotating coordinate frame, whereas

$$
\Omega\left(q_{l}^{(\alpha)}(t)\right)=H_{N}^{(2)}\left(q_{l}^{(\alpha)}(t)\right)-H_{N}^{(1)}\left(q_{l}^{(\alpha)}(t)\right)
$$

is the vertical energy splitting between the PESs along the given trajectory, respectively.

The structure of Eq. (5.21) needs additional discussion. Two terms in brackets on the right hand side are closely related to the evolution of the quantum phase of the transition, $W(q)$ is the coupling term and represents itself scalar product between the vectors of the electric field amplitude, $\mathbf{E}_{\mathbf{0}}$, and of the transition dipole moment of the molecule, $\boldsymbol{\mu}_{N}\left(q_{l}^{(\alpha)}(t)\right)$, whereas the auxiliary functions $\chi^{(1,2)}(q, t)$ contain complete information about quantum coherence and memory effects, and obey

$$
\frac{d \chi^{(\alpha)}\left(q_{l}^{(\alpha)}(t), t\right)}{d t}=i\left(\Omega\left(q_{l}^{(\alpha)}(t)\right)-\omega\right) \chi\left(q_{l}^{(\alpha)}(t), t\right)+i W\left(q_{l}^{(\alpha)}(t)\right)
$$

where $\omega$ is the frequency of the absorbed light, see also text after Eq. (4.2) for more details. 
Here also the contributions from the single trajectories may be accumulated one-by-one, which is possible as usual in the linear response regime. Eq. (5.21) has a transparent physical meaning. The contribution to the rate of the non-adiabatic transitions between the PESs at a given point of the phase space is proportional to the imaginary part of the product between the coupling and the auxiliary function. Similarly, the contribution to the rate of the energy of absorption at the same point of the phase space is equal to the rate of the transition multiplied by the vertical energy splitting between the PESs, see Eq. (5.21).

\subsection{Model and simulation procedure}

In the present model the harmonic normal mode approximation for the PESs of both the ground and excited states is employed. Although the anharmonicity and the mode couplings play an important role, they can hardly be obtained from the quantum chemical calculations of polyatomic molecules.

In the harmonic normal mode approximation, the phase space of the PESs can be described in terms of the corresponding equilibrium configuration, the normal modes displacements

$$
\zeta^{(\alpha)}=\left\{\zeta_{1}^{(\alpha)}, \zeta_{2}^{(\alpha)}, \ldots\right\}
$$

where the superscript $\alpha$ labels the PES, and their momenta. The transformation matrix between the normal modes displacements and the mass-weighted Cartesian displacements from the equilibrium positions of atomic centra is directly available from the Hessian calculation by quantum-chemical software packages. Simulation of the multi-dimensional classical trajectories on the PESs and of the vertical energy splitting, necessary for the ab initio modeling of both the UV/vis absorption spectra and the non-adiabatic photoisomerization reaction, is outlined in the next Section 5.4.

The dependence of the transition dipole moment on nuclear coordinates was assumed to be 
linear with respect to the normal mode displacements, i.e.

$$
\boldsymbol{\mu}_{\boldsymbol{N}}(t)=\boldsymbol{\mu}_{\boldsymbol{N}}^{(0)}+\left.\sum_{k} \frac{\partial \boldsymbol{\mu}_{\boldsymbol{N}}(\boldsymbol{\zeta})}{\partial \zeta_{k}}\right|_{\zeta_{k}=0} \zeta_{k}(t)
$$

Here $\boldsymbol{\mu}_{N}{ }^{(0)}$ is the transition dipole moment (TDM) calculated at the optimized PES geometry, while the derivatives with respect to the normal mode coordinates in the second term on the right hand side of Eq. (5.29) can be obtained numerically generating a small displacement along each normal mode followed by the single point TDM calculation. Often the first term on the right hand side plays a dominant role, so that the absorption spectra can be simulated within the Condon approximation, when TDM is assumed to be independent on the nuclear coordinates. Exceptions are highly symmetric molecules, for instance benzene with $\mu_{N}{ }^{(0)}=0$. The lightinduced coupling between the PESs among other factors depends on the relative orientation of the molecule to the direction of the electric field $\mathbf{E}_{\mathbf{0}}$, see Eq. (5.25). This orientation is not fixed, and the average angular velocity can be estimated from the average energy per rotational degree of freedom of $k_{B} T$ and the moments of inertia of the molecule. The larger the molecule, the slower it rotates. In our simulations we neglect the effect of the rotational broadening of the UV-vis absorption spectra, and set up random but fixed orientation of the molecule with respect to the laser field in the beginning of every trajectory run.

\subsection{Calculation of multi-dimensional trajectories and verti- cal energy splittings}

In the harmonic normal mode approximation the multi-dimensional trajectory on the PES may be described in terms of the equilibrium configuration, normal modes displacements and momenta. The former is obtained by the geometry optimization procedure, whereas the frequencies of the normal modes, and the transformation matrix from the mass-weighted cartesian coordinates to the normal modes are directly available from the hessian calculation, both calculations 
were performed using CASSCF method and the GAMESS US software. The initial conditions for the normal modes oscillators were specified as follows. First, for every normal mode we sample its energy from the canonical distribution by setting ${ }^{51}$

$$
E_{k}=-k_{B} T \ln \xi
$$

where $\xi$ is the uniform random variate on $(0,1)$. Then, one has the following equations for the time evolution of the mass-weighted displacements

$$
\zeta_{k}(t)=\frac{\sqrt{2 E_{k}}}{\omega_{k}} \cos \left(\omega_{k} t+\varphi_{k}\right)
$$

and of the corresponding momenta

$$
\dot{\zeta}_{k}=\sqrt{2 E_{k}} \sin \left(\omega_{k} t+\varphi_{k}\right)
$$

where $\omega_{k}$ and $\varphi_{k}$ being the frequency, and the initial phase of the k-th normal mode oscillator, respectively. The initial phase for the oscillators was taken random from the interval $[0,2 \pi)$. Thus at any instant of time we are able to calculate the set of normal modes displacement and momenta for the given trajectory, using Eqs.(5.31-5.32).

Calculation of the vertical energy splitting between the PESs along the given trajectory requires 3 quantities, the potential energy for the first PES, counted from its energy minimum, the same quantity for the second PES, and the vertical energy splitting $\left(\hbar \Omega_{0}\right)$ between the minima of the PESs. The latter can be obtained from the ab initio quantum chemistry calculations and spectroscopic data, see Section 5.5 for more details. The vertical energy splitting (in frequency units) can be written as

$$
\Omega(t)=\frac{1}{2} \sum_{n}\left(\omega_{n}^{(2)} \zeta_{n}^{(2)}\right)^{2}-\frac{1}{2} \sum_{k}\left(\omega_{k}^{(1)} \zeta_{k}^{(1)}\right)^{2}+\Omega_{0}
$$

where $\Omega_{0}$ is counted from the minimum of the second PES, and is positive, if the energy minimum of the second PES lies above the minimum of the first one. 
In the following, for the definiteness, we assume that the trajectories evolve on the first PES. The equilibrium geometry, the set of the normal modes, and their frequencies of the second PES are different from those of the first PES, therefore, we have to find how the current molecular configuration is represented by the set of the normal modes displacements on the second PES. This is done in 3 steps. First, we restore the cartesian coordinates of the molecules from the known set of the normal modes displacements, the transformation matrix between the normal modes and the mass-weighted cartesian displacements, and the equilibrium configuration. Second, we rotate the obtained cartesian configuration to the Eckart frame of the equilibrium configuration of the second PES, since their orientations obtained by the geometry optimization are slightly different. Third, we transform the obtained cartesian coordinates to the normal modes displacements of the second PES, using its equilibrium configuration and the transformation matrix. Then, Eq. (5.33) is used to obtain the required vertical energy splitting. Note, that the above procedure works for arbitrary Dushinsky rotation in the molecule.

\subsection{Estimation of the relative energetics of the PESs for trans- stilbene and p-coumaric acid}

Accurate estimation of the energy splittings between the PESs is of fundamental importance for the modeling and understanding of nonadiabatic reactions. This quantity is also frequently used to examine the quality of quantum chemical methods, whereas an account for PES-dependent electron correlation energy represents considerable difficulties for the perturbation theories employed there. In particular, the vertical excitation energy for trans-stilbene was calculated in Ref. $^{52}$ using CASSCF/CASPT2 approach to be $4.07 \mathrm{eV}$, which is indeed close to the $0-0$ transition energy of $4.00 \mathrm{eV} .{ }^{53}$ On the other hand, the molecule upon photoexcitation undergoes geometric changes which reduces its energy and may lead to considerably lower $0-0$ transition energy than the vertical one. 
To estimate the difference between the $0-0$ and the vertical transition energies for transstilbene calculations done in Ref. ${ }^{52}$ have been repeated and the vertical excitation energy to the single ${ }^{1} B_{u}(H L)$ and double ${ }^{1} A_{g}(Z)$ excited states have been obtained using CASPT2 approach. The latter excited state is recognized to play a fundamental role in the process of photoisomerization of trans-stilbene, as the radiationless transition into the ground state takes place in the twisted conformation characterized as the double excited one. ${ }^{26-29}$ Then the geometries for the excited states of interest have been optimized and the calculations have been repeated. The results are summarized in Table 5.1.

\begin{tabular}{l|c|c|c} 
electron & \multicolumn{3}{|c}{ optimal geometries } \\
state & ${ }^{1} A_{g}(G)$ & ${ }^{1} B_{u}(H L)$ & ${ }^{1} A_{g}(Z)$ \\
\hline${ }^{1} A_{g}(G)$ & 0.00 & 0.12 & 0.72 \\
${ }^{1} B_{u}(H L)$ & 4.05 & 3.71 & 4.39 \\
${ }^{1} A_{g}(Z)$ & 4.78 & 4.18 & 4.06 \\
\hline
\end{tabular}

Table 5.1: Vertical excitation energies, ${ }^{54}$ corrected by LS(0.3 a.u.)-CASPT2. The energies are in electron-volts and counted from the energy of the ground $\left({ }^{1} A_{g}(G)\right)$ state at its optimal geometry.

It is well understood that the vertical excitation energies predicted by quantum chemical calculations are usually not very accurate, but the energy profiles along the given PES are well reproduced. Therefore, we estimate from the data shown in Table 5.1 the difference between the $0-0$ and the vertical excitation energy for ${ }^{1} B_{u}(H L)$ state to be $0.34 \mathrm{eV}$, which means that the vertical excitation energy is about $4.00+0.34=4.34 \mathrm{eV}$, where $4.00 \mathrm{eV}$ is the experimentally measured $0-0$ transition in the molecular beam. ${ }^{53,55,56}$ The above analysis confirms the intrinsic error of CASPT2 of about $0.3 \mathrm{eV}$ in predicting the vertical excitation energies, and the fact that it usually overestimates the corrections to the CASSCF energies. ${ }^{57-59}$

The $1{ }^{1} A_{g} \rightarrow 3{ }^{1} A_{g}$ transition was assumed ${ }^{60}$ to be responsible for the absorption band with a maximum at $234 \mathrm{~nm}$ or $5.3 \mathrm{eV}$ in the two-photon spectrum of trans-stilbene dissolved in hexane, 
which should be close to the vertical excitation energy to ${ }^{1} A_{g}(Z)$ state. On the other hand, Table 5.1 shows that the difference between the $0-0$ and the vertical excitation energies is about $0.72 \mathrm{eV}$, which yields an estimate of $5.30-0.72=4.58 \mathrm{eV}$ for the $0-0$ transition ${ }^{1} A_{g}(G) \rightarrow$ ${ }^{1} A_{g}(Z)$. The results are summarized in the Table 5.2, where the energies are corrected to be in agreement with the spectroscopical data.

\begin{tabular}{l|c|c|c} 
electron & \multicolumn{3}{|c}{ optimal geometries } \\
state & ${ }^{1} A_{g}(G)$ & ${ }^{1} B_{u}(H L)$ & ${ }^{1} A_{g}(Z)$ \\
\hline${ }^{1} A_{g}(G)$ & 0.00 & 0.12 & 0.72 \\
${ }^{1} B_{u}(H L)$ & 4.34 & 4.00 & 4.74 \\
${ }^{1} A_{g}(Z)$ & 5.30 & 4.70 & 4.58 \\
\hline
\end{tabular}

Table 5.2: The corrected energies of the selected electronic states of trans-stilbene. The energy profiles along the given state were taken from the Table 5.1. The relative shifts between the profiles were adjusted to fit the spectroscopical data. For ${ }^{1} B_{u}(H L)$ state we used the known value of $4.00 \mathrm{eV}$ for the $0-0$ transition, whereas for ${ }^{1} A_{g}(Z)$ state we estimated the corresponding vertical excitation energy of $5.30 \mathrm{eV}$. As in the Table 5.1 the energies are counted from the energy of the ground state at its optimal geometry.

Figure 5.1 shows the relative energetics of the PESs of trans-stilbene, where both the vertical excitation energies and the energies of the PESs minima, counted from the minimum of the ground state PES, are shown. The (local) energy minimum of $S_{2}$ PES lies above those of $S_{1}$ state. 


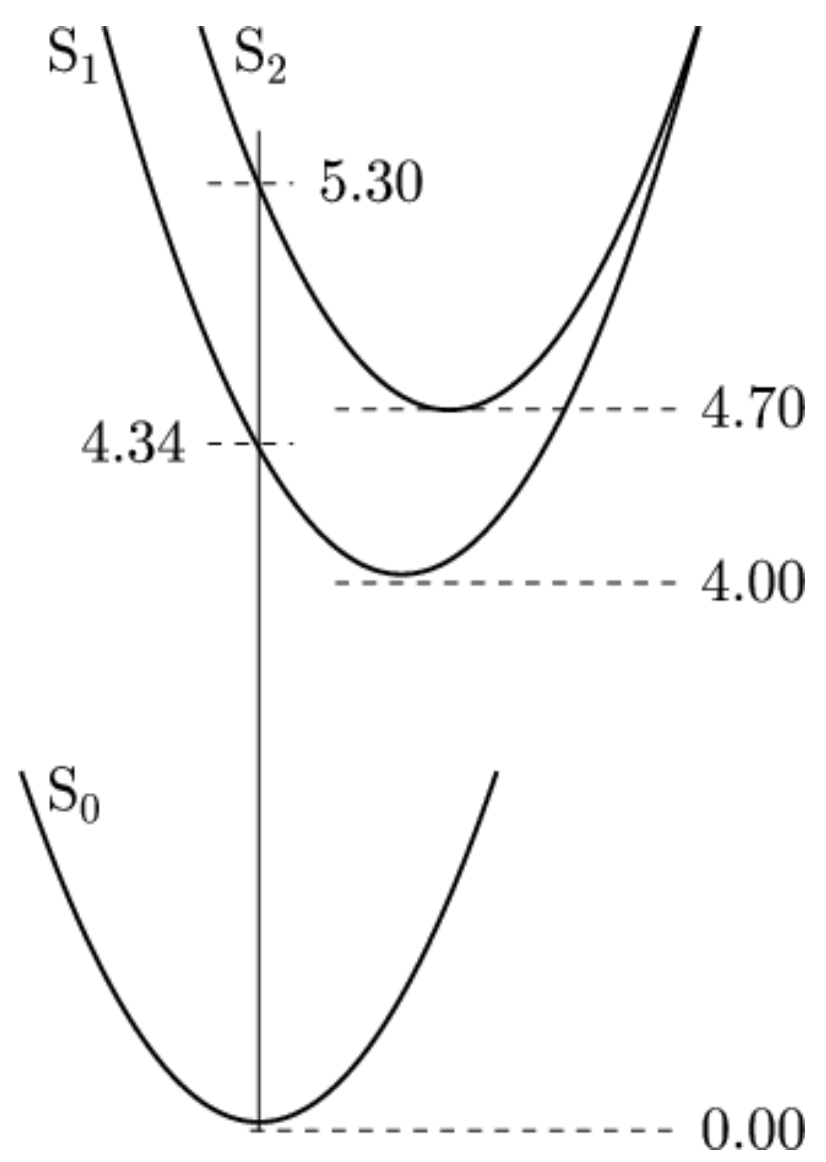

Figure 5.1: The relative energies of the PESs of trans-stilbene, in electron-volts. Both the PESs energy minima and the vertical excitation energies are shown, the data are taken from the Table 5.2.

Similar approach was employed to estimate the relative energetics of the PESs of p-coumaric acid. The energy difference between the minima of the ground and the $S_{1}$ PES is taken to be $33200 \mathrm{~cm}^{-1}$ or $4.11 \mathrm{eV}(0-0$ transition), which is available from the spectroscopical data. ${ }^{61}$ The two-photon absorption spectrum is broad with a maximum located at approximately $38400 \mathrm{~cm}^{-1}(4.76 \mathrm{eV})$, and is assumed to be the vertical energy splitting between the ground and the $S_{2}$ PESs, taken at the optimal geometry of the ground state. Thus, in order to obtain the relative energy difference between the $S_{2}$ and the $S_{1}$ PESs, we have to estimate the energy difference along the $S_{2}$ state at the optimal geometry of the ground state and at its optimal geometry. This quantity was found from our ab initio calculations on CASSCF(10e/8o) level to be $1.34 \mathrm{eV}$, and, therefore, the resulting energy difference between the minima of $S_{2}$ 
and $S_{1}$ states is $-0.69 \mathrm{eV}$, which also means that the minimum of $S_{2}$ state lies below that of $S_{1}$ state, contrary to the findings for trans-stilbene. Note, that we have avoided the direct quantumchemical calculation of the energy difference between the distinct electronic PESs, which is known to be a considerable problem even for the most sophisticated approaches, only the energy difference along the same PES was used. The obtained results are summarized on Fig. 5.2.

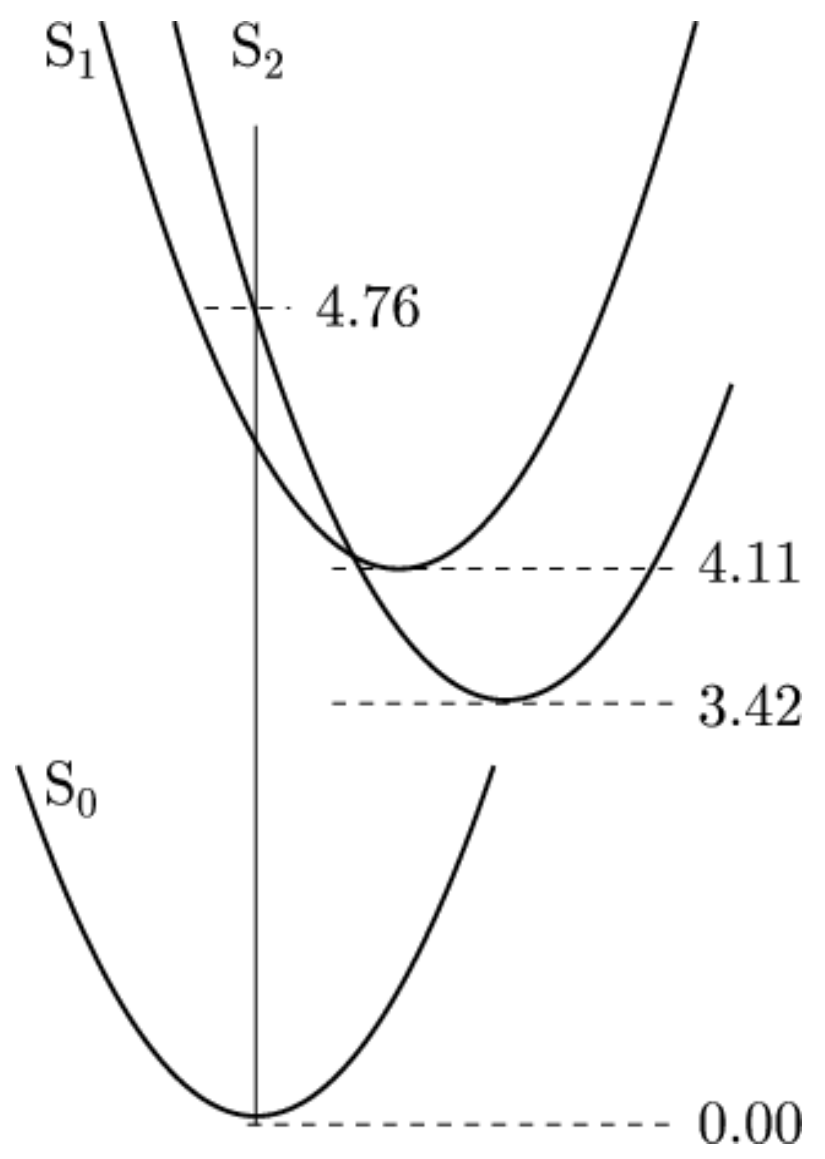

Figure 5.2: The relative energies of the PESs of p-coumaric acid, in electron-volts. Both the PESs energy minima and the vertical excitation energy $S_{0} \rightarrow S_{2}$ are shown. 


\subsection{Results - Solvent influence on the transition energy gap}

The fundamental difference between the NQCA and the other quantum-classical approaches to the non-adiabatic transitions is that the dynamics both along the ground and the excited PESs contribute to the evolution of the quantum phase of the non-adiabatic transitions, and, therefore, to the absorption spectra, see Eq.(5.21). These two components have distinct maxima, corresponding to the absorption and emission ones, whereas a proper absorption spectrum part is separated out by the frequency dependent factor $\gamma(\omega)$, see Eqs. (5.21)-(5.23). At low temperatures these two contributions to the spectral line shape are usually well separated, however, at high temperatures their overlap may become important and visible on the spectra.

A vivid example of this kind is the absorption spectrum of trans-stilbene, dissolved in hexane at room temperature $(T=293 \mathrm{~K})$, taken from the freely available PhotochemCAD database. ${ }^{62}$ For the matter of convenience the fluorescence emission spectrum (excitation wavelength $290 \mathrm{~nm}$ ) is also plotted ibidem. The spectra were scaled to have similar amplitudes, see Fig. 5.3.

The absorption spectrum has a visible shoulder on its left side at the excitation energy of about $3.9 \mathrm{eV}$, a similar one exists on the emission spectrum, see Fig. 5.3. They appear because the two broad overlapping contributions in the Eq. (5.21), discussed above, form a saddle between them. Besides, the width of this saddle at a given temperature proves to be larger than the width of the stepwise-like factor $\gamma(\omega)$, see Eq. (5.22), which leads to the appearance of the well visible shoulders in the spectra shown on Fig. 5.3. But it also means, that the initial rise of the absorption spectrum has to be well described just by the functional dependence of $\gamma(\omega)$, and it is indeed perfectly fitted by Eq.(5.22) with $\varepsilon=-3.81 \mathrm{eV}$ and $T=293 \mathrm{~K}$, see Fig. 5.3. Among other things, it gives an accurate estimate of the free energy change upon photo-excitation including both $0-0$ transition energy gap and the corresponding entropic part.

It is well known that the surrounding media may considerably affect the energy splitting 


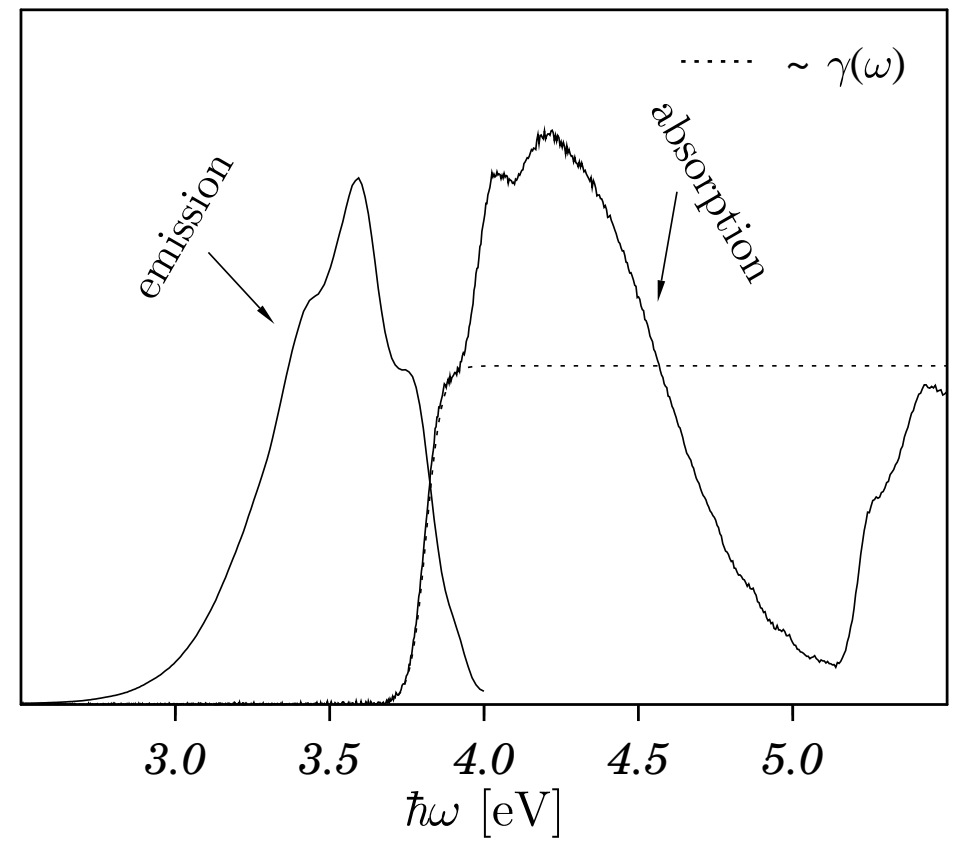

Figure 5.3: Fluorescence emission and absorption spectra of trans-stilbene dissolved in hexane at room temperature. The spectra were scaled to have similar amplitudes. The fit of the initial rise of the absorption spectrum by the stepwise-like function gamma, see Eq.(5.22), with the parameters $\varepsilon=-3.81 \mathrm{eV}$ and $T=293 \mathrm{~K}$ is shown by the dotted line. The experimental absorption and emission spectra of stilbene molecule was taken from the freely available PhotochemCAD database. $^{62}$

between the distinct electronic states, the PES-specific dipole-dipole interaction with solvent molecules is one source of the influence. On the other hand, a direct measurement of the energy gap of the $0-0$ transition requires high resolution experiments usually performed at low temperatures, which can hardly be applied to investigate solvent effects on the energies of the electronic transitions. In the case of trans-stilbene, where we understand the origin of the shoulders, the accurate value of the transition free energy gap can be directly extracted from the hot absorption spectra as well. Indeed, applying the above procedure to the gas phase spectra shown on Fig. 5.4, where we expect no or little influence of the surrounding media, we obtain $\varepsilon=3.99 \mathrm{eV}$, which is in excellent agreement with the experimental value of $4.00 \mathrm{eV}$ $(310.23 \mathrm{~nm})$, obtained for the jet-cooled molecule, ${ }^{53}$ and for non-polar benzene as the solvent, ${ }^{63}$ where it is found to be $3.98 \mathrm{eV}$. Thus, one may conclude that hexane as the solvent reduces the splitting between the ground and the first excited state by $0.18 \mathrm{eV}$ compared to the isolated or 
gas-phase trans-stilbene.

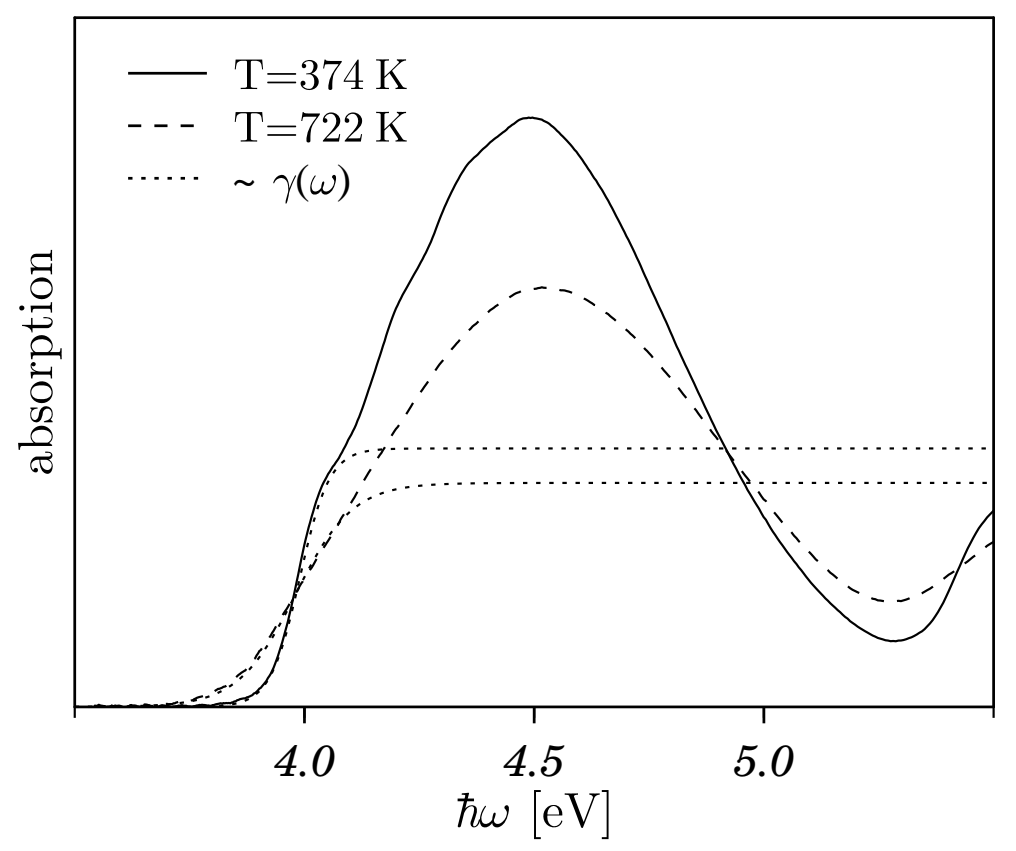

Figure 5.4: Hot absorption spectra of trans-stilbene in gas phase taken at $374 \mathrm{~K}$ (solid line) and $722 \mathrm{~K}$ (dashed line). The corresponding fits of the initial rise by the frequency dependent factor $\gamma(\omega)$ with $\varepsilon=3.99 \mathrm{eV}$ are shown by the dotted lines. The experimental hot absorption spectra of stilbene molecule was taken from the freely available PhotochemCAD database. ${ }^{62}$

Tyrosine, one of the 20 amino acids used by cells in protein synthesis, offers another example. A close look on the absorption spectrum ${ }^{62}$ of tyrosine dissolved in water, shown on Fig. 5.5, reveals a characteristic shoulder, which is supposed to be of the same nature as for the trans-stilbene. Fitting of the initial rise of the spectrum with $\gamma(\omega)$ yields the transition free energy gap of $4.34 \mathrm{eV}$, and it is in a good agreement with the experimental values ${ }^{64,65}$ of $286-288.5 \mathrm{~nm}(4.34-4.30 \mathrm{eV})$ obtained for tyrosine in RNase-S and RNase-A dissolved in a water-glycerol mixture.

The accurate treatment of quantum phase is of fundamental importance for any ab initio quantum-classical approach to non-adiabatic transitions, and the spectral shape is very sensitive to the quantum phase. Note, that neither mean-field nor surface hopping quantum-classical schemes can give satisfactory results when applied to the simulation of the line shape of UVvis absorption spectra. In particular, quantum phase in mean-field approaches evolves on the 


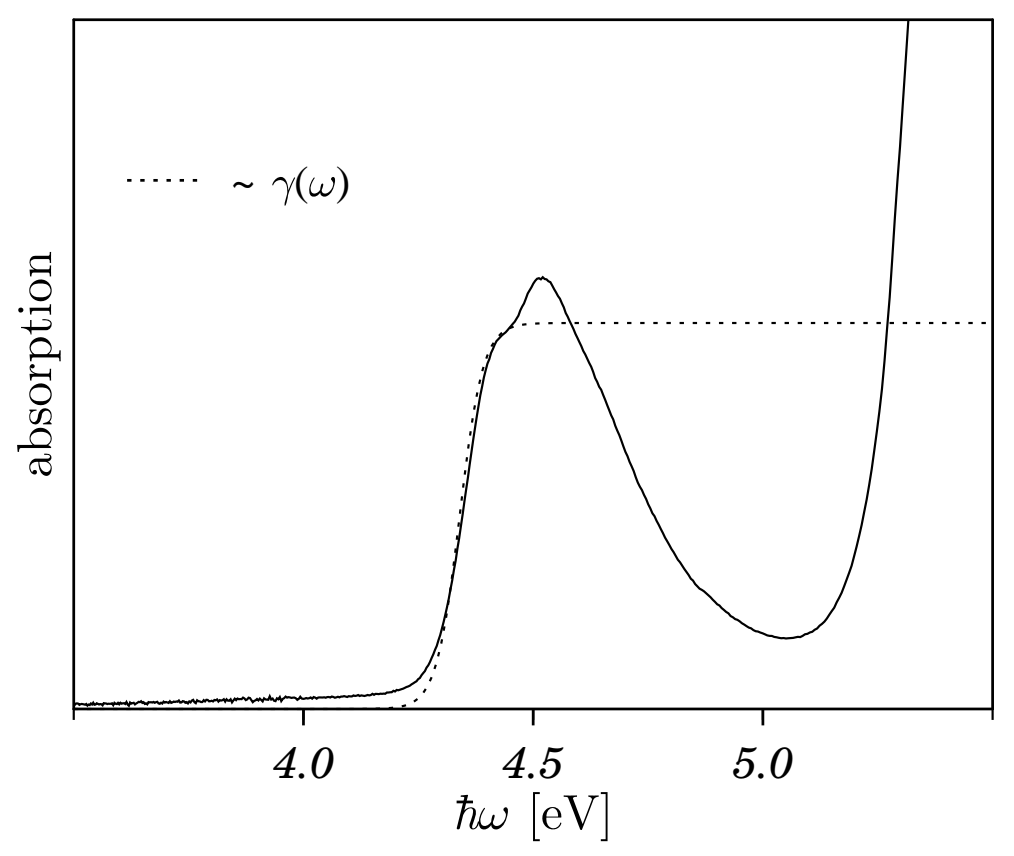

Figure 5.5: The absorption spectrum of tyrosine dissolved in water at room temperature (solid line). The fit of the initial rise by $\gamma(\omega)$ with $\varepsilon=4.34 \mathrm{eV}$ and $T=293 \mathrm{~K}$ is shown by the dotted line. The experimental absorption spectra of tyrosine molecule was taken from the freely available PhotochemCAD database. ${ }^{62}$

averaged PES, which would give instead of spectrally shifted emission and absorption parts just an unphysical line in between. Similarly, surface hopping schemes estimate the dynamics of the populations but not the quantum phase, and, therefore, ab initio simulation of UV-vis absorption spectra can hardly be done. On the other hand, the suggested quantum-classical approach is capable of ab initio modeling of both the population dynamics, and the line shape of the absorption spectra. Its intrinsic feature, that in the quantum-classical approximation the classical dynamics both along the initial and the final PES contribute to the evolution of the quantum phase of transition, seems to be supported by experimental data. The method for direct extraction of the $0-0$ transition energy gap from hot absorption spectra of some polyatomic molecules gives an opportunity to study directly the solvent effects on the energetics of electronic transitions. ${ }^{31}$ 


\subsection{Results - Hot absorption spectra simulations by NQCA}

Ab initio simulation of the absorption spectra was done for trans-stilbene and benzene in the gas phase, employing harmonic normal mode approximation as described in Sec. 5.3. The establishing of the canonical equilibrium in the phase spaces of PESs is controlled by the molecular collisions and by the IVR processes. In view of much larger splitting between the ground and the excited electron states compared to the mean heat energy of $k_{B} T$, initially the molecules are in the ground state, and the corresponding canonical distribution is not affected much by the applied laser field, in the assumed case of its vanishing power (linear response regime). The energy distribution of the excited state, involved in the transition, depends on the transferred energy excess. At small energy excess the transferred from the ground state canonical energy distribution is not affected much, however, at larger energy excess the energy distribution of the excited state PES tends to be the micro-canonical one due to the fast IVR, and the light-induced transitions between the PESs take place simultaneously with the energy excess dissipation to the environment. These effects are not accounted for in the frameworks of the employed statistical approach to the non-adiabatic transitions, which assumes the canonical energy distribution in the phase spaces of both PESs. On the other hand, the above problem mainly concerns the fluorescence emission contribution to the spectral shape, see Eq. (5.21), which only gives some contribution to the initial rise of the absorption spectra (shoulder part), and we believe that the quality of the modeling is still sufficient to describe this part of the spectra.

In the case of trans-stilbene the spectrum was modeled in the Frank-Condon approximation assuming a constant transition dipole moment (TDM). On the contrary, benzene is a highly symmetric molecule and has no TDM in its equilibrium configuration, it may appear only in the configurations distorted from the equilibrium one. The spectra look different, the absorption line of trans-stilbene is broad and nearly structureless, whereas the benzene spectrum exhibits a vibronic structure. 


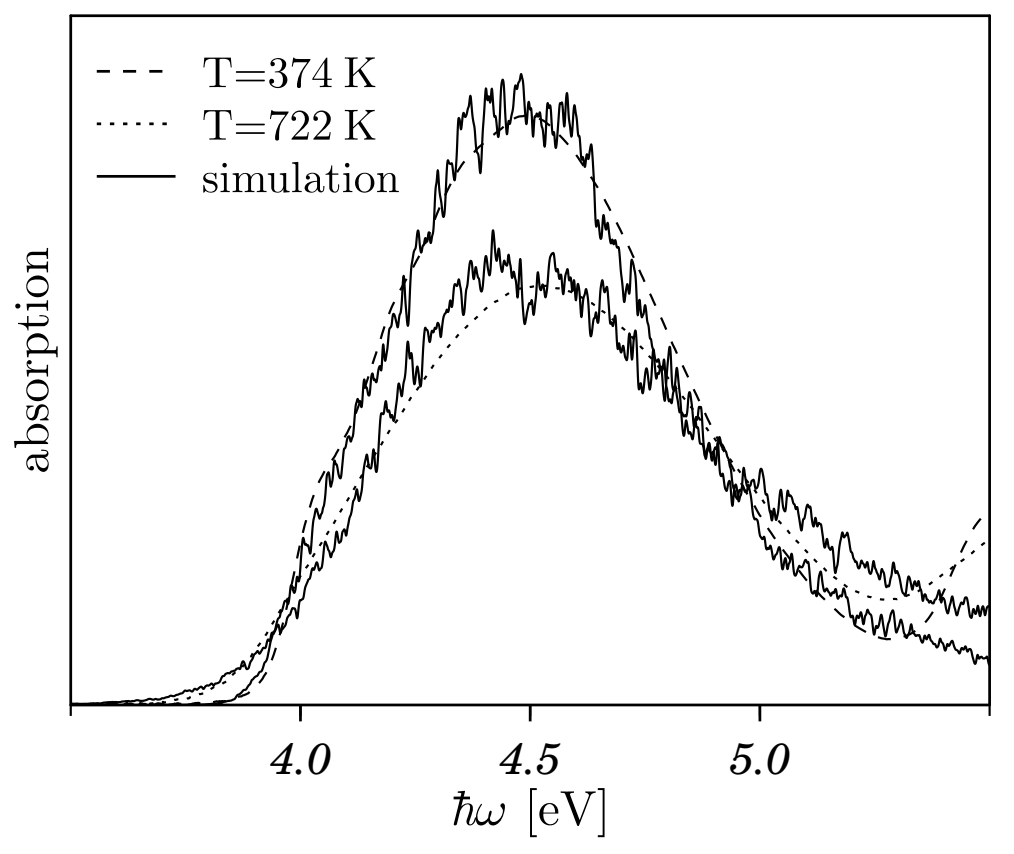

Figure 5.6: The comparison between the experimentally measured hot absorption spectra of trans-stilbene in gas phase at two different temperatures, $T=374 \mathrm{~K}$ (dashed line), and $T=$ $722 \mathrm{~K}$ (dotted line). The corresponding simulated spectra are shown by solid lines. The value of the free energy change upon photoexcitation of $\varepsilon=3.99 \mathrm{eV}$ was used, the simulated spectra were scaled to fit the experiment at $T=374 \mathrm{~K}$. The experimental hot absorption spectra of stilbene molecule was taken from the freely available PhotochemCAD database. ${ }^{62}$

Fig. 5.6 shows a comparison of the simulated and the experimentally measured spectra of trans-stilbene for the two temperatures indicated in the figure. The free energy change $\varepsilon=$ $3.99 \mathrm{eV}$ and the amplitude were taken to fit the experimental spectrum at $T=374 \mathrm{~K}$, the same set of parameters were used to simulate the spectrum at $T=722 \mathrm{~K}$. The configuration space of trans-stilbene consists of 72 degrees of freedom, and the complete sampling of its phase space requires averaging over a large number of trajectories $N$, whereas the remaining fluctuations are suppressed only as $\sim 1 / \sqrt{N}$. In our simulations we used $N=2000$, and $\varepsilon=3.99 \mathrm{eV}$ estimated as discussed above, the simulated spectrum for $T=374 \mathrm{~K}$ was scaled to fit the experimentally observed one, the same set of parameters except the temperature was used for $T=722 \mathrm{~K}$. It can be seen that both the width of the spectra and their relative amplitudes are well reproduced.

Fig. 5.7 shows a comparison between the experimentally measured and the simulated hot spectra of benzene. The vibronic structure of the spectrum at $335 \mathrm{~K}$ is well reproduced, though 


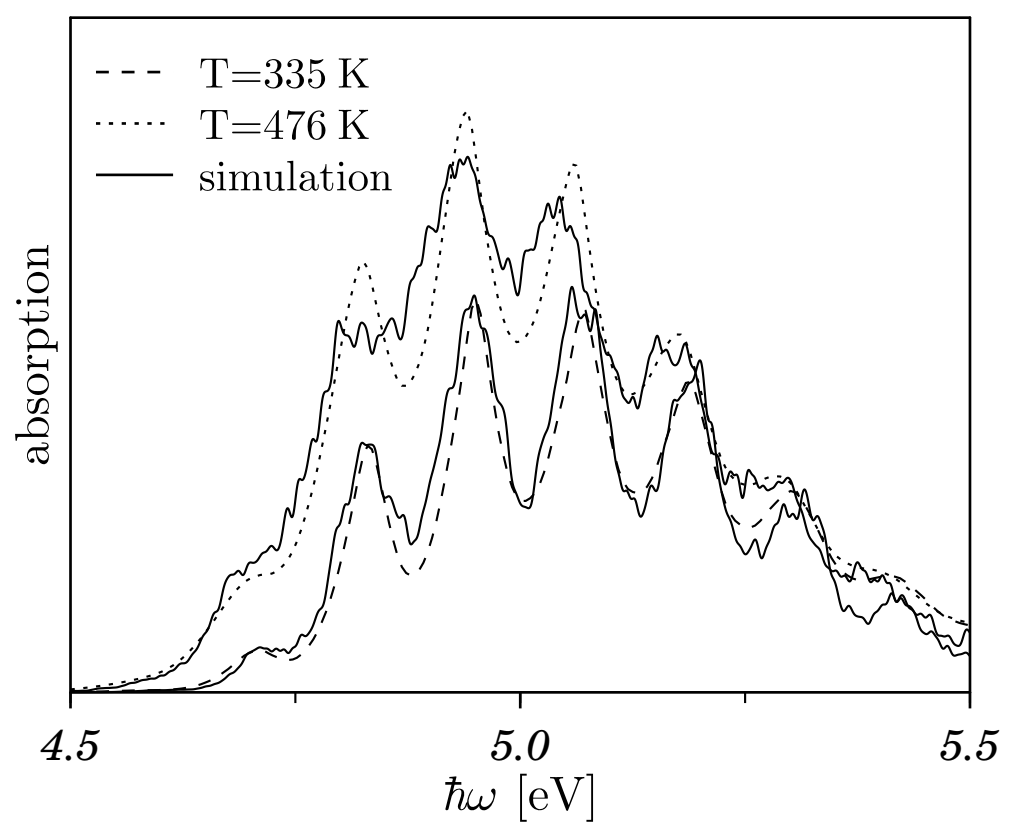

Figure 5.7: The comparison between the experimentally measured hot absorption spectra of benzene, $T=335 \mathrm{~K}$ (dashed line), and $T=476 \mathrm{~K}$ (dotted line). The corresponding simulated spectra are shown by solid lines. The simulated spectra were scaled to fit the experiment at $T=335 \mathrm{~K}$. The experimental hot absorption spectra of benzene molecule was taken from the freely available PhotochemCAD database. ${ }^{62}$

the agreement between the simulation and the experiment at $476 \mathrm{~K}$ degrades, which may be the result of the simplified treatment of multidimensional PESs as the harmonic normal modes approximation. The distinct temperature effect on the intensities of the hot absorption spectra of trans-stilbene and benzene can be explained as follows. Increasing the temperature the amplitude of the distortions from the equilibrium configuration increases, which increases both the induced TDM and the dephasing. In the case of trans-stilbene, the induced TDM is small compared to the equilibrium configuration, whereas the faster dephasing destroys the transition phase, thus broadening the spectrum and reducing its amplitude, see Fig. 5.6. On the other hand, benzene has only an induced TDM and its increase has more effect on the amplitude of the spectrum than the competing more faster dephasing, see Fig. 5.7. 


\subsection{Conclusion}

We proposed a new quantum-classical approach for the ab initio simulation of the hot absorption spectra of polyatomic molecules. It is based on the classical mechanics description of the dynamics along the potential energy surfaces with the quantum mechanical treatment of the transitions between them, and assumes fast equilibration phase space of PESs to the temperature of the surrounding media. The approach differs from the existing ones in the treatment of the quantum transition phase, which is directly related to the shape of the spectra, and of fundamental importance for the modeling of non-adiabatic processes. Unlike the mean-field approximation, where the coherence evolve on a mean-field potential energy surface, the quantum transition phase is given by the sum of the two equally weighted contributions, being propagated along the corresponding PES. Among other things, it may lead to the appearance of the specific shoulders in the absorption spectra, which were indeed found and identified for the case of trans-stilbene and tyrosine. It gives an opportunity for the accurate determination of the $0-0$ transition energy gap for those molecules and for the direct investigation of the solvent influence on the transition free energy, which is of principal importance for the reaction dynamics.

$A b$ initio simulations of the hot absorption spectra in the gas phase were carried out and directly compared to the available experimental data for trans-stilbene and benzene. The harmonic normal modes approximation of both the ground and excited states, obtained by ab initio quantum chemistry methods, was employed to represent multi-dimensional PESs. In the case of trans-stilbene the transition dipole moment was assumed to be constant, whereas in the case of benzene it was calculated by ab initio quantum chemical method generating small displacements along each normal mode. A good agreement between the theoretically modeled and the experimentally measured spectra demonstrates the consistency in the treatment of the transition quantum phase by our quantum-classical approach, and opens broad perspectives to the application of the recently developed NQCA to the $a b$ initio modeling of non-adiabatic reactions in 
complex systems. 
6

\section{QUANTUM-CLASSICAL MODELING}

OF NONADIABATIC

PHOTOISOMERIZATION OF

\section{POLYATOMIC MOLECULES}

\subsection{Introduction}

Two models were suggested to explain the mechanism of photoinduced isomerization of transstilbene and similar systems, and to simulate the corresponding experimental data. ${ }^{23-29}$ In an adiabatic model, ${ }^{23-25}$ see Fig. 6.1, one assumes the existence of a small barrier on the first excited state $\left(S_{1}\right)$ and fast Intramolecular Vibrational Energy Redistribution (IVR), so that the RRKM model can be used to calculate the reaction rate. ${ }^{66,67}$ The adiabatic model was successfully applied to the description of molecular beam experiments, ${ }^{28,68}$ and an effective barrier height was estimated to be about $1200 \mathrm{~cm}^{-1}$. Nevertheless, the pressure dependence of the reaction rate is not well reproduced and requires the introduction/assumption of "pressure 
dependent barrier", however without physically clear picture. In particular, at low pressures theoretical model fits reasonably well to the experimental data, ${ }^{69,70}$ but at pressures above 5 bar the experimental rate constant levels off at $20-30 \mathrm{~ns}^{-1}$, while the theoretical high-pressure limit ${ }^{70}$ is at $76 \mathrm{~ns}^{-1}$. Alternatively, a non-adiabatic model involving the transition to another excited state was suggested ${ }^{26-29}$ to explain the photoisomerization mechanism. It is supported by the fact that the twisted conformation of stilbene, where the radiationless transition to the ground state takes place, belongs to the distinct electronic state $\left(S_{2}\right)$ characterized as the doubly excited or zwitterionic state. ${ }^{26-29}$

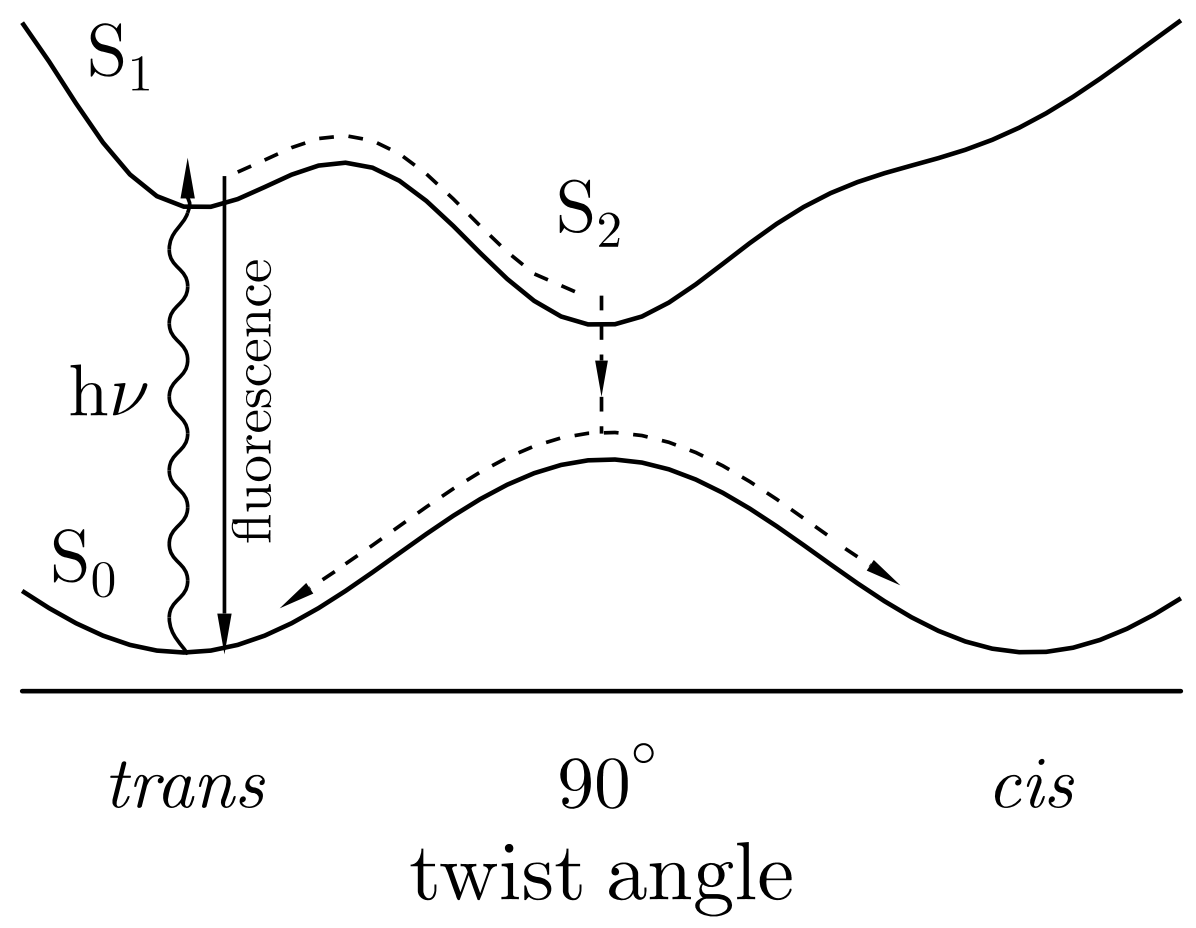

Figure 6.1: Schematic representation of the adiabatic model for the trans-cis photoisomerization of stilbene and stilbene-like molecules. It assumes the existence of a small barrier on the first excited state PES and fast IVR. Activated barrier crossing is assumed to be the rate limiting step, a radiationless transition into the ground state takes place from the twisted conformation, which, nevertheless, belongs to the distinct electron PES. 


\subsection{Basic assumptions of the model}

The origin of a small barrier in the adiabatic model for the twist around the central ethylenic bond, which is also assumed to be the reaction coordinate, is not clear. A bond length is a good qualitative measure of the electron density located on it, and the associated barrier height for the torsional motion around it. The longer the bond length - the lower the barrier, and vice versa. A series of accurate quantum chemical calculations show, that in the ground state of trans-stilbene the central ethylenic bond $\left(C_{7}-C_{8}\right.$ in Fig. 6.2) has the double bond character with the length of about (dependent on the method used) $1.36 / 1.354 / 1.33 \AA{ }^{71-74}$ whereas the neighboring ones $\left(C_{2}-C_{7}, C_{8}-C_{9}\right)$ have more a single bond character with the lengths of about $1.47 / 1.483 / 1.45 \AA{ }^{71-74}$ As a result, the direct twist around the central bond in the ground state has a large activation barrier, while the potential energy surface for the twist of the phenyl rings is flat in the range of about 30 degrees. ${ }^{75,76}$

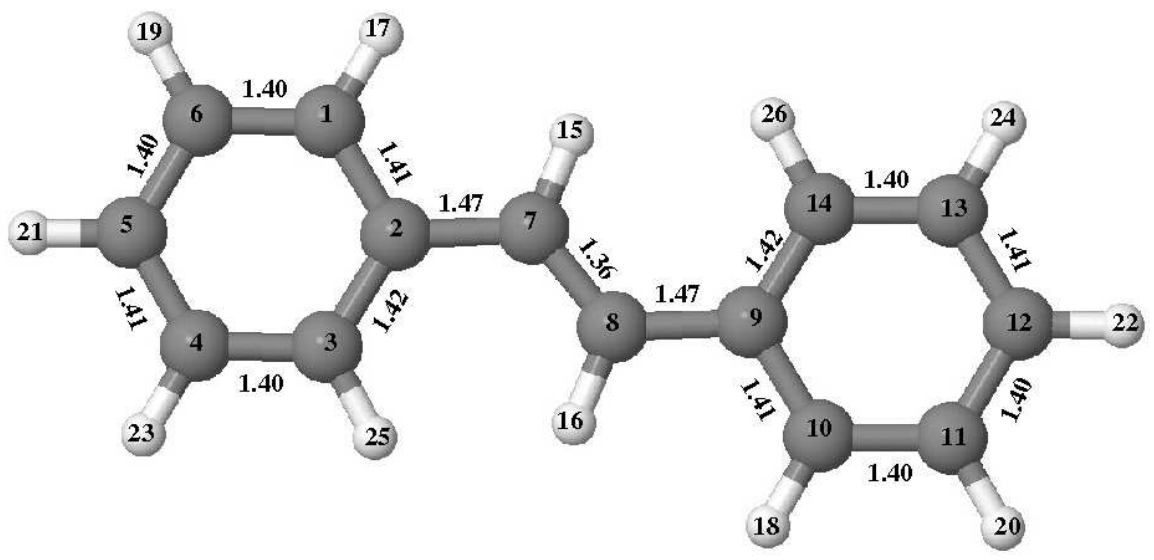

Figure 6.2: Schematic representation of trans-stilbene and their bond lengths for the ground $\left(S_{0}\right)$ state. Carbon atoms are represented by grey balls, whereas the hydrogen atoms by white balls, respectively.

On the other hand, in the first exited state the corresponding lengths were estimated ${ }^{71-74}$ to be $1.40 / 1.432 \AA$ for the central ethylenic bond $\left(C_{7}-C_{8}\right.$ in Fig. 6.3$)$ and $1.41 / 1.419 \AA$ for the neighboring ones $\left(C_{2}-C_{7}, C_{8}-C_{9}\right)$, which is in between the single $(\sim 1.5 \AA)$ and the double 
bond character $(\sim 1.35 \AA)$. As a result, in the first excited state the trans-stilbene is planar, ${ }^{71,77}$ and the twist around the central bond is still expected to have a considerable barrier.

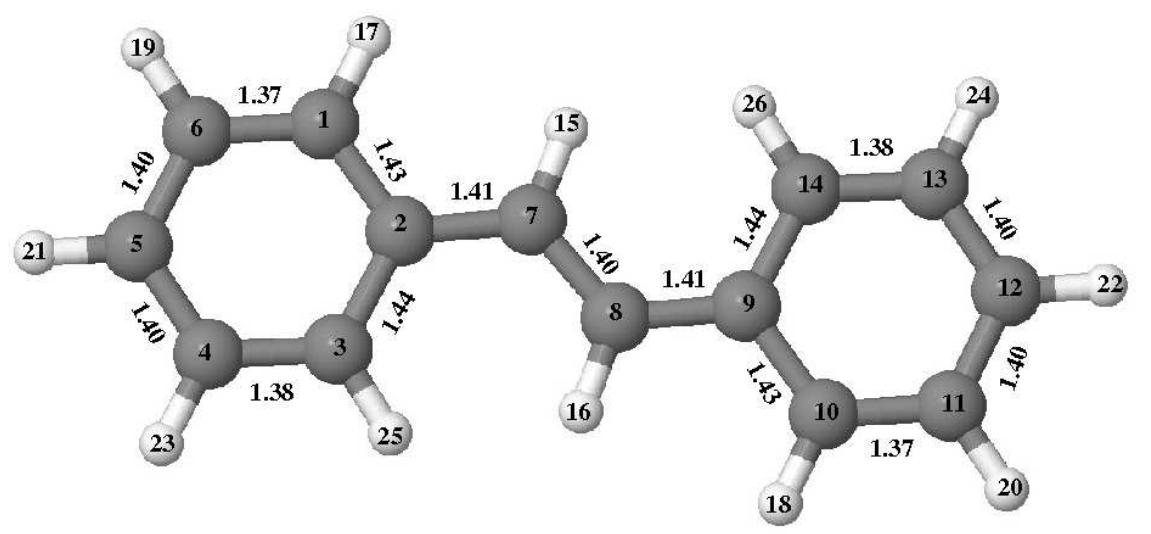

Figure 6.3: Schematic representation of trans-stilbene and their bond lengths for the single $\left(S_{1}\right)$ excited state.

Finally, our quantum-chemical calculations have shown that in the doubly excited state the central bond has the length of $1.45 \AA$, which is close to the single bond character, and, therefore, the twist around it is expected to be nearly the barrierless.

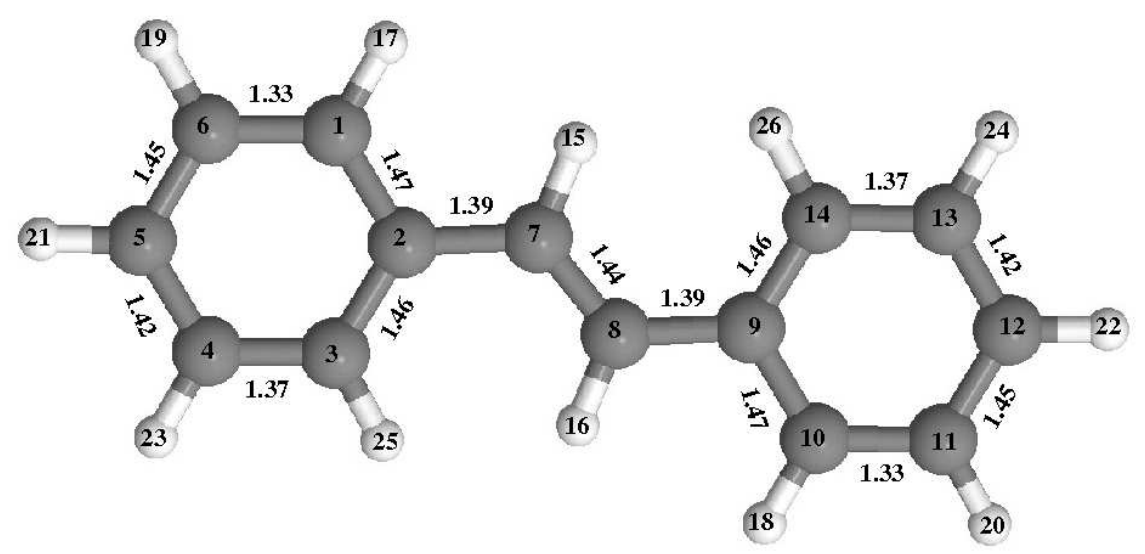

Figure 6.4: Schematic representation of trans-stilbene and their bond lengths for the double $\left(S_{2}\right)$ excited state.

Thus, the non-adiabatic pathway of the photoisomerization, involving the preliminary transition from $S_{1}$ to $S_{2}$ state within the planar geometry of trans-stilbene, may indeed be favored 
over the direct twist around the central bond in the $S_{1}$ state. In this case the twist around the central bond is not considered as the rate limiting step, and the reaction coordinate for the $S_{1} \rightarrow S_{2}$ conversion can be any, including in-plane vibrations. For example, it was found in Ref., ${ }^{28}$ that the non-adiabatic RRKM fits well the experimental data if one uses a normal mode with the frequency of $400 \mathrm{~cm}^{-1}$ as the reaction coordinate, which is obviously not the frequency of the twist around the central bond.

In the present chapter we estimate an efficiency of the non-adiabatic pathway of the photoisomerization based on the following model, consisting of the two multi-dimensional PESs schematically shown on Fig. 6.5. We assume that the molecule in first excited state $S_{1}$ is prepared with a small energy excess (the frequency of the laser pulse is about the $0-0$ transition frequency), so that the energy distribution on $S_{1}$ PES may be considered as the canonical one with the temperature $T$ of the surrounding media, possibly corrected to account for the vibrational cooling effect.

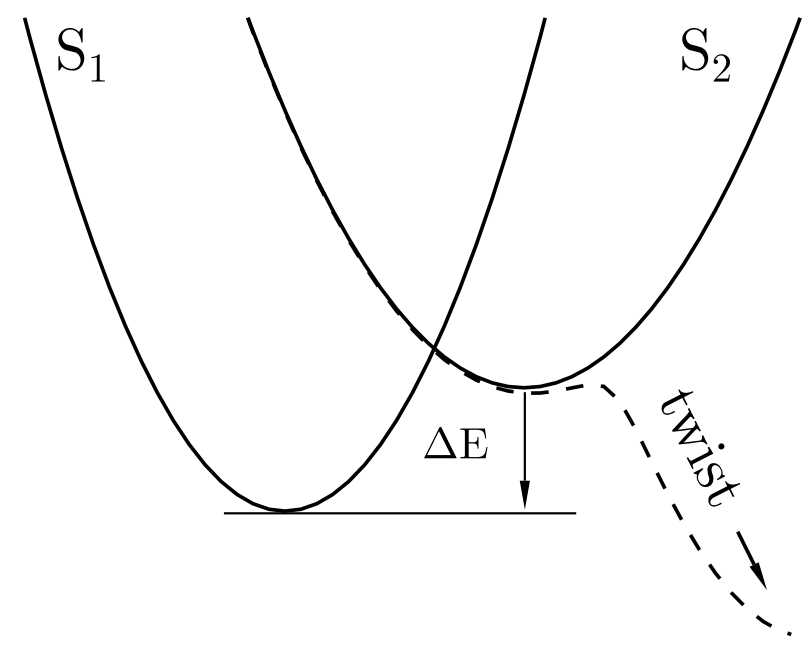

Figure 6.5: Schematic model for the trans-cis non-adiabatic photoisomerization of stilbene and stilbene-like molecules. It assumes fast IVR and the non-adiabatic conversion from the first excited state $\left(S_{1}\right)$ into the double excited one $\left(S_{2}\right)$ near the planar geometry to be the rate limiting step. The subsequent twist around the central bond proceeds along $S_{2}$ PES, where it is considered to be nearly barrierless.

The PESs for the $S_{1}$ and $S_{2}$ states near their planar geometry were obtained in the normal mode approximation by ab initio quantum chemistry methods. This should be a reasonably 
good approximation for all modes except for the twist around the central ethylenic bond in $S_{2}$ state, which is nearly barrierless as it was mentioned above. The IVR in trans-stilbene is fast, compared to the time scale of the photoisomerization, and was estimated to take about 20 ps. ${ }^{78-85}$ This makes the transition $S_{1} \rightarrow S_{2}$ irreversible, since the molecule very fast twists to the transition state with the energy, considerably lower than the minimum of the $S_{1}$ PES. Such a situation is similar to the non-adiabatic transitions in the "inverted region" of the Free-EnergyGap Law, where the transitions are thermally activated but irreversible. However, the normal modes approximation at the local energy minimum of $S_{2}$ PES near the planar geometry was used to restore the energy profile in the transition zone.

The energy splitting between the PESs plays a key role in the non-adiabatic dynamics. In Section 5.5 we estimated the energy gap between the minima of $S_{2}$ and $S_{1}$ states in transstilbene from the spectroscopical data and the quantum-chemical calculations to be about $\Delta E=$ $E_{2}-E_{1}=0.6 \mathrm{eV}$ (the local minimum of $S_{2}$ PES lies above the minimum of $S_{1}$ ). This value is rather large and seems to prevent any surface crossing events, since it requires too large (and rare) fluctuations of the energy per (reaction) degree of freedom. Nevertheless, in the canonical ensemble the $S_{1} \rightarrow S_{2}$ transition are not forbidden energetically, although the mechanism of the nonadiabatic transitions in polyatomic molecules may fundamentally be different from those in small molecules. Within the classical mechanics picture, in the one-dimensional case the energetically allowed nonadiabatic transitions always proceed via the surface crossing events, whereas in the multi-dimensional case the fast IVR redistributes the energy among other degrees of freedom, and may effectively prevent the surface crossing events.

The structure of p-coumaric acid, schematically shown on Figs. 6.6-6.8, is similar to those of trans-stilbene. 


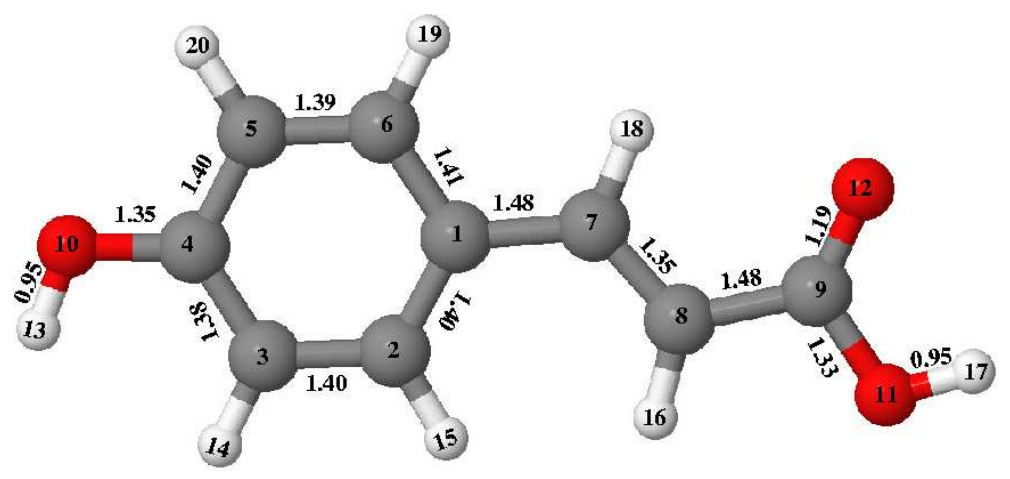

Figure 6.6: Schematic representation of p-coumaric acid and their bond lengths for the ground $\left(S_{0}\right)$ state. Carbon atoms are represented by grey balls, whereas the hydrogen and oxygen atoms by white and red balls, respectively.

Similarly to the trans-stilbene case we have found that the central bond $\left(C_{7}-C_{8}\right.$ in Fig. 6.6) in the ground state of p-coumaric acid has a double bond character of $1.35 \AA$, which means that it has a large barrier preventing the rotation around it. The neighboring bonds $\left(C_{1}-C_{7}, C_{8}-C_{9}\right)$ both have the length of about $1.48 \AA$ which is close to the single-bond character, thus making the corresponding potentials for the twist around them to be flat.

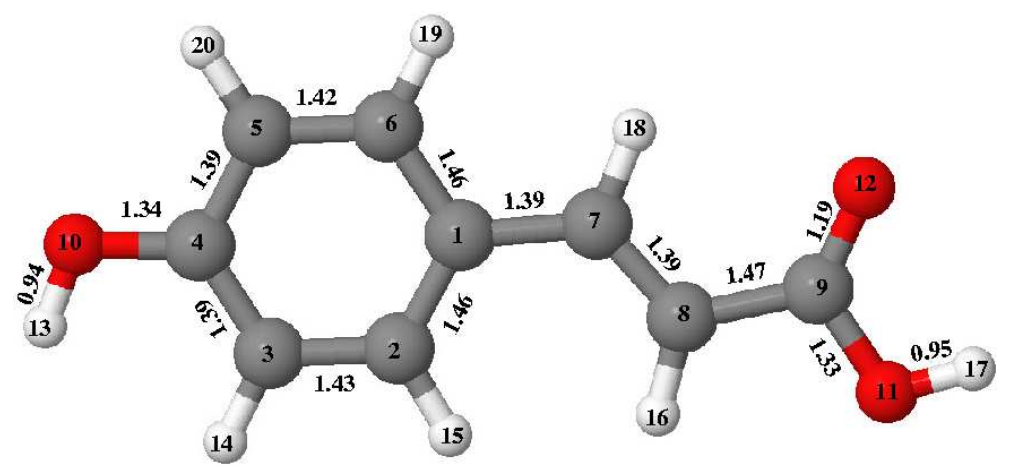

Figure 6.7: Schematic representation of p-coumaric acid and their bond lengths for the single $\left(S_{1}\right)$ excited state.

In the first excited state the length of the central bond and one of the neighboring bonds $\left(C_{1}-C_{7}, C_{7}-C_{8}\right)$ becomes $1.39 \AA$, whereas the bond length $C_{8}-C_{9}$ is just slightly affected and becomes $1.47 \AA$, but the rotation around it does not lead to the isomerization, see Fig. 6.7. 


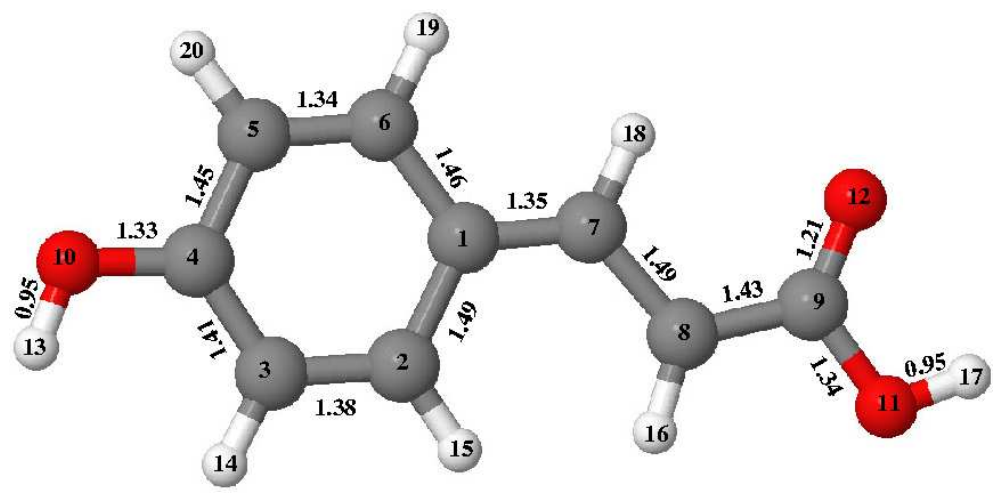

Figure 6.8: Schematic representation of p-coumaric acid and their bond lengths for the double $\left(S_{2}\right)$ excited state.

Finally, in the doubly excited state the central bond has the length of $1.49 \AA$, which is of single bond character, and the twist around it may again become nearly barriereless, see Fig. 6.8. Thus, the quantum chemistry of p-coumaric acid and its photoisomerization mechanism looks quite similar to the trans-stilbene. However, the p-coumaric acid chromophore exhibits much faster isomerization rate of about several picoseconds. ${ }^{86-88}$ We attempt to clarify the origin of this difference below.

\subsection{NQCA and the photoisomerization processes of poly- atomic molecules}

For the case of the two PESs the Hamiltonian of the polyatomic molecule can be written as

$$
\hat{H}=\hat{H}_{N}^{(1)}|1\rangle\left\langle 1\left|+\hat{H}_{N}^{(2)}\right| 2\right\rangle\langle 2|+\hat{V},
$$

where $\hat{H}_{N}^{(1,2)}$ are the PESs Hamiltonians depending on the coordinates of the nuclei, $\hat{V}$ is the Hamiltonian of the coupling between them, and $|n\rangle\langle n|$ are the projectors on the $\mathrm{n}$-th electronic state. 
In the case of the non-adiabatic photoisomerization the Hamiltonian of the coupling

$$
\hat{W}=-\sum_{\alpha=1}^{n} \frac{i \hbar}{m_{\alpha}} \hat{\mathbf{p}}_{\alpha} \cdot \hat{\mathbf{d}}_{\alpha},
$$

is defined by scalar products of the momenta of the atomic centra $\mathbf{p}_{\alpha}$ and the corresponding non-adiabatic coupling vector $\mathbf{d}_{\alpha}$. Here also $\hbar$ is the Planck constant, and $m_{\alpha}$ is the mass of the $\alpha$-th atom in the molecule.

The kinetic equations of quantum-classical approach in the absence of the backward reaction can readily be obtained from those presented in Section (4) by formally taking the limit of infinite negative free energy change in the course of the reaction, and have the following form

$$
\frac{d \sigma(t)}{d t}=-\frac{1}{N} \sum_{k=1}^{N} \operatorname{Im}\left(\chi_{k}\left(q_{k}(t)\right) W_{k}\left(q_{k}(t)\right)\right),
$$

where $\sigma(t)$ is the probability of being on $S_{1}$ PES, $N$ is in practice a large number of the MD trajectories sufficient to sample the phase space of $S_{1}$, and

$$
\frac{d \chi_{k}\left(q_{k}(t)\right)}{d t}=i \Omega_{k}\left(q_{k}(t)\right) \chi_{k}\left(q_{k}(t)\right)+2 i W_{k}\left(q_{k}(t)\right) \sigma(t)
$$

is the auxiliary function for the given trajectory, which accounts for the quantum phase of transition and for the memory effects. The non-adiabatic coupling and the PESs Hamiltonian functions difference $\Omega_{k}\left(q_{k}(t)\right)$ (the vertical energy gap) depending on the corresponding phase space trajectory $q_{k}(t)$ are

$$
W_{k}(t)=-\sum_{\alpha=1}^{n} \frac{i \hbar}{m_{\alpha}} \mathbf{p}_{\alpha}\left(q_{k}(t)\right) \cdot \mathbf{d}_{\alpha}\left(q_{k}(t)\right)
$$

and

$$
\Omega_{k}\left(q_{k}(t)\right)=H_{N}^{(2)}\left(q_{k}(t)\right)-H_{N}^{(1)}\left(q_{k}(t)\right)+\Omega_{0},
$$

where $\Omega_{0}$ is the energy shift between the minima of the first PESs counted for the minima of the second PESs.

The numerical approach to solve Eqs.(6.3), (6.4) parallels those described in Section 4. The normal modes approximation was employed to represent the multidimensional PESs, whereas 
the components of the nonadiabatic coupling vectors $\mathbf{d}_{\alpha}$ were assumed to be constant in the vicinity of the equilibrium geometry of $S_{1}$ PES. Both normal modes and the nonadiabatic coupling vectors were obtained by ab initio quantum-chemical methods, calculation of the vertical energy gap along the trajectories is outlined in Section 5.4.

\subsection{Results - Photoisomerization kinetics of trans-stilbene molecule}

For the simulation of the photoisomerization kinetics of trans-stilbene we used the value of the vertical energy splitting between the minima of the $S_{2}$ and $S_{1}$ states of $0.69 \mathrm{eV}$ (the minimum of $S_{2}$ state lies considerably higher than the minimum of $S_{1}$ ), which is consistent with both spectroscopical data for one- and two- photon absorption spectra of trans-stilbene, see Section 5.5. The potential energy surfaces in the normal mode approximation were obtained using GAMESS US software for ab initio quantum chemical calculations and the CASSCF(10e/10o) approach. The non-adiabatic coupling vector was calculated by freely available COLUMBUS quantum chemistry programs suit on the MR-CI level. We are mainly concentrate on the investigation of the mechanisms of the photoisomerization rather than on an accurate fitting of the available experimental data. Fig. 6.9 shows the simulated kinetics of (irreversible) non-adiabatic transition from $S_{1}$ to $S_{2}$ state, which is also the photoisomerization kinetics in the framework of the considered model assuming non-adiabatic $S_{1} \rightarrow S_{2}$ conversion as the rate limiting step. 


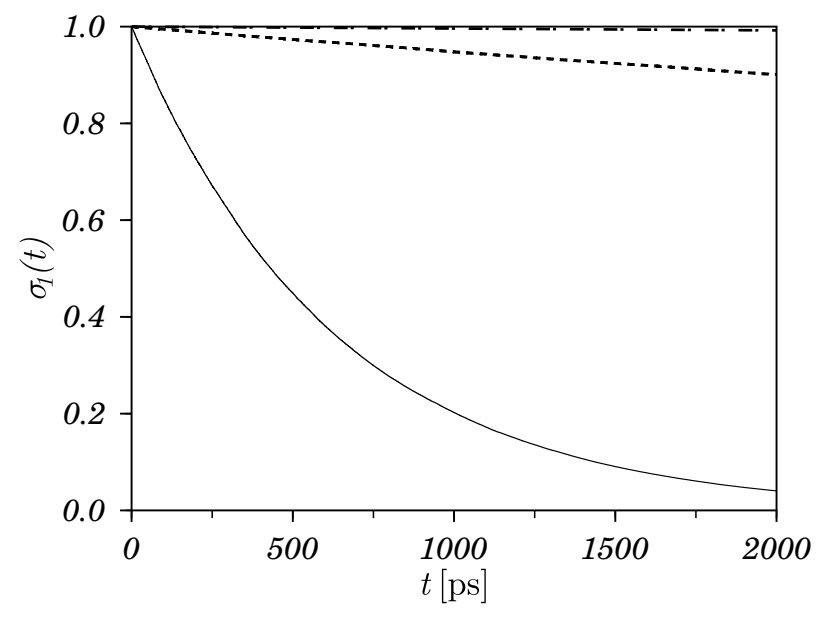

Figure 6.9: The kinetics of the non-adiabatic transition from $S_{1}$ to $S_{2}$ state for the transstilbene, solid line. Dashed line shows the kinetic of the non-adiabatic transition from $S_{1}$ to $S_{2}$ in the absence of the high-frequency normal modes. Dashed-dotted line shows the kinetic of the non-adiabatic transition from $S_{1}$ to $S_{2}$ in the absence of the low and middle frequency normal modes.

The corresponding kinetics is shown on Fig. 6.9. It has a single-exponential character and is well fitted by the formula

$$
\sigma(t)=e^{-t / \tau}, \tau=630 \mathrm{ps}
$$

Note, however, that in our simulation we have neglected by the cooling effect of the reaction. Indeed, the molecules with higher total energies, present in the canonical distribution, should react faster, whereas the molecules with the total energy below some energy threshold may not react at all. Under realistic experimental conditions this leads to the two-exponential decay, with the faster exponent corresponding to the so-called first-order photoisomerization reaction followed by a slower stage when the remaining cold molecules are heated up by the collisions with the bath molecules. Although this cooling effect can be incorporated in our simulation procedure assuming the IVR to be faster than the reaction, which is certainly true for the case of trans-stilbene, we analyze the underlying mechanism of the first-order photoisomerization kinetics without influence of the cooling effect.

The obtained results are quite surprising from the standpoint of the standard models of the 
non-adiabatic transitions, which usually assume the surface crossing events along one or few reaction degrees of freedom. In our simulation the minimum of $S_{2}$ state lies $0.69 \mathrm{eV}$ above the minimum of $S_{1}$ state, and, therefore, the vertical energy splitting between the PESs at the minimum of $S_{1}$ state cannot be smaller than $0.69 \mathrm{eV}$. Therefore, the (thermal) energy fluctuations on a single reaction degree of freedom, which may potentially lead to the surface crossing events, have to be about of this value. For stilbene, being in thermal equilibrium at room temperature with its 72 internal degrees of freedom, these are too rare events to contribute to the reaction. Indeed, Figure 6.10 shows the vertical energy splitting between the $S_{1}$ and $S_{2}$ PESs as the function of time taken along one representative trajectory. The vertical energy splitting fluctuates between $-0.5 \mathrm{eV}$ and $-1.5 \mathrm{eV}$ but does not exhibit surface crossing events, when the vertical energy splitting passes through the zero mark.

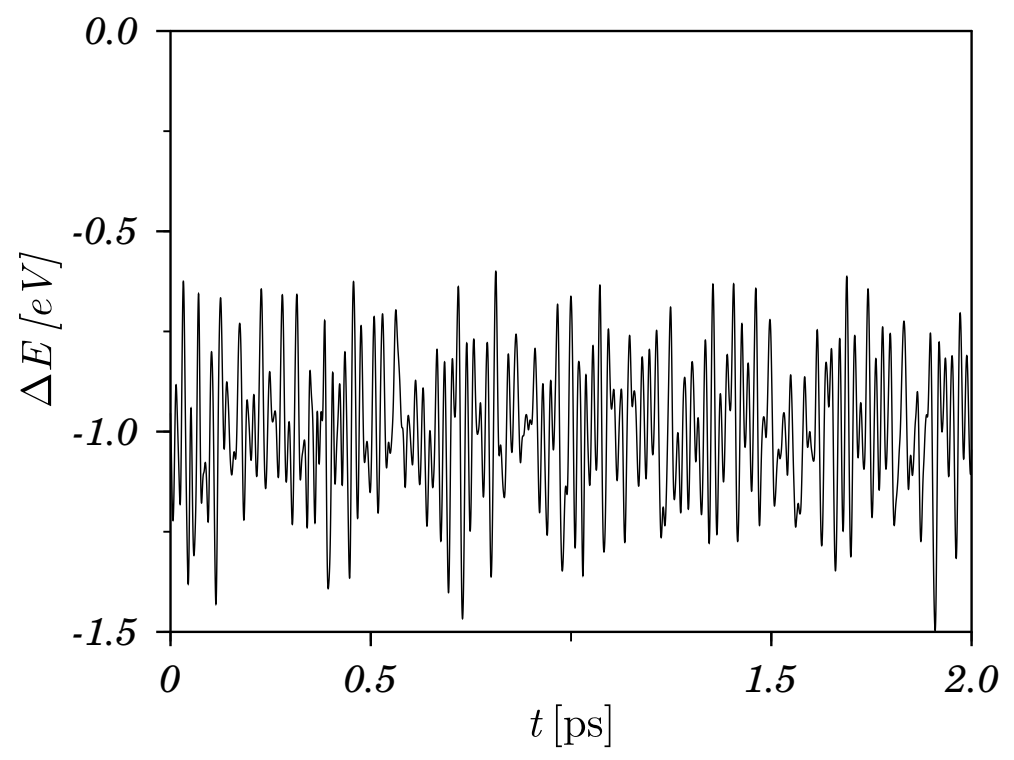

Figure 6.10: Time dependence of the vertical energy splitting, for trans-stilbene, along a single representative trajectory on $S_{1}$ PES. It is seen that the surface crossing events are absent ( they correspond to the crossing $\Delta E=0$ level).

To determine normal modes being most active in the non-adiabatic coupling between the PESs, we have plotted an absolute value of the Fourier transform of $W_{k}(t)$ defined in Eq. (6.5), averaged over many trajectories. This gives us the information about the frequency of the cor- 
responding normal mode and the strength of its contribution into the coupling, see Figure 6.11. The distinct frequency domains are clearly seen in Figure 6.11. In the low-frequency domain with mainly torsional, and bending motion of the molecule, up to $\sim 500 \mathrm{~cm}^{-1}$, one has only one line with a relatively small amplitude. The mid-frequency domain from $\sim 500 \mathrm{~cm}^{-1}$ to $\sim 2000 \mathrm{~cm}^{-1}$, contains a number of lines with different amplitudes. Finally, the high-frequency domain involves normal modes with the dominant contribution of $C-H$ stretch vibrations located around $3300 \mathrm{~cm}^{-1}$. The lines in this region are the most intense. In particular this is due to larger velocities of the high frequency modes, see also Eq. (6.5).

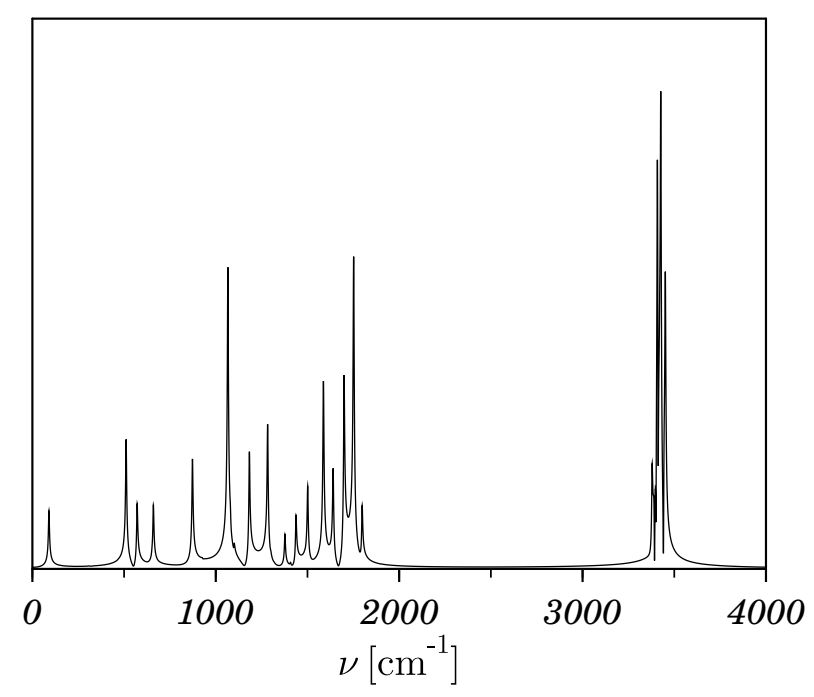

Figure 6.11: Absolute value of the Fourier transform of the non-adiabatic couplings $\mathrm{W}$ for trans-stilbene, averaged over many MD trajectories. Three frequency domains can roughly be defined as the low frequency $\left(0-500 \mathrm{~cm}^{-1}\right)$, the middle frequency $\left(500-2000 \mathrm{~cm}^{-1}\right)$, the high frequency $\left(3300-4000 \mathrm{~cm}^{-1}\right)$ domains.

The dynamics of high frequency modes is often neglected in ab initio simulations of the structural changes of polyatomic molecules, by constraining some bond lengths/angles to their equilibrium values. In the systems with slow reaction modes the above procedure allows to increase the simulation time step considerably, and, as the result, to achieve a longer time scale of the simulation and/or to reduce the computational cost. In order to study the importance of the high-frequency modes for the model under consideration, we have also simulated the 
dynamics of the non-adiabatic transitions in the absence of the high-frequency vibrations of the stilbene molecule, and have set the initial energy of the corresponding normal mode oscillators to zero when sampling from the thermal distribution. The corresponding kinetics is shown on Figure 6.9 by the dashed line. It is readily seen that, when removing the high-frequency modes the reaction rate is reduced by a factor of 30 , giving the characteristic decay time of about $20 \mathrm{~ns}$. On the other hand, when freezing the low and the middle frequency normal modes and keeping only the high-frequency vibrations of the stilbene molecule, we obtain even a slower reaction with the decay time $>200$ ns.

\subsection{Results - Photoisomerization kinetics of p-coumaric acid molecule}

To perform ab initio simulations for the case of p-coumaric acid within the frameworks of the suggested model, we have to estimate the energy difference between the minima of $S_{2}$ and $S_{1}$ PESs, and we use the same approach as for trans-stilbene, see Section 5.5. It gives, however, a qualitatively different situation, the minimum of $S_{2}$ PES lies below the minimum of the $S_{1}$ PES by $0.69 \mathrm{eV}$, which results in the frequent surface crossing events, and, as the result, in the much faster photoisomerization, see below.

The simulated kinetics of $S_{1} \rightarrow S_{2}$ transition is shown on Fig. 6.12, and is indeed very fast compared to the stilbene kinetics, see Eq. (6.7). The decay has one-exponential character and is well fitted by the formula

$$
\sigma(t)=e^{-t / \tau}, \tau=0.44 \mathrm{ps}
$$




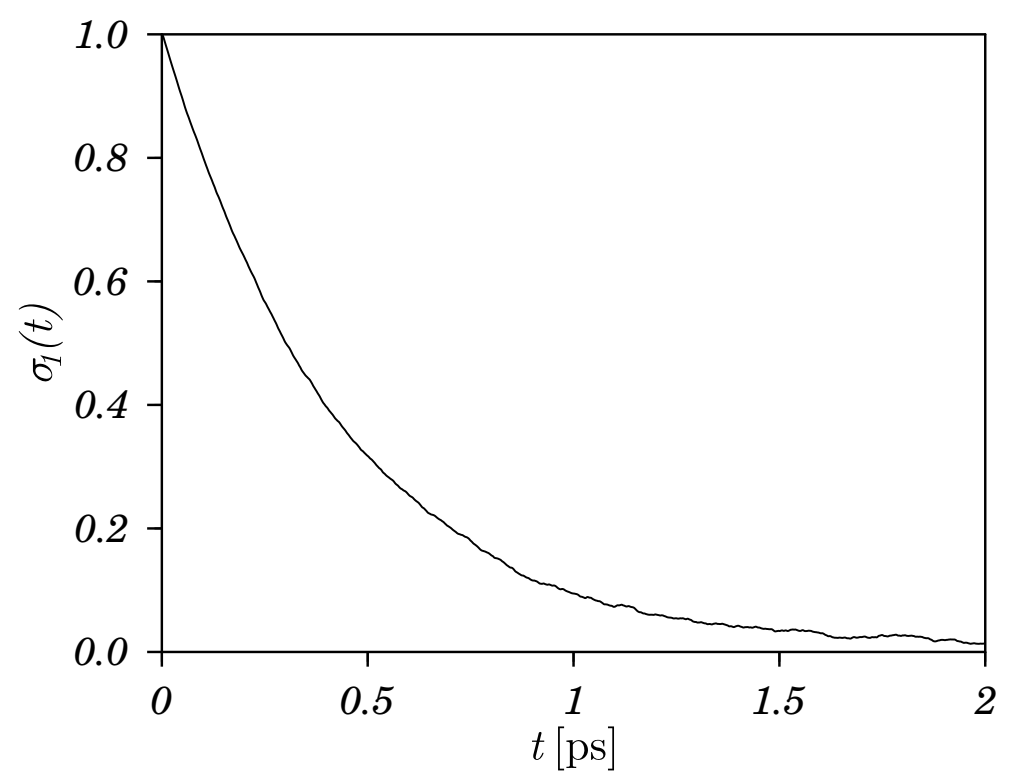

Figure 6.12: The kinetics of the non-adiabatic transition from $S_{1}$ to $S_{2}$ state for p-coumaric acid. The following set of parameters was used for the simulation : $N=1000, T=270$, where $N$ is the number of MD trajectories and $T$ is the temperature corespondly

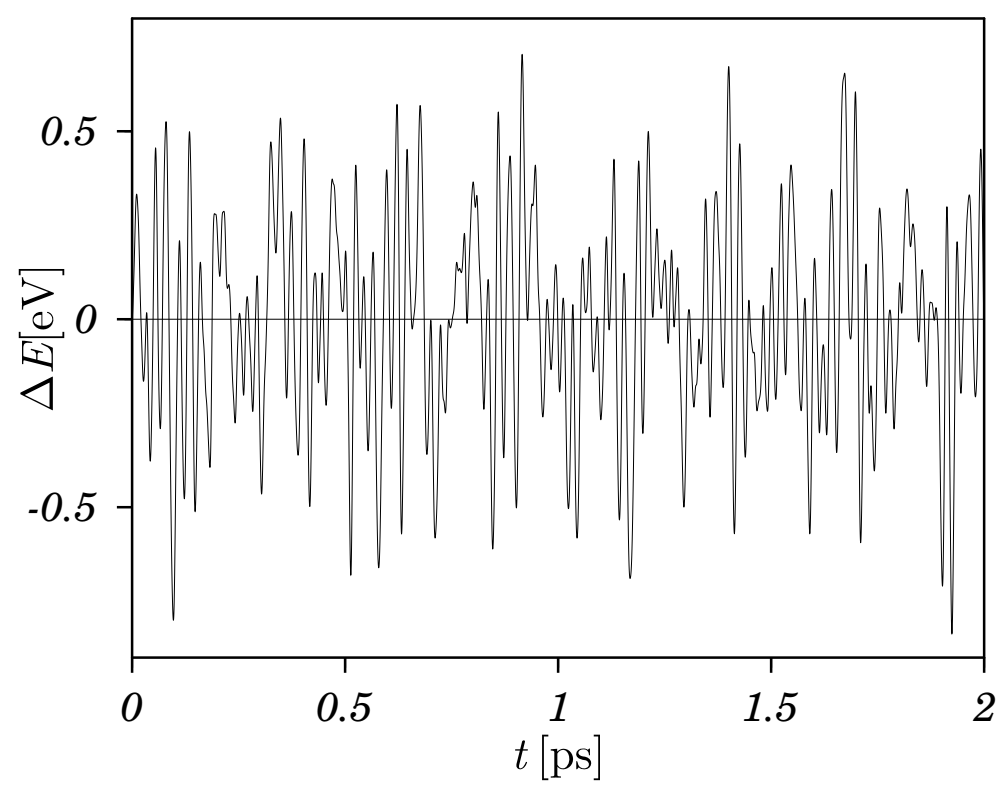

Figure 6.13: Time dependence of the vertical energy splitting for p-coumaric acid, along a single representative trajectory on $S_{1}$ PES and it can be observed that exhibit surface crossing events (they correspond to the crossing of $\Delta E=0$ level). 
The reason for the drastic acceleration of the photoisomerization kinetics becomes clear from Fig. 6.13, where the energy difference between the PESs along some representative trajectory is plotted. Change of the sign of $\Delta E$ indicates the surface crossing event, and there are many of them on the time scale of the reaction. Thus one may conclude, that the dominant mechanism of the non-adiabatic transitions in p-coumaric acid is via surface crossing events, which explains its much faster isomerization than for stilbene.

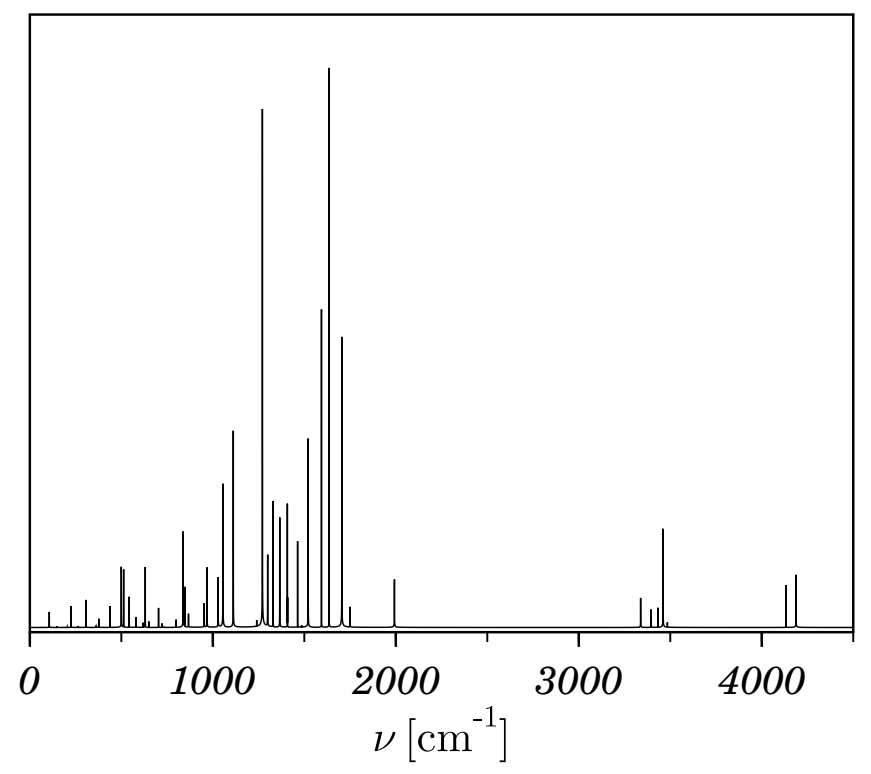

Figure 6.14: Absolute value of the Fourier transform of the non-adiabatic couplings W, for pcoumaric acid, averaged over many MD trajectories. Three frequency domains can be roughly defied : the low frequency $\left(0-1000 \mathrm{~cm}^{-1}\right)$, the middle frequency $\left(1000-2000 \mathrm{~cm}^{-1}\right)$, the high frequency $\left(3300-4200 \mathrm{~cm}^{-1}\right)$ domains.

The high-frequency domain in the non-adiabatic coupling is not well pronounced, see Fig. 6.14, and removing frequencies higher than $3000 \mathrm{~cm}^{-1}$ from the calculations does not considerably affect the kinetics. 


\subsection{Conclusion}

We have carried out ab initio simulations of the trans-cis photoisomerization kinetics of the two polyatomic molecules, stilbene and p-coumaric acid chromophore. The isomerization after photo-excitation into the first excited state was modeled as a two-stage process, with the ratelimiting step to be the non-adiabatic conversion from a single into a double excited state near the planar geometry of the corresponding trans-isomers. The actual isomerization was assumed to take place in the double excited state, where it is nearly barrierless and fast. All parameters of the model were estimated either from high-level quantum-chemical methods (equilibrium geometries, normal modes and their frequencies, non-adiabatic coupling vectors) or from spectroscopical data. The latter were used to obtain the accurate relative energies of the electron states involved in the simulations. The time scale of the photoisomerization kinetics was well reproduced. For gas-phase stilbene at room temperature the non-adiabatic pathway gives a characteristic time of approximately $630 \mathrm{ps,} \mathrm{whereas} \mathrm{p-coumaric} \mathrm{acid} \mathrm{photoisomerization} \mathrm{un-}$ der same conditions is predicted to be faster than $1 \mathrm{ps}$. A deep insight into the mechanism of the reaction reveals, that in the case of stilbene the non-adiabatic conversion between the single and the double excited states proceeds without surface crossing events, via relaxation-like mechanism, when the internal degrees of freedom play the role of the bath. On the other hand, the relative energetics of the single and the double excited states in p-coumaric acid is opposite, which leads to a frequent surface crossing events on the time scale of the reaction and, as the result, to a much faster photoisomerization. 


\section{A}

\section{APPENDIX: Input description GAMESS}

\section{US software}

This section describes the input data of GAMESS US programs used in our simulations.

CONTRL group $=$ this group specifies the type of wavefunction, the type of calculation, use of core potentials and similar fundamental job control options.

SCFTYP $=$ specifies self-consistent field wavefunction.

MCSCF $=$ Multiconfigurational self-consistent field wavefunction.

RUNTYP $=$ specifies the type of computation, for example at a single geometry point :

$=$ OPTIMIZE optimize the molecular geometry using analytic energy gradients.

$=$ HESSIAN molecular energy plus gradient plus second derivatives, including harmonic vibrational analysis.

$=$ ENERGY molecular energy.

$=$ TRANSITN compute transition dipole moment.

$\mathbf{E X E T Y P}=\mathbf{R U N}$ actually do the run.

MAXIT $=$ maximum number of SCF iteration cycles.

COORD $=$ CART cartesian coordinates will be input. 
CISTEP $=$ GUGA this group is the control box for Graphical Unitary Group Approach (GUGA) CI calculations or determinant based CI.

$\mathbf{D H}=$ double zeta basis set

NDFUNC $=$ number of heavy atom polarization function to be used.

POLAR $=$ DUNNING exponent of polarization function.

GUESS $=$ MOREAD read in formatted vectors punched by an earlier run. This requires a VEC group and you must to pay attention to NORB below.

NORB $=$ the number of orbitals to be read in the VEC group.

VEC group $=$ this group consists of formatted vectors, as written onto an output file in a previous run.

$\mathbf{C 1}=$ the group is set to $\mathrm{C} 1$ (no symmetry used).

FORS $=$.T. flag specifying the Full Optimized Reaction Space set of configuration should be generated. This is usually set true for MCSCF runs.

NMCC $=$ number of MCSCF core molecular orbitals (MOs).

NDOC $=$ number of doubly occupied MOs in the reference.

NVAL $=$ number of empty MOs in the reference.

GUGDIA group $=$ this group provides control over the Davidson method diagonalization step.

NSTATE $=$ number of CI states to be found.

ITERMX = maximum number of iterations

GUGDM group $=$ this group provides further control over formation of the one electron density.

NFLGDM $=$ controls each state's density formation, $0 \rightarrow$ do not form density for this state, $1 \rightarrow$ form density and naturals orbitals for this state.

IROOT $=$ the root whose density matrix is saved on desk for later computation of properties. You may save only one state's density per run, by default this is the ground state (default 
$=1)$.

GUGDM2 group $=$ this group provides control over formation of the 2-particle density matrix.

WSTATE $=$ the default is to optimize a pure ground state.

STATPT group $=$ this group controls the search for stationary points.

NSTEP $=$ maximum number of steps to take.

DXMAX = initial trust radius of the step, in Bohr.

LAGRAN group $=$ this group provides further control over formation of CI Lagrangian, a quantity which is necessary for the computation of CI gradients.

NDAR = number of direct access logical record to be used for the integral sort.

FORCE group $=$ this group controls the computation of the hessian matrix and an optimal harmonic vibrational analysis.

PURIFY = the hessian and dipole derivative tensor can be purified by transformation from Cartesian to internal coordinates and back to Cartesian coordinates. This effectively zeros the frequencies of the translation and rotation modes, along with their IR intensities. The purified quantities are written on an output file. 


\section{B}

\section{APPENDIX: Energy and structure}

\section{parameters calculations of ground state}

\section{for p-coumaric acid molecule}

In this chapter the energy and the structure parameters calculations of the ground state of pcoumaric acid will be given. These calculations have been done using the GAMESS US software. Subsequently the program structure for optimizing the system, for calculating the normal modes (via calculating the Hessian matrix) and for the calculation of the energy values of the ground state are given . For calculating all this parameters we have used the Multi Configuration Self Consistent Field method (MCSCF). 


\section{B.1 Optimize the ground state for p-coumaric acid}

\$CONTRL SCFTYP=MCSCF RUNTYP=OPTIMIZE EXETYP=RUN MAXIT=100 COORD $=$ CART $\$ E N D$

$\$ M C S C F$ CISTEP $=$ GUGA MAXIT=200 \$END

$\$ S Y S T E M$ MEMORY=100000000 TIMLIM=100000 \$END

\$BASIS GBASIS=DH NDFUNC $=1$ POLAR=DUNNING \$END

\$GUESS GUESS=MOREAD NORB=196 \$END

$\$$ DRT GROUP $=$ C1 FORS $=$.T. $\mathrm{NMCC}=38 \mathrm{NDOC}=5 \mathrm{NVAL}=3$ \$END

\$GUGDIA NSTATE $=6$ ITERMX $=200$ \$END

\$GUGDM NFLGDM(1) $=1,0,0,0,0$ IROOT $=1$ \$END

\$GUGDM2 WSTATE $(1)=1.0,0.0,0.0$ \$END

$\$$ STATPT NSTEP $=100$ DXMAX $=0.05$ \$END

\$DATA

CNH 1

$\begin{array}{lrrrr}\mathrm{C} & 6.0 & 0.3857684051 & 0.3208999453 & 0.0000000000 \\ \mathrm{C} & 6.0 & 0.9189011047 & -1.0705721303 & 0.0000000000 \\ \mathrm{C} & 6.0 & 2.2821791178 & -1.2703519400 & 0.0000000000 \\ \mathrm{C} & 6.0 & 3.1581421065 & -0.1627619802 & 0.0000000000 \\ \mathrm{C} & 6.0 & 2.6763870787 & 1.2026049709 & 0.0000000000 \\ \mathrm{C} & 6.0 & 1.3489316597 & 1.4174548998 & 0.0000000000 \\ \mathrm{C} & 6.0 & -0.9495508560 & 0.5487417286 & 0.0000000000 \\ \mathrm{C} & 6.0 & -2.0188598910 & -0.4944398517 & 0.0000000000 \\ \mathrm{C} & 6.0 & -3.3840650816 & -0.0585298875 & 0.0000000000 \\ \mathrm{O} & 8.0 & 4.4846074717 & -0.3008038006 & 0.0000000000 \\ \mathrm{O} & 8.0 & -4.2718031733 & -1.0677972721 & 0.0000000000 \\ \mathrm{O} & 8.0 & -3.7614996214 & 1.0924560514 & 0.0000000000 \\ \mathrm{H} & 1.0 & 4.7426533069 & -1.2131688007 & 0.0000000000 \\ \mathrm{H} & 1.0 & 2.6924726469 & -2.2661664687 & 0.0000000000 \\ \mathrm{H} & 1.0 & 0.2491573923 & -1.9038093823 & 0.0000000000 \\ \mathrm{H} & 1.0 & -1.8048499967 & -1.5412924829 & 0.0000000000 \\ \mathrm{H} & 1.0 & -5.1363756758 & -0.6739316627 & 0.0000000000 \\ \mathrm{H} & 1.0 & -1.3114458701 & 1.5615393684 & 0.0000000000 \\ \mathrm{H} & 1.0 & 0.9664943095 & 2.4227448743 & 0.0000000000 \\ \mathrm{H} & 1.0 & 3.3965770326 & 1.9981162128 & 0.0000000000 \\ \text { SEND } & & & & \end{array}$




\section{\$VEC}

1 1-5.34936986E-06-2.59054439E-05 4.37525072E-04 7.34983648E-06-1.29202763E-05

$120.00000000 \mathrm{E}+005.77913946 \mathrm{E}-05$ 8.40388037E-04 0.00000000E+00-1.09911163E-06

1 3-1.40838966E-05-8.83000701E-06 2.58739131E-06 0.00000000E+00 0.00000000E+00

1 4-2.55280796E-06-7.83602766E-06 4.33781096E-04 1.73162492E-05 6.19662657E-06

$150.00000000 \mathrm{E}+00-4.87566158 \mathrm{E}-04-6.82310236 \mathrm{E}-050.00000000 \mathrm{E}+00-1.55967639 \mathrm{E}-05$

16 7.38705060E-07-2.56353495E-07-3.45157617E-06 0.00000000E+00 0.00000000E+00

$179.00970631 \mathrm{E}-06$ 1.98388096E-07-6.62008741E-04-1.76509596E-05-1.41118283E-05

$180.00000000 \mathrm{E}+00-2.15406823 \mathrm{E}-04-1.26933319 \mathrm{E}-040.00000000 \mathrm{E}+004.12682983 \mathrm{E}-05$

$193.97382599 \mathrm{E}-05-7.64269861 \mathrm{E}-07$ 1.07809154E-05 0.00000000E $+000.00000000 \mathrm{E}+00$

1 10-3.12761130E-05 2.11869318E-04-1.47929846E-04 8.78987051E-06 1.40086759E-06

$1110.00000000 \mathrm{E}+00-7.34033733 \mathrm{E}-04-1.13142354 \mathrm{E}-03$ 0.00000000E+00-2.40558050E-04

1 12-8.84184551E-05-2.61067893E-05 4.88551336E-05 0.00000000E+00 0.00000000E+00

1 13-7.34669106E-06-5.04971100E-05 5.88585224E-04 1.92685167E-06-2.56728121E-05

$1140.00000000 \mathrm{E}+00$ 3.89193093E-04-3.62742971E-04 0.00000000E+00-1.29561814E-05

115 1.04869775E-05-1.88950873E-05 2.56027883E-06 0.00000000E+00 0.00000000E+00 116 5.68371998E-06 3.69798451E-05-4.41778005E-04-2.61928189E-06 1.50285770E-05

$1170.00000000 \mathrm{E}+00$ 4.20986372E-04 2.43212939E-04 0.00000000E+00 1.67007335E-06 $1184.33539282 \mathrm{E}-07$ 1.48415578E-05 4.38441592E-06 0.00000000E+00 0.00000000E+00 119 2.23269994E-06 1.85121526E-05-2.82577107E-04-1.05022784E-05-4.95948800E-06 $1200.00000000 \mathrm{E}+00-1.02149472 \mathrm{E}-04-2.96290910 \mathrm{E}-040.00000000 \mathrm{E}+00-2.39751213 \mathrm{E}-06$ 121 3.49238496E-06 7.37382908E-06-5.89438236E-06 0.00000000E+00 0.00000000E+00 122 6.83019640E-07 1.28143502E-06-7.48293777E-05 2.18127137E-06-1.28018678E-06 123 0.00000000E+00-8.46359856E-05 1.38986091E-04 0.00000000E+00 6.39471133E-06 124 3.09825272E-06 7.89486143E-07 2.46482141E-06 0.00000000E+00 0.00000000E+00 $1252.87695520 \mathrm{E}-06$ 1.22776583E-05-1.57286530E-04-7.68444876E-07-5.57364110E-08 $1260.00000000 \mathrm{E}+00-5.25722138 \mathrm{E}-05-4.71780502 \mathrm{E}-050.00000000 \mathrm{E}+00$ 2.20522232E-06 127 4.43070034E-06 7.28634730E-06-8.58618957E-07 0.00000000E+00 0.00000000E+00 128 9.99866682E-01-2.92060915E-05 4.75162407E-04-9.33886148E-04-9.93480447E-04 $1290.00000000 \mathrm{E}+00$ 2.11390021E-04 8.02971104E-04 0.00000000E+00 6.72202665E-04 130 5.61369037E-04 6.31513146E-04 1.72102941E-05 0.00000000E+00 0.00000000E+00 1 31-1.10460377E-07-3.36220747E-07 1.69699456E-06-3.59052885E-07 1.30187815E-07 $1320.00000000 \mathrm{E}+00$ 9.07012462E-06 7.86449031E-06 0.00000000E+00 7.81914434E-07 133 8.23228987E-08-1.45421053E-06 9.64343888E-07 0.00000000E+00 0.00000000E+00 $134-1.32013342 \mathrm{E}-06-6.56445456 \mathrm{E}-06$ 4.63383251E-05 6.90447237E-07-4.16541862E-07 $1350.00000000 \mathrm{E}+002.89723296 \mathrm{E}-07-7.07085997 \mathrm{E}-060.00000000 \mathrm{E}+00-5.02897308 \mathrm{E}-06$ 1 36-4.11081140E-06-1.88211780E-06 3.96443368E-07 0.00000000E+00 0.00000000E+00 137 1.53152135E-04 2.50165394E-04 3.17519577E-06-2.62292204E-05 1.62148210E-06 1 38-2.88489765E-04-3.28615401E-06 9.45224933E-05 1.37399451E-06-1.06215671E-05 139 1.08234631E-06 1.53273696E-04 1.61468488E-05 1.20696170E-05-6.24087895E-06 1 40-1.94741424E-05

SEND 


\title{
B.2 Hessian calculations for the ground state
}

\author{
$\$ C O N T R L$ SCFTYP=MCSCF RUNTYP=Hessian EXETYP=RUN MAXIT $=100$ COORD=CART \\ \$END \\ \$MCSCF CISTEP $=$ GUGA MAXIT=200 \$END \\ \$SYSTEM MEMORY=100000000 TIMLIM=100000 \$END \\ $\$ B A S I S$ GBASIS $=$ DH NDFUNC $=1$ POLAR $=$ DUNNING $\$ E N D$ \\ \$GUESS GUESS $=$ MOREAD NORB $=196$ \$END \\ $\$ D R T$ GROUP $=\mathrm{C} 1$ FORS $=$. T. $\mathrm{NMCC}=38 \mathrm{NDOC}=5 \mathrm{NVAL}=3$ \$END \\ \$GUGDIA NSTATE $=6$ ITERMX $=200$ \$END \\ $\$$ GUGDM NFLGDM(1)=1,0,0,0,0 IROOT=1 \$END \\ \$GUGDM2 WSTATE(1)=1.0,0.0,0.0,0.0 \$END \\ $\$ S T A T P T$ NSTEP $=100$ DXMAX $=0.05$ \$END \\ \$DATA
}

\section{$\mathrm{CNH} 1$}

$\begin{array}{lllll}\mathrm{C} & \quad 6.0 & 0.4163908690 & 0.2326604093 & 0.0000000000\end{array}$

$\begin{array}{lllll}\mathrm{C} & 6.0 & 0.9667952878 & -1.0537230967 & 0.0000000000\end{array}$

$\begin{array}{lllll}\mathrm{C} & 6.0 & 2.3507625055 & -1.2453757682 & 0.0000000000\end{array}$

$\begin{array}{llllll}\mathrm{C} & 6.0 & 3.2063828898 & -0.1580262437 & 0.0000000000\end{array}$

$\begin{array}{llllll}\mathrm{C} & 6.0 & 2.6832774224 & 1.1383276474 & 0.0000000000\end{array}$

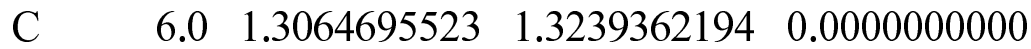

$\begin{array}{lllll}\text { C } & 6.0 & -1.0349985450 & 0.5002690085 & 0.0000000000\end{array}$

$\begin{array}{lllll}\text { C } & 6.0 & -2.0316980051 & -0.4086321816 & 0.0000000000\end{array}$

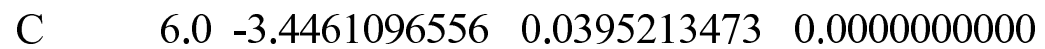

$\begin{array}{llllll}\mathrm{O} & 8.0 & 4.5530882951 & -0.2829549599 & 0.0000000000\end{array}$

$\begin{array}{llllll}\text { O } & 8.0 & -4.2906324743 & -0.9914256329 & 0.0000000000\end{array}$

$\begin{array}{lllll}\mathrm{O} & 8.0 & -3.8252672732 & 1.1707562683 & 0.0000000000\end{array}$

$\mathrm{H} \quad \begin{array}{llllll}1.0 & 4.8068995248 & -1.1948499186 & 0.0000000000\end{array}$

$\mathrm{H} \quad \begin{array}{lllll}\mathrm{H} & 1.0 & 2.7494932557 & -2.2465680163 & 0.0000000000\end{array}$

$\mathrm{H} \quad \begin{array}{lllll}1.0 & 0.3327298818 & -1.9208178077 & 0.0000000000\end{array}$

$\mathrm{H} \quad \begin{array}{lllll} & 1.0 & -1.8690888247 & -1.4693394788 & 0.0000000000\end{array}$

$\mathrm{H} \quad \begin{array}{lllll} & 1.0 & -5.1766588746 & -0.6444362772 & 0.0000000000\end{array}$

$\mathrm{H} \quad \begin{array}{lllll}\mathrm{H} & 1.0 & -1.3238705391 & 1.5366726209 & 0.0000000000\end{array}$

$\begin{array}{llllll}\mathrm{H} & 1.0 & 0.9158711190 & 2.3263321660 & 0.0000000000\end{array}$

$\begin{array}{lllll}\mathrm{H} & 1.0 & 3.3591143628 & 1.9735545752 & 0.0000000000\end{array}$

\$END 


\section{\$VEC}

1 1-5.74241546E-06-2.66200965E-05 4.43162619E-04 6.53775727E-06-1.27549490E-05

$120.00000000 \mathrm{E}+006.36777468 \mathrm{E}-05$ 8.43559247E-04 0.00000000E+00-1.44936503E-06

1 3-1.44709527E-05-8.84764643E-06 2.59455122E-06 0.00000000E+00 0.00000000E+00

1 4-2.70395199E-06-8.44013234E-06 4.35050407E-04 1.63089414E-05 5.07357965E-06

$150.00000000 \mathrm{E}+00-4.91949359 \mathrm{E}-04-6.90824388 \mathrm{E}-050.00000000 \mathrm{E}+00-1.56509379 \mathrm{E}-05$

16 9.71581000E-07-7.17051313E-08-3.42337309E-06 0.00000000E+00 0.00000000E+00

17 8.99142908E-06 4.61815722E-07-6.64216422E-04-1.65539169E-05-1.58923874E-05

$180.00000000 \mathrm{E}+00-2.13328362 \mathrm{E}-04-1.26913909 \mathrm{E}-040.00000000 \mathrm{E}+004.11488616 \mathrm{E}-05$

$193.89178657 \mathrm{E}-05-8.84622402 \mathrm{E}-07$ 1.05841921E-05 0.00000000E $+000.00000000 \mathrm{E}+00$

1 10-3.03409829E-05 2.13240688E-04-1.68660084E-04 1.16820033E-05 4.25030490E-07

$1110.00000000 \mathrm{E}+00-7.54699763 \mathrm{E}-04-1.13369523 \mathrm{E}-03$ 0.00000000E+00-2.34113398E-04

1 12-8.59849466E-05-2.88608913E-05 4.86457109E-05 0.00000000E+00 0.00000000E+00

1 13-7.57993948E-06-5.03436691E-05 5.89171462E-04 3.77727093E-06-2.57647341E-05

$1140.00000000 \mathrm{E}+003.96460058 \mathrm{E}-04-3.69489200 \mathrm{E}-040.00000000 \mathrm{E}+00-1.28726388 \mathrm{E}-05$

115 9.94013808E-06-1.91893094E-05 2.55661387E-06 0.00000000E+00 0.00000000E+00

116 5.34356427E-06 3.58481752E-05-4.39086330E-04-4.07201758E-06 1.61612011E-05

$1170.00000000 \mathrm{E}+00$ 4.25860925E-04 2.46714595E-04 0.00000000E+00 1.42952089E-06

118 4.16124916E-07 1.49514264E-05 4.43499709E-06 0.00000000E+00 0.00000000E+00

1 19 3.06023017E-06 1.70904338E-05-2.84938832E-04-9.09301732E-06-5.79409850E-06

$1200.00000000 \mathrm{E}+00-1.01885552 \mathrm{E}-04-2.97158342 \mathrm{E}-040.00000000 \mathrm{E}+00-2.40642669 \mathrm{E}-06$

121 3.31660701E-06 7.36769003E-06-5.91733780E-06 0.00000000E+00 0.00000000E+00

122 9.72089168E-07 8.19045045E-07-7.42411463E-05 1.70203048E-06-2.21183944E-06

123 0.00000000E+00-8.52962573E-05 1.42079724E-04 0.00000000E+00 6.47132287E-06

124 3.04819364E-06 7.13751466E-07 2.37669079E-06 0.00000000E+00 0.00000000E+00

125 2.91189040E-06 1.24524140E-05-1.59508949E-04-7.94965594E-07-2.23814707E-08

$1260.00000000 \mathrm{E}+00-5.33905628 \mathrm{E}-05-4.75221528 \mathrm{E}-05$ 0.00000000E+00 2.18769934E-06

127 4.47743449E-06 7.34695178E-06-8.75489269E-07 0.00000000E+00 0.00000000E+00

128 9.99859652E-01-1.06728890E-05 5.03294613E-04-9.32887033E-04-9.91855359E-04

$1290.00000000 \mathrm{E}+002.06580782 \mathrm{E}-04$ 8.00934119E-04 0.00000000E+00 6.49191049E-04

$1305.36013851 \mathrm{E}-04$ 6.34510303E-04 1.78003997E-05 0.00000000E+00 0.00000000E+00

1 31-1.30038039E-07-3.06819804E-07 1.89690240E-06-5.54869106E-07 1.68863928E-07

$1320.00000000 \mathrm{E}+00$ 9.19562666E-06 7.91749823E-06 0.00000000E+00 7.86749811E-07

133 5.35126593E-08-1.48716184E-06 9.63051515E-07 0.00000000E+00 0.00000000E+00

$134-1.37013755 \mathrm{E}-06-6.70959536 \mathrm{E}-06$ 4.71066403E-05 7.73542789E-07-5.00043783E-07

$1350.00000000 \mathrm{E}+003.87668043 \mathrm{E}-07-7.19033703 \mathrm{E}-060.00000000 \mathrm{E}+00-5.12818385 \mathrm{E}-06$

1 36-4.20107465E-06-1.93045883E-06 3.96264343E-07 0.00000000E+00 0.00000000E+00 137 1.54457819E-04 2.51958285E-04 3.93812826E-06-2.68947594E-05 2.09907315E-06

1 38-2.90838431E-04-3.14338623E-06 9.64807604E-05 1.49828140E-06-1.06567583E-05

$139-5.51633550 \mathrm{E}-08$ 1.53776044E-04 1.65678355E-05 1.06135174E-05-6.03904980E-06

1 40-1.99012199E-05

\$END 


\title{
B.3 Energy calculations for ground state
}

\author{
$\$$ CONTRL SCFTYP=MCSCF RUNTYP=ENERGY MAXIT=100 COORD=CART \$END \\ $\$ M C S C F$ CISTEP $=$ GUGA MAXIT $=200$ \$END \\ $\$ S Y S T E M$ MEMORY $=100000000$ TIMLIM $=100000$ \$END \\ $\$$ BASIS GBASIS $=$ DH NDFUNC $=1$ POLAR $=$ DUNNING \$END \\ \$GUESS GUESS $=$ MOREAD NORB $=196$ \$END \\ $\$$ DRT GROUP $=\mathrm{C} 1 \mathrm{FORS}=. \mathrm{T} . \mathrm{NMCC}=38 \mathrm{NDOC}=5 \mathrm{NVAL}=3 \$ \$ \mathrm{END}$ \\ \$GUGDIA NSTATE $=6$ ITERMX $=200$ \$END \\ $\$$ GUGDM NFLGDM(1) $=1,0,0,0,0$ IROOT $=1$ \$END \\ \$GUGDM2 WSTATE(1) $=1.0,1.0,1.0,1.0$ \$END \\ $\$ S T A T P T$ NSTEP $=100$ DXMAX $=0.05$ \$END \\ \$DATA
}

CNH 1

$\begin{array}{lrrrr}\mathrm{C} & 6.0 & 0.4163908690 & 0.2326604093 & 0.0000000000 \\ \mathrm{C} & 6.0 & 0.9667952878 & -1.0537230967 & 0.0000000000 \\ \mathrm{C} & 6.0 & 2.3507625055 & -1.2453757682 & 0.0000000000 \\ \mathrm{C} & 6.0 & 3.2063828898 & -0.1580262437 & 0.0000000000 \\ \mathrm{C} & 6.0 & 2.6832774224 & 1.1383276474 & 0.0000000000 \\ \mathrm{C} & 6.0 & 1.3064695523 & 1.3239362194 & 0.0000000000 \\ \mathrm{C} & 6.0 & -1.0349985450 & 0.5002690085 & 0.0000000000 \\ \mathrm{C} & 6.0 & -2.0316980051 & -0.4086321816 & 0.0000000000 \\ \mathrm{C} & 6.0 & -3.4461096556 & 0.0395213473 & 0.0000000000 \\ \mathrm{O} & 8.0 & 4.5530882951 & -0.2829549599 & 0.0000000000 \\ \mathrm{O} & 8.0 & -4.2906324743 & -0.9914256329 & 0.0000000000 \\ \mathrm{O} & 8.0 & -3.8252672732 & 1.1707562683 & 0.0000000000 \\ \mathrm{H} & 1.0 & 4.8068995248 & -1.1948499186 & 0.0000000000 \\ \mathrm{H} & 1.0 & 2.7494932557 & -2.2465680163 & 0.0000000000 \\ \mathrm{H} & 1.0 & 0.3327298818 & -1.9208178077 & 0.0000000000 \\ \mathrm{H} & 1.0 & -1.8690888247 & -1.4693394788 & 0.0000000000 \\ \mathrm{H} & 1.0 & -5.1766588746 & -0.6444362772 & 0.0000000000 \\ \mathrm{H} & 1.0 & -1.3238705391 & 1.5366726209 & 0.0000000000 \\ \mathrm{H} & 1.0 & 0.9158711190 & 2.3263321660 & 0.0000000000 \\ \mathrm{H} & 1.0 & 3.3591143628 & 1.9735545752 & 0.0000000000\end{array}$

\$END 


\section{\$VEC}

1 1-5.74241546E-06-2.66200965E-05 4.43162619E-04 6.53775727E-06-1.27549490E-05

12 0.00000000E+00 6.36777468E-05 8.43559247E-04 0.00000000E+00-1.44936503E-06

1 3-1.44709527E-05-8.84764643E-06 2.59455122E-06 0.00000000E+00 0.00000000E+00

1 4-2.70395199E-06-8.44013234E-06 4.35050407E-04 1.63089414E-05 5.07357965E-06

$150.00000000 \mathrm{E}+00-4.91949359 \mathrm{E}-04-6.90824388 \mathrm{E}-050.00000000 \mathrm{E}+00-1.56509379 \mathrm{E}-05$

16 9.71581000E-07-7.17051313E-08-3.42337309E-06 0.00000000E+00 0.00000000E +00

17 8.99142908E-06 4.61815722E-07-6.64216422E-04-1.65539169E-05-1.58923874E-05

$180.00000000 \mathrm{E}+00-2.13328362 \mathrm{E}-04-1.26913909 \mathrm{E}-040.00000000 \mathrm{E}+004.11488616 \mathrm{E}-05$

$193.89178657 \mathrm{E}-05-8.84622402 \mathrm{E}-07$ 1.05841921E-05 0.00000000E $+000.00000000 \mathrm{E}+00$

1 10-3.03409829E-05 2.13240688E-04-1.68660084E-04 1.16820033E-05 4.25030490E-07

$1110.00000000 \mathrm{E}+00-7.54699763 \mathrm{E}-04-1.13369523 \mathrm{E}-03$ 0.00000000E+00-2.34113398E-04

1 12-8.59849466E-05-2.88608913E-05 4.86457109E-05 0.00000000E+00 0.00000000E+00

1 13-7.57993948E-06-5.03436691E-05 5.89171462E-04 3.77727093E-06-2.57647341E-05

$1140.00000000 \mathrm{E}+003.96460058 \mathrm{E}-04-3.69489200 \mathrm{E}-040.00000000 \mathrm{E}+00-1.28726388 \mathrm{E}-05$

115 9.94013808E-06-1.91893094E-05 2.55661387E-06 0.00000000E+00 0.00000000E+00

116 5.34356427E-06 3.58481752E-05-4.39086330E-04-4.07201758E-06 1.61612011E-05

$1170.00000000 \mathrm{E}+00$ 4.25860925E-04 2.46714595E-04 0.00000000E+00 1.42952089E-06

118 4.16124916E-07 1.49514264E-05 4.43499709E-06 0.00000000E+00 0.00000000E+00

$1193.06023017 \mathrm{E}-06$ 1.70904338E-05-2.84938832E-04-9.09301732E-06-5.79409850E-06

$1200.00000000 \mathrm{E}+00-1.01885552 \mathrm{E}-04-2.97158342 \mathrm{E}-040.00000000 \mathrm{E}+00-2.40642669 \mathrm{E}-06$

121 3.31660701E-06 7.36769003E-06-5.91733780E-06 0.00000000E+00 0.00000000E+00

122 9.72089168E-07 8.19045045E-07-7.42411463E-05 1.70203048E-06-2.21183944E-06

123 0.00000000E+00-8.52962573E-05 1.42079724E-04 0.00000000E+00 6.47132287E-06

124 3.04819364E-06 7.13751466E-07 2.37669079E-06 0.00000000E+00 0.00000000E+00

125 2.91189040E-06 1.24524140E-05-1.59508949E-04-7.94965594E-07-2.23814707E-08

$1260.00000000 \mathrm{E}+00-5.33905628 \mathrm{E}-05-4.75221528 \mathrm{E}-05$ 0.00000000E+00 2.18769934E-06

127 4.47743449E-06 7.34695178E-06-8.75489269E-07 0.00000000E+00 0.00000000E+00

128 9.99859652E-01-1.06728890E-05 5.03294613E-04-9.32887033E-04-9.91855359E-04

$1290.00000000 \mathrm{E}+002.06580782 \mathrm{E}-04$ 8.00934119E-04 0.00000000E+00 6.49191049E-04

$1305.36013851 \mathrm{E}-04$ 6.34510303E-04 1.78003997E-05 0.00000000E+00 0.00000000E+00

1 31-1.30038039E-07-3.06819804E-07 1.89690240E-06-5.54869106E-07 1.68863928E-07

$1320.00000000 \mathrm{E}+00$ 9.19562666E-06 7.91749823E-06 0.00000000E+00 7.86749811E-07

133 5.35126593E-08-1.48716184E-06 9.63051515E-07 0.00000000E+00 0.00000000E+00

$134-1.37013755 \mathrm{E}-06-6.70959536 \mathrm{E}-06$ 4.71066403E-05 7.73542789E-07-5.00043783E-07

$1350.00000000 \mathrm{E}+003.87668043 \mathrm{E}-07-7.19033703 \mathrm{E}-060.00000000 \mathrm{E}+00-5.12818385 \mathrm{E}-06$

1 36-4.20107465E-06-1.93045883E-06 3.96264343E-07 0.00000000E+00 0.00000000E+00

137 1.54457819E-04 2.51958285E-04 3.93812826E-06-2.68947594E-05 2.09907315E-06

1 38-2.90838431E-04-3.14338623E-06 9.64807604E-05 1.49828140E-06-1.06567583E-05

$139-5.51633550 \mathrm{E}-08$ 1.53776044E-04 1.65678355E-05 1.06135174E-05-6.03904980E-06

1 40-1.99012199E-05

\$END 


\section{C}

\section{APPENDIX: Energy and structure}

\section{parameters calculations of excited state for}

\section{p-coumaric acid molecule}

In this chapter the energy and the structure calculations of the excited state are given using the GAMESS US software, the Multi Configuration Self Consistent Field method. As before we are given here the program structure for optimizing the system, for calculating the normal modes (via calculating the Hessian matrix) and also the calculations of the energies for excited states. 


\title{
C.1 Optimize the second excited state for p-coumaric acid
}

\author{
\$CONTRL SCFTYP=MCSCF RUNTYP=OPTIMIZE EXETYP=RUN \\ MAXIT $=100 \mathrm{COORD}=\mathrm{UNIQUE}$ \\ \$END \\ $\$$ MCSCF CISTEP $=$ GUGA MAXIT $=200$ \$END \\ $\$$ SYSTEM MEMORY $=300000000$ TIMLIM $=6000000.0 \$ E N D$ \\ $\$$ BASIS GBASIS $=$ DH NDFUNC $=1$ POLAR $=$ DUNNING $\$ E N D$ \\ \$GUESS GUESS $=$ MOREAD NORB $=196 \$$ END \\ $\$$ DRT GROUP $=\mathrm{C} 1$ FORS $=$.T. $\mathrm{NMCC}=38 \mathrm{NDOC}=5 \mathrm{NVAL}=3$ \$END \\ \$GUGDIA NSTATE $=6$ ITERMX $=200$ \$END \\ $\$$ GUGDM NFLGDM(1) $=0,1,0,0,0$ IROOT $=2$ \$END \\ \$GUGDM2 WSTATE $(1)=0.0,1.0,0.0$ \$END \\ $\$$ STATPT NSTEP $=100$ DXMAX $=0.05$ \$END \\ \$DATA
}

\section{$\mathrm{CNH} 1$}

$\begin{array}{lrrrr}\mathrm{C} & 6.0 & 0.3857684051 & 0.3208999453 & 0.0000000000 \\ \mathrm{C} & 6.0 & 0.9189011047 & -1.0705721303 & 0.0000000000 \\ \mathrm{C} & 6.0 & 2.2821791178 & -1.2703519400 & 0.0000000000 \\ \mathrm{C} & 6.0 & 3.1581421065 & -0.1627619802 & 0.0000000000 \\ \mathrm{C} & 6.0 & 2.6763870787 & 1.2026049709 & 0.0000000000 \\ \mathrm{C} & 6.0 & 1.3489316597 & 1.4174548998 & 0.0000000000 \\ \mathrm{C} & 6.0 & -0.9495508560 & 0.5487417286 & 0.0000000000 \\ \mathrm{C} & 6.0 & -2.0188598910 & -0.4944398517 & 0.0000000000 \\ \mathrm{C} & 6.0 & -3.3840650816 & -0.0585298875 & 0.0000000000 \\ \mathrm{O} & 8.0 & 4.4846074717 & -0.3008038006 & 0.0000000000 \\ \mathrm{O} & 8.0 & -4.2718031733 & -1.0677972721 & 0.0000000000 \\ \mathrm{O} & 8.0 & -3.7614996214 & 1.0924560514 & 0.0000000000 \\ \mathrm{H} & 1.0 & 4.7426533069 & -1.2131688007 & 0.0000000000 \\ \mathrm{H} & 1.0 & 2.6924726469 & -2.2661664687 & 0.0000000000 \\ \mathrm{H} & 1.0 & 0.2491573923 & -1.9038093823 & 0.0000000000 \\ \mathrm{H} & 1.0 & -1.8048499967 & -1.5412924829 & 0.0000000000 \\ \mathrm{H} & 1.0 & -5.1363756758 & -0.6739316627 & 0.0000000000 \\ \mathrm{H} & 1.0 & -1.3114458701 & 1.5615393684 & 0.0000000000 \\ \mathrm{H} & 1.0 & 0.9664943095 & 2.4227448743 & 0.0000000000 \\ \mathrm{H} & 1.0 & 3.3965770326 & 1.9981162128 & 0.0000000000 \\ \text { SEND } & & & & \end{array}$




\section{\$VEC}

1 1-5.34936986E-06-2.59054439E-05 4.37525072E-04 7.34983648E-06-1.29202763E-05

$120.00000000 \mathrm{E}+005.77913946 \mathrm{E}-05$ 8.40388037E-04 0.00000000E+00-1.09911163E-06

1 3-1.40838966E-05-8.83000701E-06 2.58739131E-06 0.00000000E+00 0.00000000E+00

1 4-2.55280796E-06-7.83602766E-06 4.33781096E-04 1.73162492E-05 6.19662657E-06

$150.00000000 \mathrm{E}+00-4.87566158 \mathrm{E}-04-6.82310236 \mathrm{E}-050.00000000 \mathrm{E}+00-1.55967639 \mathrm{E}-05$

$167.38705060 \mathrm{E}-07-2.56353495 \mathrm{E}-07-3.45157617 \mathrm{E}-060.00000000 \mathrm{E}+000.00000000 \mathrm{E}+00$

$179.00970631 \mathrm{E}-06$ 1.98388096E-07-6.62008741E-04-1.76509596E-05-1.41118283E-05

$180.00000000 \mathrm{E}+00-2.15406823 \mathrm{E}-04-1.26933319 \mathrm{E}-040.00000000 \mathrm{E}+004.12682983 \mathrm{E}-05$

$193.97382599 \mathrm{E}-05-7.64269861 \mathrm{E}-07$ 1.07809154E-05 0.00000000E $+000.00000000 \mathrm{E}+00$

1 10-3.12761130E-05 2.11869318E-04-1.47929846E-04 8.78987051E-06 1.40086759E-06

$1110.00000000 \mathrm{E}+00-7.34033733 \mathrm{E}-04-1.13142354 \mathrm{E}-03$ 0.00000000E+00-2.40558050E-04

1 12-8.84184551E-05-2.61067893E-05 4.88551336E-05 0.00000000E+00 0.00000000E+00

1 13-7.34669106E-06-5.04971100E-05 5.88585224E-04 1.92685167E-06-2.56728121E-05

$1140.00000000 \mathrm{E}+00$ 3.89193093E-04-3.62742971E-04 0.00000000E+00-1.29561814E-05

115 1.04869775E-05-1.88950873E-05 2.56027883E-06 0.00000000E+00 0.00000000E+00

116 5.68371998E-06 3.69798451E-05-4.41778005E-04-2.61928189E-06 1.50285770E-05

$1170.00000000 \mathrm{E}+00$ 4.20986372E-04 2.43212939E-04 0.00000000E+00 1.67007335E-06

$1184.33539282 \mathrm{E}-07$ 1.48415578E-05 4.38441592E-06 0.00000000E+00 0.00000000E+00

119 2.23269994E-06 1.85121526E-05-2.82577107E-04-1.05022784E-05-4.95948800E-06

$1200.00000000 \mathrm{E}+00-1.02149472 \mathrm{E}-04-2.96290910 \mathrm{E}-040.00000000 \mathrm{E}+00-2.39751213 \mathrm{E}-06$

121 3.49238496E-06 7.37382908E-06-5.89438236E-06 0.00000000E+00 0.00000000E+00

122 6.83019640E-07 1.28143502E-06-7.48293777E-05 2.18127137E-06-1.28018678E-06

123 0.00000000E+00-8.46359856E-05 1.38986091E-04 0.00000000E+00 6.39471133E-06

124 3.09825272E-06 7.89486143E-07 2.46482141E-06 0.00000000E+00 0.00000000E+00

$1252.87695520 \mathrm{E}-06$ 1.22776583E-05-1.57286530E-04-7.68444876E-07-5.57364110E-08

$1260.00000000 \mathrm{E}+00-5.25722138 \mathrm{E}-05-4.71780502 \mathrm{E}-050.00000000 \mathrm{E}+00$ 2.20522232E-06

127 4.43070034E-06 7.28634730E-06-8.58618957E-07 0.00000000E+00 0.00000000E+00

128 9.99866682E-01-2.92060915E-05 4.75162407E-04-9.33886148E-04-9.93480447E-04

$1290.00000000 \mathrm{E}+00$ 2.11390021E-04 8.02971104E-04 0.00000000E+00 6.72202665E-04

130 5.61369037E-04 6.31513146E-04 1.72102941E-05 0.00000000E+00 0.00000000E+00

1 31-1.10460377E-07-3.36220747E-07 1.69699456E-06-3.59052885E-07 1.30187815E-07

$1320.00000000 \mathrm{E}+00$ 9.07012462E-06 7.86449031E-06 0.00000000E+00 7.81914434E-07

133 8.23228987E-08-1.45421053E-06 9.64343888E-07 0.00000000E+00 0.00000000E+00

$134-1.32013342 \mathrm{E}-06-6.56445456 \mathrm{E}-06$ 4.63383251E-05 6.90447237E-07-4.16541862E-07

$1350.00000000 \mathrm{E}+002.89723296 \mathrm{E}-07-7.07085997 \mathrm{E}-06$ 0.00000000E+00-5.02897308E-06

1 36-4.11081140E-06-1.88211780E-06 3.96443368E-07 0.00000000E+00 0.00000000E+00

137 1.53152135E-04 2.50165394E-04 3.17519577E-06-2.62292204E-05 1.62148210E-06

1 38-2.88489765E-04-3.28615401E-06 9.45224933E-05 1.37399451E-06-1.06215671E-05

139 1.08234631E-06 1.53273696E-04 1.61468488E-05 1.20696170E-05-6.24087895E-06

1 40-1.94741424E-05

\$END 


\section{C.2 Hessian calculations for the excited state}

$\$$ CONTRL SCFTYP=MCSCF RUNTYP=Hessian EXETYP=RUN MAXIT=100 COORD $=$ UNIQUE $\$ E N D$

$\$$ MCSCF CISTEP $=$ GUGA MAXIT $=200$ \$END

$\$$ SYSTEM MEMORY $=300000000$ TIMLIM $=6000000.0$ \$END

$\$$ BASIS GBASIS $=$ DH NDFUNC $=1$ POLAR $=$ DUNNING $\$ E N D$

\$GUESS GUESS $=$ MOREAD NORB $=196$ \$END

$\$$ DRT GROUP $=\mathrm{C} 1 \mathrm{FORS}=$.T. $\mathrm{NMCC}=38 \mathrm{NDOC}=5 \mathrm{NVAL}=3$ \$END

\$GUGDIA NSTATE $=6$ ITERMX $=200$ \$END

$\$$ GUGDM NFLGDM(1) $=0,1,0,0,0$ IROOT $=2$ \$END

\$GUGDM2 WSTATE(1) $=0.0,1.0,0.0,0.0$ \$END

$\$$ STATPT NSTEP $=100$ DXMAX $=0.05$ \$END

\$DATA

CNH 1

$\begin{array}{lrrrr}\mathrm{C} & 6.0 & 0.3858392876 & 0.3207662431 & 0.0000000000 \\ \mathrm{C} & 6.0 & 0.9190347083 & -1.0703945249 & 0.0000000000 \\ \mathrm{C} & 6.0 & 2.2821271014 & -1.2705053923 & 0.0000000000 \\ \mathrm{C} & 6.0 & 3.1584041349 & -0.1624754731 & 0.0000000000 \\ \mathrm{C} & 6.0 & 2.6764145185 & 1.2027207773 & 0.0000000000 \\ \mathrm{C} & 6.0 & 1.3490315918 & 1.4176541378 & 0.0000000000 \\ \mathrm{C} & 6.0 & -0.9497848353 & 0.5486055532 & 0.0000000000 \\ \mathrm{C} & 6.0 & -2.0191437021 & -0.4946943167 & 0.0000000000 \\ \mathrm{C} & 6.0 & -3.3840241468 & -0.0585963029 & 0.0000000000 \\ \mathrm{O} & 8.0 & 4.4848422009 & -0.3008686034 & 0.0000000000 \\ \mathrm{O} & 8.0 & -4.2719217739 & -1.0677967070 & 0.0000000000 \\ \mathrm{O} & 8.0 & -3.7614638260 & 1.0924772990 & 0.0000000000 \\ \mathrm{H} & 1.0 & 4.7424633123 & -1.2133143861 & 0.0000000000 \\ \mathrm{H} & 1.0 & 2.6924794414 & -2.2663171731 & 0.0000000000 \\ \mathrm{H} & 1.0 & 0.2492619566 & -1.9035353228 & 0.0000000000 \\ \mathrm{H} & 1.0 & -1.8048652018 & -1.5414838214 & 0.0000000000 \\ \mathrm{H} & 1.0 & -5.1363299695 & -0.6736503905 & 0.0000000000 \\ \mathrm{H} & 1.0 & -1.3116987013 & 1.5613439460 & 0.0000000000 \\ \mathrm{H} & 1.0 & 0.9665201824 & 2.4228907632 & 0.0000000000 \\ \mathrm{H} & 1.0 & 3.3966351872 & 1.9981060865 & 0.0000000000\end{array}$

\$END 


\section{\$VEC}

1 1-5.34926806E-06-2.59050569E-05 4.37520720E-04 7.34983441E-06-1.29215837E-05

$120.00000000 \mathrm{E}+00$ 5.78051378E-05 8.40429991E-04 0.00000000E+00-1.09868910E-06

1 3-1.40840233E-05-8.82969143E-06 2.58769872E-06 0.00000000E+00 0.00000000E+00

1 4-2.55229762E-06-7.83453347E-06 4.33765070E-04 1.73165701E-05 6.19634412E-06

$150.00000000 \mathrm{E}+00-4.87598477 \mathrm{E}-04-6.82041254 \mathrm{E}-050.00000000 \mathrm{E}+00-1.55948948 \mathrm{E}-05$

16 7.39718129E-07-2.56080217E-07-3.45248670E-06 0.00000000E+00 0.00000000E+00

$179.00936465 \mathrm{E}-06$ 1.91943866E-07-6.62020546E-04-1.76551457E-05-1.41138962E-05

$180.00000000 \mathrm{E}+00-2.15452215 \mathrm{E}-04-1.26956885 \mathrm{E}-040.00000000 \mathrm{E}+004.12629755 \mathrm{E}-05$

$193.97359284 \mathrm{E}-05-7.62109466 \mathrm{E}-07$ 1.07804780E-05 0.00000000E $+000.00000000 \mathrm{E}+00$

1 10-3.12757315E-05 2.11864302E-04-1.47904112E-04 8.78414310E-06 1.40415620E-06

$1110.00000000 \mathrm{E}+00-7.34039274 \mathrm{E}-04-1.13152128 \mathrm{E}-03$ 0.00000000E+00-2.40566743E-04

1 12-8.84133145E-05-2.61129711E-05 4.88548552E-05 0.00000000E+00 0.00000000E+00

1 13-7.34739785E-06-5.04977524E-05 5.88617420E-04 1.92749869E-06-2.56737239E-05

$1140.00000000 \mathrm{E}+00$ 3.89229684E-04-3.62771455E-04 0.00000000E+00-1.29550460E-05

115 1.04855558E-05-1.88976035E-05 2.56013247E-06 0.00000000E+00 0.00000000E+00

$1165.68341582 \mathrm{E}-06$ 3.69787989E-05-4.41773066E-04-2.62000224E-06 1.50284841E-05

$1170.00000000 \mathrm{E}+004.21020222 \mathrm{E}-042.43225682 \mathrm{E}-040.00000000 \mathrm{E}+001.66885746 \mathrm{E}-06$

$1184.32733416 \mathrm{E}-07$ 1.48415287E-05 4.38444665E-06 0.00000000E+00 0.00000000E+00

119 2.23267579E-06 1.85115621E-05-2.82572312E-04-1.05022541E-05-4.96020777E-06

$1200.00000000 \mathrm{E}+00-1.02146554 \mathrm{E}-04-2.96301663 \mathrm{E}-040.00000000 \mathrm{E}+00-2.39784241 \mathrm{E}-06$

121 3.49245789E-06 7.37367757E-06-5.89450350E-06 0.00000000E+00 0.00000000E+00

122 6.83005695E-07 1.28069677E-06-7.48271866E-05 2.18097084E-06-1.28037285E-06

123 0.00000000E+00-8.46406448E-05 1.38986405E-04 0.00000000E+00 6.39450720E-06

124 3.09805711E-06 7.89564831E-07 2.46482448E-06 0.00000000E+00 0.00000000E+00

125 2.87699899E-06 1.22781403E-05-1.57291916E-04-7.68569167E-07-5.57153336E-08

$1260.00000000 \mathrm{E}+00-5.25733973 \mathrm{E}-05-4.71769735 \mathrm{E}-050.00000000 \mathrm{E}+00$ 2.20528523E-06

127 4.43079110E-06 7.28641330E-06-8.58754675E-07 0.00000000E+00 0.00000000E+00

128 9.99866689E-01-2.92229945E-05 4.75133900E-04-9.33898136E-04-9.93492460E-04

$1290.00000000 \mathrm{E}+002.11406930 \mathrm{E}-04$ 8.02995665E-04 0.00000000E+00 6.72217680E-04

130 5.61379053E-04 6.31544237E-04 1.72100352E-05 0.00000000E+00 0.00000000E+00

1 31-1.10478683E-07-3.36486743E-07 1.69807759E-06-3.59064841E-07 1.30237061E-07

$1320.00000000 \mathrm{E}+00$ 9.07012083E-06 7.86470805E-06 0.00000000E+00 7.81704526E-07

133 8.24052015E-08-1.45440577E-06 9.64391399E-07 0.00000000E+00 0.00000000E+00

$134-1.32015369 \mathrm{E}-06-6.56457446 \mathrm{E}-06$ 4.63389670E-05 6.90503019E-07-4.16535685E-07

$1350.00000000 \mathrm{E}+002.90122841 \mathrm{E}-07-7.07104323 \mathrm{E}-060.00000000 \mathrm{E}+00-5.02895210 \mathrm{E}-06$

1 36-4.11096131E-06-1.88217473E-06 3.96462188E-07 0.00000000E+00 0.00000000E+00

137 1.53145498E-04 2.50165990E-04 3.17364106E-06-2.62417561E-05 1.62186424E-06

1 38-2.88487632E-04-3.28591365E-06 9.45204752E-05 1.37389864E-06-1.06216889E-05

139 1.08162653E-06 1.53280279E-04 1.61468115E-05 1.20702455E-05-6.24178986E-06

1 40-1.94739804E-05

\$END 


\section{C.3 Energy calculation for excited state}

$\$$ CONTRL SCFTYP=MCSCF RUNTYP=ENERGY EXETYP=RUN MAXIT $=100 \mathrm{COORD}=\mathrm{UNIQUE}$

\$END

$\$$ MCSCF CISTEP $=$ GUGA MAXIT $=200$ \$END

$\$$ SYSTEM MEMORY $=300000000$ TIMLIM $=6000000.0$ \$END

$\$$ BASIS GBASIS $=$ DH NDFUNC $=1$ POLAR $=$ DUNNING $\$ E N D$

$\$$ GUESS GUESS $=$ MOREAD NORB $=196$ \$END

$\$ D R T$ GROUP $=\mathrm{C} 1 \mathrm{FORS}=. \mathrm{T} . \mathrm{NMCC}=38 \mathrm{NDOC}=5 \mathrm{NVAL}=3 \$ \mathrm{END}$

\$GUGDIA NSTATE $=6$ ITERMX $=200$ \$END

$\$$ GUGDM NFLGDM(1) $=0,1,0,0,0$ IROOT $=2$ \$END

\$GUGDM2 WSTATE(1)=1.0,1.0,1.0,1.0 \$END

$\$$ STATPT NSTEP $=100$ DXMAX $=0.05$ \$END

\$DATA

\section{CNH 1}

$\begin{array}{lrrrr}\text { C } & 6.0 & 0.3858392876 & 0.3207662431 & 0.0000000000 \\ \text { C } & 6.0 & 0.9190347083 & -1.0703945249 & 0.0000000000 \\ \text { C } & 6.0 & 2.2821271014 & -1.2705053923 & 0.0000000000 \\ \text { C } & 6.0 & 3.1584041349 & -0.1624754731 & 0.0000000000 \\ \text { C } & 6.0 & 2.6764145185 & 1.2027207773 & 0.0000000000 \\ \text { C } & 6.0 & 1.3490315918 & 1.4176541378 & 0.0000000000 \\ \text { C } & 6.0 & -0.9497848353 & 0.5486055532 & 0.0000000000 \\ \text { C } & 6.0 & -2.0191437021 & -0.4946943167 & 0.0000000000 \\ \text { C } & 6.0 & -3.3840241468 & -0.0585963029 & 0.0000000000 \\ \text { O } & 8.0 & 4.4848422009 & -0.3008686034 & 0.0000000000 \\ \text { O } & 8.0 & -4.2719217739 & -1.0677967070 & 0.0000000000 \\ \text { O } & 8.0 & -3.7614638260 & 1.0924772990 & 0.0000000000 \\ \text { H } & 1.0 & 4.7424633123 & -1.2133143861 & 0.0000000000 \\ \text { H } & 1.0 & 2.6924794414 & -2.2663171731 & 0.0000000000 \\ \text { H } & 1.0 & 0.2492619566 & -1.9035353228 & 0.0000000000 \\ \text { H } & 1.0 & -1.8048652018 & -1.5414838214 & 0.0000000000 \\ \text { H } & 1.0 & -5.1363299695 & -0.6736503905 & 0.0000000000 \\ \text { H } & 1.0 & -1.3116987013 & 1.5613439460 & 0.0000000000 \\ \text { H } & 1.0 & 0.9665201824 & 2.4228907632 & 0.0000000000 \\ \text { H } & 1.0 & 3.3966351872 & 1.9981060865 & 0.0000000000 \\ \text { SEND } & & & & \end{array}$




\section{\$VEC}

1 1-5.34926806E-06-2.59050569E-05 4.37520720E-04 7.34983441E-06-1.29215837E-05

$120.00000000 \mathrm{E}+00$ 5.78051378E-05 8.40429991E-04 0.00000000E+00-1.09868910E-06

1 3-1.40840233E-05-8.82969143E-06 2.58769872E-06 0.00000000E+00 0.00000000E+00

1 4-2.55229762E-06-7.83453347E-06 4.33765070E-04 1.73165701E-05 6.19634412E-06

$150.00000000 \mathrm{E}+00-4.87598477 \mathrm{E}-04-6.82041254 \mathrm{E}-050.00000000 \mathrm{E}+00-1.55948948 \mathrm{E}-05$

16 7.39718129E-07-2.56080217E-07-3.45248670E-06 0.00000000E+00 0.00000000E+00

$179.00936465 \mathrm{E}-06$ 1.91943866E-07-6.62020546E-04-1.76551457E-05-1.41138962E-05

$180.00000000 \mathrm{E}+00-2.15452215 \mathrm{E}-04-1.26956885 \mathrm{E}-040.00000000 \mathrm{E}+004.12629755 \mathrm{E}-05$

$193.97359284 \mathrm{E}-05-7.62109466 \mathrm{E}-07$ 1.07804780E-05 0.00000000E $+000.00000000 \mathrm{E}+00$

1 10-3.12757315E-05 2.11864302E-04-1.47904112E-04 8.78414310E-06 1.40415620E-06

$1110.00000000 \mathrm{E}+00-7.34039274 \mathrm{E}-04-1.13152128 \mathrm{E}-03$ 0.00000000E+00-2.40566743E-04

1 12-8.84133145E-05-2.61129711E-05 4.88548552E-05 0.00000000E+00 0.00000000E+00

1 13-7.34739785E-06-5.04977524E-05 5.88617420E-04 1.92749869E-06-2.56737239E-05

$1140.00000000 \mathrm{E}+00$ 3.89229684E-04-3.62771455E-04 0.00000000E+00-1.29550460E-05

115 1.04855558E-05-1.88976035E-05 2.56013247E-06 0.00000000E+00 0.00000000E+00

$1165.68341582 \mathrm{E}-06$ 3.69787989E-05-4.41773066E-04-2.62000224E-06 1.50284841E-05

$1170.00000000 \mathrm{E}+004.21020222 \mathrm{E}-042.43225682 \mathrm{E}-040.00000000 \mathrm{E}+001.66885746 \mathrm{E}-06$

$1184.32733416 \mathrm{E}-07$ 1.48415287E-05 4.38444665E-06 0.00000000E+00 0.00000000E+00

119 2.23267579E-06 1.85115621E-05-2.82572312E-04-1.05022541E-05-4.96020777E-06

$1200.00000000 \mathrm{E}+00-1.02146554 \mathrm{E}-04-2.96301663 \mathrm{E}-040.00000000 \mathrm{E}+00-2.39784241 \mathrm{E}-06$

121 3.49245789E-06 7.37367757E-06-5.89450350E-06 0.00000000E+00 0.00000000E+00

122 6.83005695E-07 1.28069677E-06-7.48271866E-05 2.18097084E-06-1.28037285E-06

123 0.00000000E+00-8.46406448E-05 1.38986405E-04 0.00000000E+00 6.39450720E-06

124 3.09805711E-06 7.89564831E-07 2.46482448E-06 0.00000000E+00 0.00000000E+00

125 2.87699899E-06 1.22781403E-05-1.57291916E-04-7.68569167E-07-5.57153336E-08

$1260.00000000 \mathrm{E}+00-5.25733973 \mathrm{E}-05-4.71769735 \mathrm{E}-050.00000000 \mathrm{E}+00$ 2.20528523E-06

127 4.43079110E-06 7.28641330E-06-8.58754675E-07 0.00000000E+00 0.00000000E+00

128 9.99866689E-01-2.92229945E-05 4.75133900E-04-9.33898136E-04-9.93492460E-04

$1290.00000000 \mathrm{E}+002.11406930 \mathrm{E}-04$ 8.02995665E-04 0.00000000E+00 6.72217680E-04

130 5.61379053E-04 6.31544237E-04 1.72100352E-05 0.00000000E+00 0.00000000E+00

1 31-1.10478683E-07-3.36486743E-07 1.69807759E-06-3.59064841E-07 1.30237061E-07

$1320.00000000 \mathrm{E}+00$ 9.07012083E-06 7.86470805E-06 0.00000000E+00 7.81704526E-07

133 8.24052015E-08-1.45440577E-06 9.64391399E-07 0.00000000E+00 0.00000000E+00

$134-1.32015369 \mathrm{E}-06-6.56457446 \mathrm{E}-06$ 4.63389670E-05 6.90503019E-07-4.16535685E-07

$1350.00000000 \mathrm{E}+002.90122841 \mathrm{E}-07-7.07104323 \mathrm{E}-060.00000000 \mathrm{E}+00-5.02895210 \mathrm{E}-06$

1 36-4.11096131E-06-1.88217473E-06 3.96462188E-07 0.00000000E+00 0.00000000E+00

137 1.53145498E-04 2.50165990E-04 3.17364106E-06-2.62417561E-05 1.62186424E-06

1 38-2.88487632E-04-3.28591365E-06 9.45204752E-05 1.37389864E-06-1.06216889E-05

139 1.08162653E-06 1.53280279E-04 1.61468115E-05 1.20702455E-05-6.24178986E-06

1 40-1.94739804E-05

\$END 


\section{D}

\section{APPENDIX: Transition dipole moment}

\section{calculations}

In this chapter are explained the way in which we have calculated the transition dipole moment for benzene and stilbene molecules.

First, stilbene has a large non-zero TDM in its equilibrium geometry, thermal distortions from the equilibrium structure induce additional TDM, but this effect was neglected, therefore TDM in stilbene was treated in Condo approximation. So it means that we have constant coupling term between the PES, and its amplitude plays no role in linear response regime (spectrum is proportional to the square of transition dipole moment, but we do not calculate its amplitude, just spectral shape).

Second, benzene has no transition dipole moment in its equilibrium geometry due to high symmetry, therefore it only has induced TDM. To calculate its components the following steps were done. For some small (but fixed) displacement along every normal mode of the ground and the first excited state the corresponding cartesian coordinates of such a distorted configuration were calculated (normal mode vectors are known, it was written a small FORTRAN program to do it). Then the transition dipole moment for every distorted configuration was calculated. 
In GAMESS this is a two-step calculation. First, for every electron state of interest the ground and the first excited one) we need single-point energy calculation. Second, the orbitals and the gradients have to be provided for the transition dipole moment calculation.

\section{D.1 Equilibrium ground state for benzene molecule}

$\$ C O N T R L$ SCFTYP=MCSCF RUNTYP=ENERGY EXETYP=RUN MAXIT $=100$ COORD=UNIQUE \$END

\$MCSCF CISTEP $=$ GUGA MAXIT $=100$ \$END

\$SYSTEM MEMORY=40000000 TIMLIM=6000000.0 \$END

$\$$ BASIS GBASIS $=\mathrm{N} 31$ NGAUSS $=6 \mathrm{NDFUNC}=1$ \$END

\$GUESS GUESS $=$ MOREAD NORB $=102$ \$END

$\$ D R T$ GROUP $=$ C1 FORS $=$. T. $\mathrm{NMCC}=18 \mathrm{NDOC}=3 \mathrm{NVAL}=3$ \$END

\$GUGDIA NSTATE $=4$ ITERMX $=200$ \$END

$\$$ LAGRAN NDAR $=40000$ \$END

$\$$ GUGDM NFLGDM(1)=1,0,0 IROOT=1 \$END

\$GUGDM2 WSTATE(1)=1.0,0.0,0.0 \$END

$\$ S T A T P T$ NSTEP $=200$ DXMAX $=0.01$ \$END

\$FORCE PURIFY=.T. \$END

\$DATA

optimized structure of the ground state

C1

$\begin{array}{lllll}\text { C } & 6.0 & -1.0167022851 & 1.0087744826 & 0.0000145687\end{array}$

$\begin{array}{lllll}\text { C } & 6.0 & -1.3674172585 & -0.3849472634 & 0.0000353282\end{array}$

$\begin{array}{lllll}\text { C } & 6.0 & -0.3596193747 & -1.4054233945 & -0.0000427400\end{array}$

$\begin{array}{llllll}\text { C } & & 6.0 & 1.0165240028 & -1.0086495934 & -0.0000053773\end{array}$

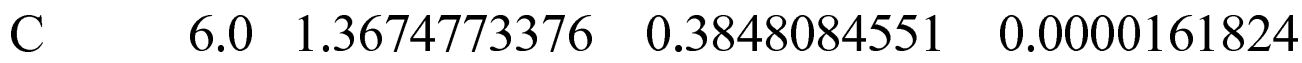

$\begin{array}{llllll}\mathrm{C} & 6.0 & 0.3597461553 & 1.4053957421 & -0.0000179471\end{array}$ 


$\begin{array}{lrrrr}\mathrm{H} & 1.0 & -1.7948572631 & 1.7482420459 & -0.0000885215 \\ \mathrm{H} & 1.0 & -2.4047272057 & -0.6620174181 & -0.0000030552 \\ \mathrm{H} & 1.0 & -0.6341882976 & -2.4426361212 & 0.0000172757 \\ \mathrm{H} & 1.0 & 1.7947973141 & -1.7479838929 & 0.0000983583 \\ \mathrm{H} & 1.0 & 2.4043930416 & 0.6618311430 & 0.0000763158 \\ \mathrm{H} & 1.0 & 0.6344802790 & 2.4430592282 & -0.0001005510 \\ \text { SEND } & & & & \\ \text { SVEC } & & & & \end{array}$

11 4.03968870E-01 1.11103080E-02-2.51509872E-05-1.80217429E-05 0.00000000E+00 1 2-2.18177499E-03 2.60369409E-04 1.88221699E-04 0.00000000E+00-9.81949399E-04 $13-1.01587600 \mathrm{E}-03-1.10029365 \mathrm{E}-03$ 6.65643918E-05 0.00000000E+00 0.00000000E+00 14 4.06748498E-01 1.11881925E-02 2.73038224E-06-3.01465715E-05 0.00000000E+00 1 5-2.20458963E-03-3.09090538E-05 3.17594703E-04 0.00000000E+00-1.06395210E-03 1 6-9.46704808E-04-1.10707271E-03-1.64208034E-05 0.00000000E+00 0.00000000E+00 17 4.09303551E-01 1.12595904E-02 2.70337661E-05-1.20715826E-05 0.00000000E+00 1 8-2.22150718E-03-2.92417746E-04 1.29874726E-04 0.00000000E+00-9.69564630E-04 1 9-1.05278428E-03-1.11340101E-03-5.06482016E-05 0.00000000E+00 0.00000000E+00 110 4.09503459E-01 1.12652900E-02 2.32356244E-05 1.80030313E-05 0.00000000E+00 1 11-2.23348321E-03-2.50713224E-04-1.91977766E-04 0.00000000E+00-9.93088238E-04 1 12-1.02856426E-03-1.11362686E-03 6.64702558E-05 0.00000000E+00 0.00000000E +00 113 4.05255122E-01 1.11463295E-02-4.45755523E-06 3.03931375E-05 0.00000000E+00 1 14-2.18689525E-03 4.45869145E-05-3.19313024E-04 0.00000000E+00-1.06116515E-03 1 15-9.43484393E-04-1.10359155E-03-1.52346557E-05 0.00000000E+00 0.00000000E+00 116 4.02893233E-01 1.10803447E-02-2.88042996E-05 1.21852280E-05 0.00000000E+00 1 17-2.17304686E-03 2.95171930E-04-1.25988440E-04 0.00000000E+00-9.55608142E-04 1 18-1.03788883E-03-1.09771877E-03-5.05872429E-05 0.00000000E+00 0.00000000E+00 1 19-1.13307708E-04 8.36497692E-04-1.14127091E-04 8.40412822E-04-1.14749171E-04 120 8.44772545E-04-1.14763401E-04 8.44513116E-04-1.13701932E-04 8.38573368E-04 1 21-1.13091047E-04 8.34542032E-04

21 5.74992090E-01 1.60803288E-02 1.25458503E-04 8.34458889E-05 0.00000000E+00 2 2-4.79885852E-03-2.78722001E-04-1.31587541E-04 0.00000000E+00-1.25413822E-03 2 3-1.28099469E-03-1.39303255E-03 2.96606205E-05 0.00000000E+00 0.00000000E+00 24 2.61447786E-01 7.31104445E-03 1.16420763E-04 8.21555497E-05 0.00000000E+00 2 5-2.18055654E-03-9.52979856E-04-2.46094840E-04 0.00000000E+00-6.08763062E-04 2 6-5.43226074E-04-6.33680904E-04 9.65066927E-05 0.00000000E+00 0.00000000E+00 2 7-3.13031325E-01-8.75555371E-03 1.22753443E-04 7.48994859E-05 0.00000000E+00 28 2.61470589E-03-5.14730639E-04-7.76161912E-04 0.00000000E+00 6.18121436E-04 $297.60684221 \mathrm{E}-04$ 7.57642708E-04-5.33970948E-05 0.00000000E+00 0.00000000E+00 2 10-5.73211781E-01-1.60325665E-02 1.26622442E-04 8.39770853E-05 0.00000000E+00 211 4.81261805E-03-2.88198672E-04-1.34764505E-04 0.00000000E+00 1.24743214E-03 212 1.27437964E-03 1.38717942E-03-2.83104418E-05 0.00000000E+00 0.00000000E+00 \$END 


\section{D.2 Energy calculations for ground state - benzene molecule}

$\$ C O N T R L$ SCFTYP=MCSCF RUNTYP=ENERGY EXETYP=RUN MAXIT $=100$ COORD $=$ UNIQUE

\section{\$END}

$\$$ MCSCF CISTEP $=$ GUGA MAXIT $=100$ \$END

\$SYSTEM MEMORY=40000000 TIMLIM=6000000.0 \$END

$\$ B A S I S$ GBASIS $=\mathrm{N} 31$ NGAUSS $=6$ NDFUNC $=1$ \$END

$\$$ GUESS GUESS $=$ MOREAD NORB $=102$ \$END

$\$$ DRT GROUP $=\mathrm{C} 1 \mathrm{FORS}=. \mathrm{T}$. $\mathrm{NMCC}=18 \mathrm{NDOC}=3 \mathrm{NVAL}=3$ \$END

\$GUGDIA NSTATE $=4$ ITERMX $=200$ \$END

\$LAGRAN NDAR $=40000$ \$END

$\$$ GUGDM NFLGDM(1)=0,1,0,0 IROOT $=2$ \$END

$\$$ GUGDM2 WSTATE(1) $=0.0,1.0,0.0,0.0$ \$END

$\$ S T A T P T$ NSTEP $=200$ DXMAX $=0.01 \$ E N D$

\$FORCE PURIFY=.T. \$END

\$DATA

optimized structure of the ground state

C1

\begin{tabular}{|c|c|c|c|}
\hline $\mathrm{C}$ & $6.0-1.0167022851$ & 1.0087744826 & 0.0000 \\
\hline $\mathrm{C}$ & $6.0-1.3674172585$ & -0.3849472634 & 0.0000353282 \\
\hline & $6.0-0.3596193747$ & -1.4054233945 & -0.0000427400 \\
\hline $\mathrm{C}$ & $6.0 \quad 1.0165240028$ & -1.0086495934 & -0.0000053773 \\
\hline & $6.0 \quad 1.3674773376$ & 0.3848084551 & 0.0000161824 \\
\hline C & $6.0 \quad 0.3597461553$ & 1.4053957421 & -0.0000179471 \\
\hline H & $1.0-1.7948572631$ & 1.7482420459 & -0.0000885215 \\
\hline $\mathrm{H}$ & $1.0-2.4047272057$ & -0.6620174181 & -0.0000030552 \\
\hline H & $1.0-0.6341882976$ & -2.4426361212 & 0.0000172757 \\
\hline & $1.0 \quad 1.7947973141$ & -1.7479838929 & 0.0000983583 \\
\hline & $1.0 \quad 2.4043930416$ & 0.6618311430 & 0.0000763158 \\
\hline & $1.0 \quad 0.6344802790$ & 2.4430592282 & -0.0001005510 \\
\hline
\end{tabular}




\section{\$VEC}

11 4.03968870E-01 1.11103080E-02-2.51509872E-05-1.80217429E-05 0.00000000E+00

1 2-2.18177499E-03 2.60369409E-04 1.88221699E-04 0.00000000E+00-9.81949399E-04

1 3-1.01587600E-03-1.10029365E-03 6.65643918E-05 0.00000000E+00 0.00000000E+00

14 4.06748498E-01 1.11881925E-02 2.73038224E-06-3.01465715E-05 0.00000000E+00

1 5-2.20458963E-03-3.09090538E-05 3.17594703E-04 0.00000000E+00-1.06395210E-03

1 6-9.46704808E-04-1.10707271E-03-1.64208034E-05 0.00000000E+00 0.00000000E+00

17 4.09303551E-01 1.12595904E-02 2.70337661E-05-1.20715826E-05 0.00000000E+00

1 8-2.22150718E-03-2.92417746E-04 1.29874726E-04 0.00000000E+00-9.69564630E-04

1 9-1.05278428E-03-1.11340101E-03-5.06482016E-05 0.00000000E+00 0.00000000E+00

110 4.09503459E-01 1.12652900E-02 2.32356244E-05 1.80030313E-05 0.00000000E+00

1 11-2.23348321E-03-2.50713224E-04-1.91977766E-04 0.00000000E+00-9.93088238E-04

1 12-1.02856426E-03-1.11362686E-03 6.64702558E-05 0.00000000E+00 0.00000000E+00

113 4.05255122E-01 1.11463295E-02-4.45755523E-06 3.03931375E-05 0.00000000E+00

1 14-2.18689525E-03 4.45869145E-05-3.19313024E-04 0.00000000E+00-1.06116515E-03

1 15-9.43484393E-04-1.10359155E-03-1.52346557E-05 0.00000000E+00 0.00000000E+00

116 4.02893233E-01 1.10803447E-02-2.88042996E-05 1.21852280E-05 0.00000000E+00

1 17-2.17304686E-03 2.95171930E-04-1.25988440E-04 0.00000000E+00-9.55608142E-04

1 18-1.03788883E-03-1.09771877E-03-5.05872429E-05 0.00000000E+00 0.00000000E+00

1 19-1.13307708E-04 8.36497692E-04-1.14127091E-04 8.40412822E-04-1.14749171E-04

120 8.44772545E-04-1.14763401E-04 8.44513116E-04-1.13701932E-04 8.38573368E-04

1 21-1.13091047E-04 8.34542032E-04

21 5.74992090E-01 1.60803288E-02 1.25458503E-04 8.34458889E-05 0.00000000E+00

2 2-4.79885852E-03-2.78722001E-04-1.31587541E-04 0.00000000E+00-1.25413822E-03

2 3-1.28099469E-03-1.39303255E-03 2.96606205E-05 0.00000000E+00 0.00000000E+00

24 2.61447786E-01 7.31104445E-03 1.16420763E-04 8.21555497E-05 0.00000000E+00

2 5-2.18055654E-03-9.52979856E-04-2.46094840E-04 0.00000000E+00-6.08763062E-04

2 6-5.43226074E-04-6.33680904E-04 9.65066927E-05 0.00000000E+00 0.00000000E+00

2 7-3.13031325E-01-8.75555371E-03 1.22753443E-04 7.48994859E-05 0.00000000E+00

28 2.61470589E-03-5.14730639E-04-7.76161912E-04 0.00000000E+00 6.18121436E-04

$297.60684221 \mathrm{E}-04$ 7.57642708E-04-5.33970948E-05 0.00000000E+00 0.00000000E+00

2 10-5.73211781E-01-1.60325665E-02 1.26622442E-04 8.39770853E-05 0.00000000E+00

211 4.81261805E-03-2.88198672E-04-1.34764505E-04 0.00000000E+00 1.24743214E-03

212 1.27437964E-03 1.38717942E-03-2.83104418E-05 0.00000000E+00 0.00000000E+00

2 13-2.55074083E-01-7.13457267E-03 1.16683444E-04 8.14806134E-05 0.00000000E+00

214 2.11994816E-03-9.55690194E-04-2.42412943E-04 0.00000000E+00 5.95515688E-04

215 5.30049823E-04 6.17530276E-04-9.67506467E-05 0.00000000E+00 0.00000000E+00

$2163.16715290 \mathrm{E}-01$ 8.85670567E-03 1.22159369E-04 7.46305349E-05 0.00000000E+00

2 17-2.63200769E-03-5.09143063E-04-7.72669506E-04 0.00000000E+00-6.27529726E-04

2 18-7.70061026E-04-7.67773223E-04 5.23454098E-05 0.00000000E+00 0.00000000E+00

2 19-1.52091059E-04 8.79886875E-04-6.89577822E-05 4.01367901E-04 8.26142119E-05

2 20-4.78256576E-04 1.51297950E-04-8.74713877E-04 6.74515965E-05-3.89783824E-04

2 21-8.36937879E-05 4.86028825E-04

\$END 


\section{D.3 Transitions dipole moment calculations for ground state}

\section{- benzene molecule}

$\$$ CONTRL SCFTYP=NONE CITYP $=$ GUGA MPLEVL $=0$ RUNTYP $=$ TRANSITN EXETYP $=$ RUN MAXIT $=100 \mathrm{COORD}=\mathrm{UNIQUE}$

\$END

$\$$ SYSTEM MEMORY $=40000000$ TIMLIM $=6000000.0$ \$END

$\$ B A S I S$ GBASIS $=\mathrm{N} 31$ NGAUSS $=6$ NDFUNC $=1$ \$END

\$GUGDIA NSTATE $=4$ ITERMX $=200$ \$END

$\$$ TRANST OPERAT $=$ DM numci $=2$ NFZC $=18$ IROOTS $(1)=1,2$ NOCC $=24$

\$END

$\$ D R T 1$ GROUP $=$ C1 FORS $=$. T. NFZC $=18$ NDOC $=3$ NVAL $=3$ \$END

$\$$ DRT2 GROUP $=\mathrm{C} 1$ FORS $=$.T. $\mathrm{NFZC}=18 \mathrm{NDOC}=3 \mathrm{NVAL}=3$ \$END

\$LAGRAN NDAR $=40000$ \$END

$\$$ GUGDM NFLGDM(1)=1,0,0 IROOT $=1$ \$END

\$GUGDM2 WSTATE(1)=1.0,0.0,0.0 \$END

$\$$ STATPT NSTEP $=200$ DXMAX $=0.01$ \$END

\$FORCE PURIFY=.T. \$END

\$DATA

optimized structure of the ground state

C1

$\begin{array}{lllll}\text { C } & 6.0 & -1.0167022851 & 1.0087744826 & 0.0000145687\end{array}$

$\begin{array}{lllll}\text { C } & 6.0 & -1.3674172585 & -0.3849472634 & 0.0000353282\end{array}$

$\begin{array}{lllll}\text { C } & 6.0 & -0.3596193747 & -1.4054233945 & -0.0000427400\end{array}$

$\begin{array}{llllll}\mathrm{C} & 6.0 & 1.0165240028 & -1.0086495934 & -0.0000053773\end{array}$

$\begin{array}{llllll}\mathrm{C} & 6.0 & 1.3674773376 & 0.3848084551 & 0.0000161824\end{array}$

$\begin{array}{llllll}\text { C } & 6.0 & 0.3597461553 & 1.4053957421 & -0.0000179471\end{array}$

$\mathrm{H} \quad \begin{array}{lllll}\mathrm{C} & 1.0 & -1.7948572631 & 1.7482420459 & -0.0000885215\end{array}$

$\mathrm{H} \quad \begin{array}{lllll}1.0 & -2.4047272057 & -0.6620174181 & -0.0000030552\end{array}$

$\begin{array}{lllll}\mathrm{H} & 1.0 & -0.6341882976 & -2.4426361212 & 0.0000172757\end{array}$

$\begin{array}{llllll}\mathrm{H} & & 1.0 & 1.7947973141 & -1.7479838929 & 0.0000983583\end{array}$

$\begin{array}{lllll}\mathrm{H} & 1.0 & 2.4043930416 & 0.6618311430 & 0.0000763158\end{array}$

$\begin{array}{llllll}\mathrm{H} & 1.0 & 0.6344802790 & 2.4430592282 & -0.0001005510\end{array}$

\$END 


\section{\$VEC1}

$113.86293936 \mathrm{E}-01$ 1.05061509E-02-7.17670682E-05 2.78097476E-05-1.22180709E-07

1 2-1.53261937E-03 6.58300040E-04-6.95530284E-05-6.02611692E-08-9.96528920E-04

1 3-9.27892691E-04-1.02641840E-03-7.55742585E-05 8.39844577E-09 5.57194196E-10

14 2.75880547E-01 7.45473795E-03-8.43140620E-05-2.39059829E-05-5.95892174E-08

1 5-5.18527289E-04 5.36933797E-04 3.71829107E-05 3.92407654E-09-6.96595552E-04

1 6-8.10475723E-04-7.68360082E-04 5.64911115E-05 1.44004209E-08-1.93950593E-08

17 4.65016666E-01 1.27065508E-02-9.86868271E-06-3.37800371E-05 1.31161213E-07

1 8-3.69158464E-03 5.59753487E-05 1.24039036E-05-4.73388995E-08-1.01199955E-03

1 9-9.62194961E-04-1.18178323E-03 1.13517588E-05 7.22212432E-09-1.48427278E-08

110 3.13125433E-01 8.49545289E-03 6.11076299E-05-4.71955774E-05 1.49843194E-07

1 11-1.39173472E-03-3.89262752E-04 6.61149994E-05 8.85623016E-08-8.02317825E-04

1 12-7.60414774E-04-8.44611155E-04-6.47719663E-05-4.79114207E-09-8.66811508E-09

113 2.87380494E-01 7.76124533E-03 9.53011667E-05-3.95433885E-06 7.08695773E-08

1 14-1.41407288E-04-8.11613404E-04 1.52294552E-04 2.44351309E-08-7.51790353E-04

1 15-8.56213568E-04-8.05419953E-04 7.95392162E-05 5.03088522E-09-3.36911390E-09

116 6.06938783E-01 1.66426612E-02 1.18664962E-05 7.00212835E-06-3.80982951E-08

1 17-5.36866793E-03-5.39770034E-05 2.71221010E-04-4.96866445E-08-1.28995449E-03

1 18-1.23159116E-03-1.50621283E-03 6.78754730E-06 8.87110862E-09-1.88384716E-08

1 19-1.17573946E-04 8.30930986E-04-9.51713833E-05 6.33938378E-04-1.23116781E-04

120 9.28141040E-04-9.97437354E-05 6.74090169E-04-9.66970241E-05 6.89341263E-04

1 21-1.63171957E-04 1.12239226E-03

2 1-2.31927390E-01-6.38618295E-03 5.18064693E-05 9.70033368E-05 5.73254813E-08

22 3.55823307E-04-1.10867137E-03-1.72447130E-04-4.68565268E-08 6.38642140E-04

23 5.05067196E-04 5.68967644E-04 3.07138271E-05-1.12552405E-08 6.87516300E-09

24 1.80267323E-01 4.91958132E-03-3.81791135E-05 1.20098003E-04 9.16963421E-08

25 4.26332510E-04 1.07004566E-03-8.33800648E-04 1.08998338E-07-5.07648796E-04

2 6-4.79534589E-04-4.73042644E-04 1.00311657E-04 2.79075110E-08-1.73185201E-08

27 6.81540450E-01 1.88812913E-02 6.47178787E-06 1.04030131E-04 2.31391712E-07

2 8-7.46326057E-03-1.07140589E-04-1.04498917E-03-5.57521442E-08-1.37272508E-03

2 9-1.32938861E-03-1.58373743E-03 4.93073379E-07 3.33047300E-09-1.82342904E-08

$2103.18375684 \mathrm{E}-01$ 8.73727555E-03 6.47857134E-05 8.72192609E-05 1.70121117E-07

2 11-7.66580470E-04-1.20007512E-03-1.37316437E-04 1.15630226E-07-8.51010426E-04

2 12-7.14199838E-04-7.98489584E-04-4.62174472E-05-1.43747054E-08-6.46992250E-09

2 13-1.00122359E-01-2.74588807E-03-2.13608203E-05 1.23170628E-04 9.45599264E-08

2 14-7.25275610E-04 9.52100043E-04-8.36183951E-04 4.76312287E-08 3.18000591E-04

215 2.66061649E-04 2.57640729E-04-8.93039260E-05-5.28602598E-09-1.33563641E-08

2 16-5.73437225E-01-1.59253459E-02 9.68422580E-06 1.14513803E-04 1.93955107E-08

217 6.68410369E-03-1.21507696E-04-1.06357737E-03 7.14541475E-08 1.12316006E-03

218 1.09746075E-03 1.30607043E-03 5.30547278E-06-3.14371509E-09 1.65293199E-08

219 5.36310292E-05-4.96058916E-04-4.33045696E-05 4.48418845E-04-1.91858753E-04

220 9.98502432E-04-7.97180919E-05 6.80034073E-04 1.87210799E-05-2.71329358E-04

221 1.60951995E-04-7.84270751E-04

\$END 
102 D.3. TRANSITIONS DIPOLE MOMENT CALCULATIONS FOR GROUND STATE 


\section{$\mathbf{E}$}

\section{APPENDIX: Non-adiabatic coupling}

\section{vector calculations}

In this thesis we have used the Columbus program for non-adiabatic coupling vector calculations of trans-stilbene and p-coumaric acid molecules.

The Columbus program is a set of Fortran programs for performing ab initio molecular electronic structure calculations. The programs can be used for calculations of electronic ground and excited states of atoms and molecules and is organized as a collection of separate programs which communicates between them via files. All of these programs required individual input files. In general, Columbus program can be used for geometry optimization and saddle point searches, automatic searches for minima on the crossing seam (canonical intersection), non-adiabatic coupling vector calculations, analytic gradient calculations etc. All of these calculations are done using the following methods

- Multiconfiguration Self-Consistent Field (MCSCF);

- Multireference Configuration Interaction with all single and double excitations (MRCISD);

- Multireference averaged coupled pair functional (MR-ACPF); 
- Multireference average quadratic coupled cluster (MR-AQCC);

In our case for calculating the non-adiabatic coupling vector we have considered the following steps, see for more details Ref, ${ }^{89,90}$

- geometry calculation via using SCF and MCSCF methods;

- molecular orbital file generated in MCSCF calculation;

- compute the CI wavefunctions;

- compute the corresponding symmetric one and two particle transition densities matrices and the antisymmetric one-particle density matrix and put all of these matrices on files;

- compute the Fock matrix;

- back transformation to the AO basis gives the final result for the total non-adiabatic coupling vectors. The non-adiabatic coupling vectors are given in cartesian components, and if it is necessary the coupling vector can be transformed to internal coordinates.

Forward are given the final results obtained using Columbus program for non-adiabatic coupling vectors for trans-stilbene and p-coumaric acid molecules. 


\section{E.1 Non-adiabatic couplings vectors for coumaric acid molecule}

$$
\begin{array}{ccc}
8.108307 \mathrm{E}-01 & 1.239252 \mathrm{E}-02 & 6.110511 \mathrm{E}-08 \\
-8.324037 \mathrm{E}-02 & 1.412484 \mathrm{E}-01 & -3.012922 \mathrm{E}-08 \\
2.442514 \mathrm{E}-01 & 1.336900 \mathrm{E}-01 & -2.163262 \mathrm{E}-08 \\
2.446028 \mathrm{E}-01 & -4.185924 \mathrm{E}-01 & -1.461293 \mathrm{E}-07 \\
3.243399 \mathrm{E}-01 & 6.622866 \mathrm{E}-02 & 2.845341 \mathrm{E}-07 \\
-6.679708 \mathrm{E}-01 & -3.027552 \mathrm{E}-01 & -1.433986 \mathrm{E}-07 \\
-1.375585 \mathrm{E}+00 & -2.519454 \mathrm{E}-01 & -7.598702 \mathrm{E}-08 \\
8.555618 \mathrm{E}-01 & 6.022155 \mathrm{E}-01 & 1.055739 \mathrm{E}-08 \\
-4.985751 \mathrm{E}-02 & 2.933025 \mathrm{E}-01 & -2.434362 \mathrm{E}-08 \\
-3.087958 \mathrm{E}-01 & 1.035637 \mathrm{E}-01 & 9.454610 \mathrm{E}-09 \\
-1.100732 \mathrm{E}-01 & -1.538594 \mathrm{E}-01 & -1.010323 \mathrm{E}-09 \\
5.349058 \mathrm{E}-03 & -1.375787 \mathrm{E}-01 & 5.032427 \mathrm{E}-08 \\
2.395797 \mathrm{E}-02 & -3.765749 \mathrm{E}-02 & -3.752482 \mathrm{E}-08 \\
1.417903 \mathrm{E}-02 & 1.006908 \mathrm{E}-02 & 1.887678 \mathrm{E}-08 \\
-9.798233 \mathrm{E}-04 & 3.276998 \mathrm{E}-02 & 1.897871 \mathrm{E}-08 \\
-1.610740 \mathrm{E}-02 & -3.736682 \mathrm{E}-02 & 1.102865 \mathrm{E}-08 \\
3.049188 \mathrm{E}-02 & 1.564262 \mathrm{E}-02 & -2.382618 \mathrm{E}-09 \\
1.162091 \mathrm{E}-02 & -3.501614 \mathrm{E}-02 & 4.038048 \mathrm{E}-09 \\
-4.134510 \mathrm{E}-03 & -3.220450 \mathrm{E}-02 & 6.377256 \mathrm{E}-09 \\
1.015883 \mathrm{E}-02 & -4.000011 \mathrm{E}-03 & 7.263315 \mathrm{E}-09
\end{array}
$$




\section{E.2 Non-adiabatic couplings vectors for stilbene molecule}

$\begin{array}{ccc}-1.076848 \mathrm{E}-01 & -2.596250 \mathrm{E}-01 & 6.582286 \mathrm{E}-09 \\ -1.075516 \mathrm{E}-01 & -2.596625 \mathrm{E}-01 & -1.773770 \mathrm{E}-08 \\ 1.706446 \mathrm{E}-01 & 7.612261 \mathrm{E}-02 & 1.966729 \mathrm{E}-08 \\ 1.705403 \mathrm{E}-01 & 7.649052 \mathrm{E}-02 & 6.459107 \mathrm{E}-09 \\ 3.375682 \mathrm{E}-03 & -8.602601 \mathrm{E}-02 & -2.872266 \mathrm{E}-09 \\ 3.166606 \mathrm{E}-03 & -8.626031 \mathrm{E}-02 & -5.672606 \mathrm{E}-09 \\ 9.444242 \mathrm{E}-02 & -8.603049 \mathrm{E}-02 & 1.050074 \mathrm{E}-08 \\ 9.440772 \mathrm{E}-02 & -8.590082 \mathrm{E}-02 & 1.306744 \mathrm{E}-08 \\ -1.148301 \mathrm{E}-01 & 7.538769 \mathrm{E}-02 & -4.157595 \mathrm{E}-09 \\ -1.148081 \mathrm{E}-01 & 7.498461 \mathrm{E}-02 & 2.087822 \mathrm{E}-09 \\ 7.915775 \mathrm{E}-02 & 4.506865 \mathrm{E}-02 & -2.570047 \mathrm{E}-08 \\ 7.934335 \mathrm{E}-02 & 4.530760 \mathrm{E}-02 & -9.602561 \mathrm{E}-09 \\ -1.528594 \mathrm{E}-01 & 1.356964 \mathrm{E}-01 & 2.927991 \mathrm{E}-09 \\ -1.527790 \mathrm{E}-01 & 1.355806 \mathrm{E}-01 & -1.106135 \mathrm{E}-08 \\ 2.045760 \mathrm{E}-02 & 1.057503 \mathrm{E}-01 & -7.017341 \mathrm{E}-09 \\ 2.045462 \mathrm{E}-02 & 1.057497 \mathrm{E}-01 & 7.690454 \mathrm{E}-09 \\ -1.163868 \mathrm{E}-02 & -2.219765 \mathrm{E}-03 & -5.077514 \mathrm{E}-09 \\ -1.164309 \mathrm{E}-02 & -2.230600 \mathrm{E}-03 & 5.894582 \mathrm{E}-09 \\ 1.278526 \mathrm{E}-02 & -1.381748 \mathrm{E}-02 & 2.751381 \mathrm{E}-09 \\ 1.277854 \mathrm{E}-02 & -1.380620 \mathrm{E}-02 & -6.396004 \mathrm{E}-09 \\ 3.162485 \mathrm{E}-02 & 3.496122 \mathrm{E}-03 & 3.125108 \mathrm{E}-09 \\ 3.162396 \mathrm{E}-02 & 3.495376 \mathrm{E}-03 & 3.380454 \mathrm{E}-09 \\ 9.108576 \mathrm{E}-03 & 9.668127 \mathrm{E}-03 & -5.155774 \mathrm{E}-10 \\ 9.111676 \mathrm{E}-03 & 9.678709 \mathrm{E}-03 & -1.770864 \mathrm{E}-09 \\ 3.876934 \mathrm{E}-03 & -1.056461 \mathrm{E}-02 & 8.337694 \mathrm{E}-09 \\ 3.880466 \mathrm{E}-03 & -1.057086 \mathrm{E}-02 & 5.109617 \mathrm{E}-09\end{array}$




\section{Acknowledgment}

I would like to thank to Prof. Dr. Jürgen Troe for giving me the opportunity to work in his departament at the Max-Planck Institut for Biophysical Chemistry, Göttingen .

I would like to thank to my direct supervisor Dr. A.A.Neufeld for giving me an interesting and chalenging $\mathrm{PhD}$ project and also his help and ideas have played an important role in this work. He constantly motivated me and guided me through the difficulties I encountered.

I would like to thank to Prof. Dr. Jörg Schroeder, Prof. Dr. Jürgen Troe , Prof. Dr. Peter Botschwina, Prof. Dr. Dirk Schwarzer, Prof. Dr. Helmut Grubmüller, Prof. Dr. Dietmar Stalke for accepting to be part of the committee thesis.

Finally, I would like to thank to my familly for their constant support and help. 



\section{Bibliography}

[1] E. E. Nikitin, Annu. Rev. Phys. Chem. 50, 1 (1999).

[2] H. Nakamura, Nonadiabatic transition, (2002)

[3] J. M. Anderson, Introduction to Quantum Chemistry, (1969)

[4] I. R. Levine, Quantum mechanics and molecular electronic structure, vol. I, (1970)

[5] L. Landau, Phys. Z. Sowjetunion 2, 46 (1932).

[6] C. Zener, Proc. R. Soc. London A 137, 696 (1932).

[7] Ping Ao, J. Rammer, Phys. Rev B 43, 5397 (1991).

[8] Y. Dong et al., Phys. Rev. A 74, 063810 (2006).

[9] M. Klintenberg, J. O. Thomas, Phys. Rev. B 56, 13006 (1997).

[10] W. R. Wadt, D. C. Cartwright, J. S. Cohen, Appl. Phys. Lett. 31, 672 (1977).

[11] S. H. Bauer, C. F. Aten, J. Chem. Phys. 39, 1253 (1963).

[12] W. Domcke, L. S. Cederbaum, Mol. Phys. 34, 1759 (1977).

[13] J. C. Tully, Faraday Discuss. 110, 407 (1998).

[14] N. L. Doltinis, Quantum simulations of complex Many-Body Systems: From Theory to Algorithm, Lectures Notes 10, 7917 (2000). 
[15] M. D. Hack, D. G. Truhlar J. Phys. Chem. A 104, 164111 (2005).

[16] U. Müller, G. Stock, J. Chem. Phys. 107, 825 (1997).

[17] D. Kohen, F. H. Stillinger, J. C. Tully, J. Chem. Phys. 109, 4713 (1998).

[18] K. Drukker, J. Comput. Phys. 153, 225 (1999).

[19] A. A. Neufeld, J. Chem. Phys. 119, 2488 (2003).

[20] A. A. Neufeld, D. Schwarzer, J. Schroeder, J. Troe, J. Chem. Phys. 119, 2502 (2003).

[21] A. A. Neufeld, J. Chem. Phys. 122, 164110 (2005).

[22] A. A. Neufeld, J. Chem. Phys. 122, 164111 (2005).

[23] J. Syage, W. Lambert, P. Felker, A. Zewail, R. Hochstrasser, Chem. Phys. 88, 266 (1982).

[24] J. Troe, Chem. Phys. Lett. 114, 241 (1984).

[25] D. H. Waldeck, Chem. Rev. 91, 415 (1991) and references therein.

[26] G. Orlandi, W. Siebrand, Chem. Phys. Lett. 30, 352 (1975).

[27] G. Orlandi, P. Palmieri, G. Poggi, J. Am. Chem. Soc. 101, 3492 (1979).

[28] P. M. Felker and A. H. Zewail, J. Phys. Chem. 89, 5402 (1985).

[29] F. Negri, G. Orlandi, J. Phys. Chem. 95, 748 (1991).

[30] A. W. Jasper, Chaoyuan Zhu, S. Nangia and D. G. Truhlar, Faraday Discuss, 127, 1 (2004).

[31] D. C. Tranca, A. A. Neufeld, J. Chem. Phys. 130, 141102 (2009).

[32] E. Wigner, J. Chem. Phys. 5, 720 (1937); E. Wigner, Trans. Faraday Soc. 34, 29 (1937). 
[33] H. Eyring, Trans. Faraday Soc. 34, 41 (1937); M. G. Evans, Trans. Faraday Soc. 34, 49 (1937).

[34] Y. Okuno, S. Mashiko, International Journal of Quantum Chemistry 102, 8 (2005).

[35] F. J. McLafferty, T. F. George, Chem. Phys. Lett. 37, 67 (1976).

[36] P. V. Parandekar, J. C. Tully, J. Chem. Theory Comput. 2, 229 (2006).

[37] X. Li, J. C. Tully, H. B. Schlegel, M. J. Frisch, J. Chem. Phys. 123, 084106 (2005).

[38] P. V. Parandekar, J. C. Tully, J. Chem. Phys. 122, 094102 (2005).

[39] M. J. Bedard et al., J. Chem. Phys. 123, 234106 (2005).

[40] G. Käb, J. Phys. Chem. A 110, 3197 (2006).

[41] J. C. Burant, J. C. Tully, J. Chem. Phys. 112, 6097 (2000).

[42] J. C. Tully, J. Chem. Phys. 93, 1061 (1990).

[43] J. C. Tully, J. Chem. Phys. 93, 1061 (1990).

[44] R. Kapral, Annu. Rev. Phys. Chem. 57, 129 (2006).

[45] P. A. Frantsuzov, Chem. Phys. Lett. 267, 427 (1997).

[46] W. T. Pollard and R. A. Friesner, J. Chem. Phys. 100, 5054 (1994).

[47] A.G. Redfield, IBM J.Res.Dev. 1,19 (1957) A.G. Redfield, Adv.Magn.Reson. 1,1, (1965)

[48] M. Dierlsen, S. Grimme, J. Chem. Phys. 122, 244101 (2005).

[49] R. Berger, C. Fischer, M. Klessinger, J. Phys. Chem. A 102, 7157 (1998).

[50] P. M. Johnson, H. Xu, J. Chem. Phys. 125, 164330 (2006). 
[51] M. P. Allen, D. J. Tildesley, Computer simulation of liquids, Oxford University Press, (1987).

[52] V. Molina, M. Merchan, B. O. Roos, J. Phys. Chem. A 101, 3478, (1997).

[53] B. B. Champagne, J. P. Pfansfiel, D. F. Plusquellic, D. W. Pratt, W. M. van Herpen, W. L. Meerts, J. Phys. Chem. 94, 6 (1990).

[54] Unpublished results of A.A. Neufeld.

[55] J.Stangl, V.Holy, G.Bauer, Rev. Mod. Phys. 76, 725 (2004).

[56] V.A.Shchukin, Dieter Bimerg, Rev. Mod. Phys. 71, 1125 (1999).

[57] K.Anderson, P.Malmqvist, B.O.Roos, A.J.Sadlej, K.Wolinski, J. Phys. Chem. 94, 5483 (1990).

[58] S.Yamanaka, M.Okumura, K.Yamaguchi, K.Hirao, Chem. Phys. Lett. 225, 213 (1994).

[59] M.Okumura, K.Yamaguchi, K.Awaga, Chem. Phys. Lett. 228, 575 (1994).

[60] K. Fuke, S. Sakamoto, M. Ueda, M. Itoh, Chem. Phys. Lett. 74, 546 (1980).

[61] W. L. Ryan, D. J. Gordon, D. H. Levy, J. Am. Chem. Soc. 124, 6194, (2002).

[62] H. Du, R. A. Fuh, J. Li, A. Corkan, J. S. Lindsey, Photochemistry and Photobiology, 68, 141 (1998); https://omlc.ogi.edu/spectra/PhotochemCAD/html

[63] H. Suzuki, Bulletin of Chemical Society of Japan 33, 944 (1960).

[64] J. Horwitz, E. H. Strickland, C. Billups, J. Amer. Chem. Soc. 92, 2119 (1970).

[65] J. Horwitz, E. H. Strickland, J. Biol. Chem. 246, 3749 (1971).

[66] Ian W. M. Smith, Chem. Soc. Rev. 37, 812 (2007). 
[67] J. I. Steinfeld, J. S. Francisco, W. L. Hase, Chemical Kinetics and Dynamics, Second Edition, Prentice-Hall Inc (1999).

[68] S. H. Courtney et. al, J. Chem. Phys. 89, 6697 (1988).

[69] A. Meyer, J. Schroeder and J. Troe, J. Phys. Chem. A 103, 10528 (1999).

[70] R. E. Weston Jr., J. R. Barker, J. Chem. Phys. A 110, 7888 (2006).

[71] A. Warshel, J. Chem. Phys. 62, 214 (1975).

[72] C. J. Finder, M. G. Newton, N. L. Allinger, Acta Cryst. B 30, 411 (1974).

[73] J. A. Bouwstra, A. Schouten, J. Kroon, Acta Cryst. C 40, 428 (1984).

[74] W.-G. Han, T. Lovell, T. Liu, L. Noodleman, Phys. Chem. Chem. Phys. 3, 167 (2002).

[75] M. Traetteberg, E. B. Frantsen, F. C. Mijlhoff, A. Hoekstra, J. Mol. Struct. 26, 57 (1975).

[76] S. P. Kwasniewski, L. Claes, J.-P. Francois, M. S. Deleuze, J. Chem. Phys. 118, 7823 (2003).

[77] L. H. Spangler, R. van Zee, T. S. Zwier, J. Phys. Chem. 91, 2782 (1987).

[78] A. A. Heikal, J. S. Baskin, L. Banares, A. H. Zewail, J. Phys. Chem. A 101, 572 (1997).

[79] J. S. Baskin, L. Banares, S. Pedersen, A. H. Zewail, J. Phys. Chem. 100, 11920 (1996).

[80] S. Nordholm, A. Baeck, Phys. Chem. Chem. Phys. 3, 2289 (2001).

[81] J. Qian, S. L. Schultz, J. M. Jean, Chem. Phys. Lett. 233, 9 (1995).

[82] P. M. Felker, W. R. Lambert, A. H. Zewail, J. Chem. Phys. 82, 3003 (1985).

[83] L. Banares, A. A. Heikal, A. H. Zewail, J. Phys. Chem. 96, 4127 (1992).

[84] T. Elsaesser, W. Kaiser, Annu. Rev. Phys. Chem. 42, 83 (1991). 
[85] J. W. Perry, N. F. Scherer, A. H. Zewail, Chem. Phys. Lett. 103, 1 (1983).

[86] T. Gensch, C. C. Gradinaru, I. H. M. van Stokkum, J. Hendriks, K. J. Hellingwerf, R. van Grondelle, Chem. Phys. Lett. 356, 347 (2002).

[87] N. Mataga, H. Chosrowjan, S. Taniguchi, J. Photochem. Photobiol. C 5, 155 (2004).

[88] K. Heyne, O. F. Mohammed, A. Usman, J. Dreyer, E. T. J. Nibbering, M. A. Cusanovich, J. Am. Chem. Soc. 127, 18100 (2005).

[89] Hans Lischka, Michal Dallos, Peter G. Szalay, David R. Yarkony, Ron Shepard, J. Chem. Phys. 120, 7322 (2004).

[90] Hans Lischka, Michal Dallos, Peter G. Szalay, David R. Yarkony, Ron Shepard, J. Chem. Phys. 120, 7330 (2004). 


\section{LEBENSLAUF}

$\begin{array}{ll}\text { Familienname: } & \text { Tranca } \\ \text { Vornamen: } & \text { Diana-Constanta } \\ \text { Geburtsdatum: } & 26.05 .1981 \\ \text { Geburtsort: } & \text { Craiova, Rumänien } \\ \text { Staatsburgerschaft: } & \text { Rumänisch } \\ \text { Familienstand: } & \text { ledig } \\ \text { E-Mail: } & \text { diana.tranca@tu-harburg.de }\end{array}$

01.04.09 bis jetzt

$01.10 .08-31.03 .09$

September 2005 - März 2009

$2004-2005$

$2000-2004$

$1996-2000$

$1988-1996$

\section{Stipendien und Auszeichnungen}

$15.09 .05-31.09 .08$

$01.12 .04-31.07 .05$

$1998-1999$

\section{Lehrerfahrung}

$15.04 .08-31.03 .09$
Wissenschaftliche Mitarbeiterin an der TU Hamburg-Harburg

Wissenschaftliche Mitarbeiterin an der Universităt Göttingen, Fakultẳ für Physik

Doktorandin am Max-Planck-Institut für biophysikalische Chemie, Gruppe: Dr. Anatole Neufeld

Titel der Dissertation: Quantum-classical modeling of nonadiabatic transitions in polyatomic systems

Masterarbeit in Zusammenarbeit mit der FU Berlin Titel der Masterarbeit: Thermodynamik und Tunneln in kleinen magnetischen Systemen

Studium der Physik und Informatik an der Universität von Craiova, Rumänien

Diplom in Physik und Informatik (Juni 2004)

Titel der Diplomarbeit: Client/Server-Datenbanken

Abitur, "Carol I"-Gymnasium, Craiova, Rumånien

Grundschule

DFG (Deutsche Forschungsgemeinschaft);

Stipendium am MPI für biophysikalische Chemie, Göttingen

Socrates/Erasmus-Stipendium an der FU Berlin

Zweiter Platz in der Geschichtsolympiade

Tutorin in Mathematik an der Universităt Göttingen, Fakultăt für Physik, in den folgenden Kursen: Mathematische Methoden in Physik I, Mathematische Methoden in Physik II, Repetitorium für mathematische Physik I

in Zusammenarbeit mit dem Gymnasien "Odobleja" und "Elena Cruza" 


\section{Veröffentlichungen}

Quantum-classical modeling of photoisomerization of polyatomic molecules, D.C.Tranca, A.A. Neufeld, accepted to J.Chem.Phys.

On the structure of hot absorption spectra of polyatomic molecules: Solvent effect on the transition energy gap, D.C. Tranca, A.A. Neufeld, J. Chem. Phys. 130 (2009), 141102

\section{Konferenzen und Tutorials}

$26.05 .05-28.05 .05$

$21.09 .06-29.09 .06$

03.03.08- 17.03.08

$09.09 .08-11.09 .08$

$31.08 .09-04.09 .09$

$90^{\text {th }}$ International Bunsen Discussion Meeting Time resolved transformations in complex molecular environments: pushing the frontiers in experiment and theory, Max-Planck-Institut für biophysikalische Chemie

CECAM (Centre European de Calcul Atomique et Moleculaire) Tutorial: General Introduction to Simulation Methods, Lyon, Frankreich

IFF Spring School 2008, Jülich

$1^{\text {s }} \mathrm{PhD} /$ Postdoc Konferenz des MPI für biophysikalische Chemie, Eisenach

Dynamics Days Europe Konferenz, Göttingen

\section{IT-Kenntnisse}

Betriebssysteme:

Microsoft Windows 95/98/2000, Unix, Linux

Programmiersprachen:

C, C++, Grundkenntnisse in Fortran 90, Maple, Mathcad, Matlab

Datenbanken:

Microsoft Access, Fox Pro 2.6, Informix, SQL Server 2000, Visual Basic

Office:

Latex, Open Office, Microsoft Word, Excel, Power Point

Zeichenprogramme:

Paint Shop Pro 7.02, Adobe Photoshop 6.0

Web-Technologie:

HTML

Sprachen

Rumänisch (Muttersprache)

Englisch - gute Kenntnisse

Französisch - mittlere Kenntnisse

Deutsch - mittlere Kenntnisse 